San Jose State University

SJSU ScholarWorks

Master's Theses

Master's Theses and Graduate Research

1992

\title{
Effect of oxidation on magnetic and structural properties of terbium containing magneto-optical thin films
}

Ashok K. Gupta

San Jose State University

Follow this and additional works at: https://scholarworks.sjsu.edu/etd_theses

\section{Recommended Citation}

Gupta, Ashok K., "Effect of oxidation on magnetic and structural properties of terbium containing magneto-optical thin films" (1992). Master's Theses. 464.

DOI: https://doi.org/10.31979/etd.auxp-6dja

https://scholarworks.sjsu.edu/etd_theses/464

This Thesis is brought to you for free and open access by the Master's Theses and Graduate Research at SJSU ScholarWorks. It has been accepted for inclusion in Master's Theses by an authorized administrator of SJSU ScholarWorks. For more information, please contact scholarworks@sjsu.edu. 


\section{INFORMATION TO USERS}

This manuscript has been reproduced from the microfilm master. UMI films the text directly from the original or copy submitted. Thus, some thesis and dissertation copies are in typewriter face, while others may be from any type of computer printer.

The quality of this reproduction is dependent upon the quality of the copy submitted. Broken or indistinct print, colored or poor quality illustrations and photographs, print bleedthrough, substandard margins, and improper alignment can adversely affect reproduction.

In the unlikely event that the author did not send UMI a complete manuscript and there are missing pages, these will be noted. Also, if unauthorized copyright material had to be removed, a note will indicate the deletion.

Oversize materials (e.g., maps, drawings, charts) are reproduced by sectioning the original, beginning at the upper left-hand corner and continuing from left to right in equal sections with small overlaps. Each original is also photographed in one exposure and is included in reduced form at the back of the book.

Photographs included in the original manuscript have been reproduced xerographically in this copy. Higher quality $6 " \mathrm{n}$ × 9 " black and white photographic prints are available for any photographs or illustrations appearing in this copy for an additional charge. Contact UMI directly to order.

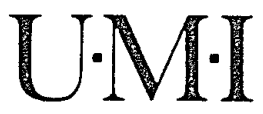

University Microfilms Internatıonal

A Bell \& Howell information Company

300 North Zeeb Road. Ann Arbor. MI 48106-1346 USA

313:761-4700 800:521-0600 
-... . . . 
Order Number 1351035

Effect of oxidation on magnetic and structural properties of terbium containing magneto-optical thin films

\author{
Gupta, Ashok K., M.S.
}

San Jose State University, 1992

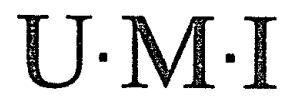

300 N. Zeeb Rd.

Ann Arbor, MI 48106 

EFFECT OF OXIDATION ON MAGNETIC AND BTRUCTURAL PRORERTIEB OF TERBIUM CONTAINING MAGNETO-OPTICAL THIN FILMS

\author{
A Thesis \\ Presented to \\ The Faculty of the Department of Materials Engineering \\ San Jose State University
}

In Partial Fulfillment

of the Requirements for the Degree

Master of Science

By

Ashok K. Gupta

December, 1992 
APPROVED FOR THE DEPARTMENT OF MATERIALS ENGINEERING

Gina Selvaduray

Dr. Gun Selvaduray

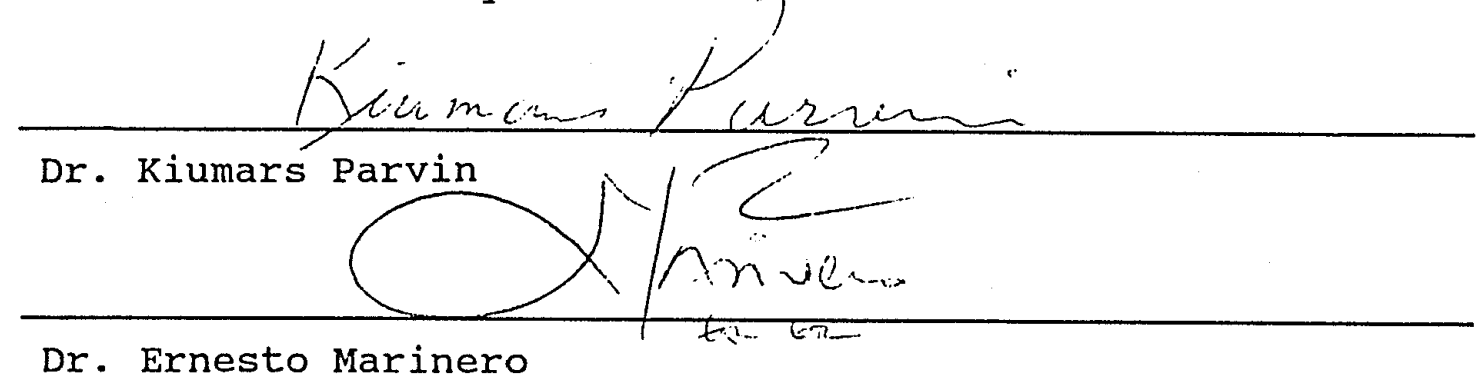

APPROVED FOR THE UNIVERSITY

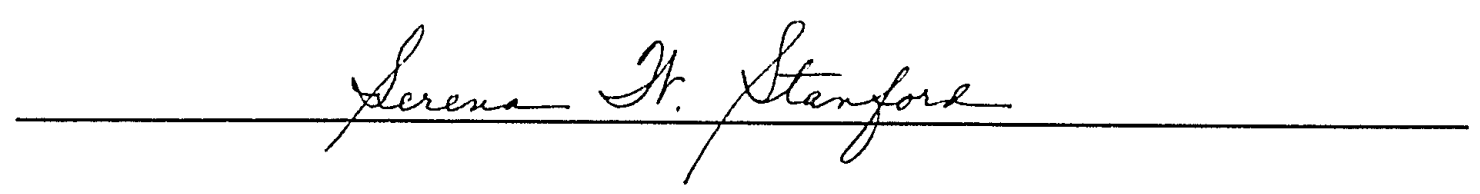




\begin{abstract}
EFFECT OF OXIDATION ON MAGNETIC AND STRUCTURAL PROPERTIES

OF TERBIUM CONTAINING MAGNETO-OPTICAL THIN FILMS
\end{abstract}

By Ashok K. Gupta

The changes in magnetic and magneto-optical behavior of bare $\mathrm{TbFe}$ and TbFeCo thin films due to oxidation at $70^{\circ} \mathrm{C}$ were investigated. The decrease in Kerr rotation and coercivity due to oxidation is explained on the basis of compositional and structural changes observed using ESCA and TEM. In these films, preferential oxidation of $\mathrm{Tb}$ was found to occur. This leads to metallic Fe and Co dispersed inside an oxide layer of Tb. This layer, with a complex structure, grows parabolically with oxidation time, indicating the oxidation process to be diffusion dependent. The presence and growth of this layer modifies the magnetic and magneto-optical properties of these alloy films. The addition of co improves Kerr rotation and resistance to oxidation-induced changes in these films. 
I am grateful to Professor G. Selvaduray and Professor K. Parvin, my thesis advisors from San Jose State University, for their guidance, support, and helpful advice during the course of this work. I am also thankful to the following at IBM Corporation: Dr. E. Marinero for sponsoring this work and providing indispensable support and guidance; Dr. $\mathrm{H}$. Notarys and Dr. D. Miller for their support in film preparation and ESCA analysis; and Dr. F. Sequeda for his advice on various topics. Finally, I am thankful to Dr. D. Chung for his help with TEM work. 


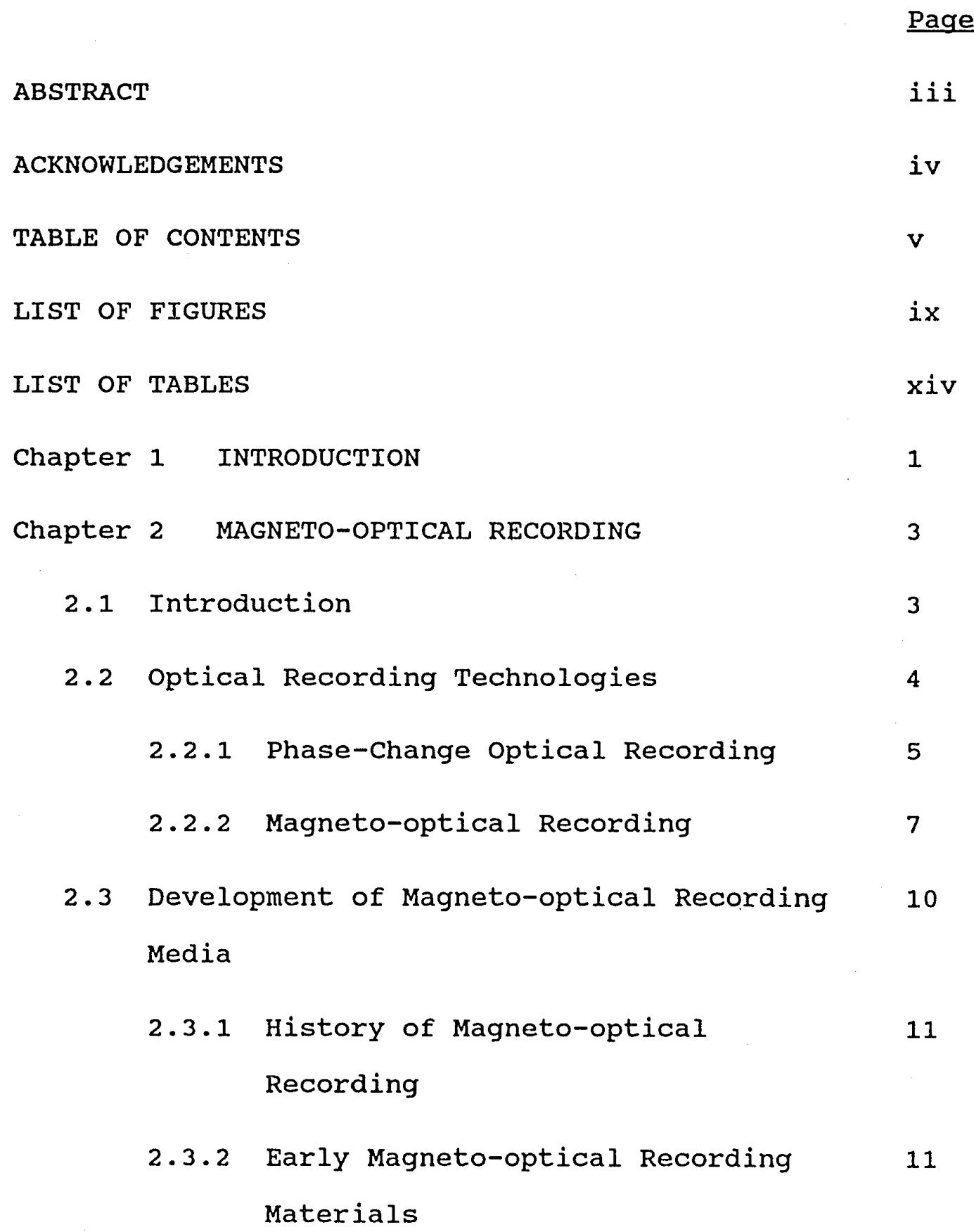


2.3.3 Recent Magneto-optical Recording 12 Materials

2.3.4 Future Expectations 16

2.4 Desired Characteristics of MO Media 16

2.4.1 High Signal-to-Noise Ratio 18

2.4.2 High Recording Density or High Bit 19 Density

2.4.3 Low Error Rate and High Data 21 Transfer Rate

2.4 .4 Overwrite Capability 22

2.4.5 Media Cyclability 23

2.4.6 Data Stability 23

2.5 Properties of Thin RE-TM Alloy Films 24

2.5.1 Film Microstructure 24

2.5.2 Magnetization versus Temperature 26 and Concentration of $\mathrm{RE}$ Element in the Film

2.5.3 Effect of Temperature and RE 30 Concentration on coercivity 
2.5.4 Effect of Temperature and Film Composition on Kerr Rotation

2.5.5 Magnetic Anisotropy

2.6 Write, Read, and Erasure Processes for Magneto-optical Recording Media

2.6.1 The Writing Process

2.6.2 The Reading Process

2.6.3 The Erasure Process

2.7 Aging and Chemical stability of RE-TM Films

2.7.1 Oxidation Behavior of RE-TM Media

2.7.2 Oxidation Protection for MO Media 46

Chapter 3 EXPERIMENTAL PROCEDURE

3.1 Experimental Plan

3.2 Materials

3.2.1 Targets

3.2.2 Substrates

3.3 Sample Preparation Procedure 
3.4 Testing and Film Characterization Methods 57

3.4.1 Kerr Tester 57

3.4.2 Vibrating Sample Magnetometer (VSM) 59

3.4.3 Surface Analysis by X-ray 60

Photoelectron Spectroscopy (XPS)

3.4.4 Transmission Electron Microscopy 64 (TEM)

$\begin{array}{lll}\text { Chapter } 4 & \text { RESULTS AND DISCUSSION } & 69\end{array}$

4.1 Effect of Oxidation on Kerr Rotation and 69 Coercivity

4.2 Magnetic Measurements using VSM 76

4.3 Oxidation Depth versus Annealing Time 82

4.4 Changes in Film structure with oxidation 95

4.5 Discussion of Results 99

$\begin{array}{lll}\text { Chapter } 5 \text { CONCLUSIONS } & 113\end{array}$

$\begin{array}{lll}\text { Chapter } 6 & \text { RECOMMENDATIONS FOR FUTURE WORK } & 115\end{array}$

$\begin{array}{lll}\text { Chapter } 7 & \text { REFERENCES } & 116\end{array}$ 


\section{IIST OF FIGURES}

\section{Figure}

1 Principle of phase-change recording. 6

2 Thermal effects in phase-change materials 8 based on thermal history.

3 Principle of Mo recording. (a) Rotation

of a plane polarized beam upon reflection from an Mo media, (b) Kerr rotation angle $\left(\theta_{k}\right)$.

6

8

9

Schematic of a cylindrical domain in an Mo thin film.

5 Temperature dependence of magnetization and coercivity for an RE-TM alloy.

compensation temperature through change in net magnetization.

$7 \quad$ Coercivity versus temperature for $\mathrm{Tb}_{\mathrm{x}} \mathrm{Fe}_{\mathrm{y}}$ films.

8 Effect of RE concentration on compensation and Curie temperature for RE-Fe films. (a) Gd, (b) Tb, (c) Dy. Effect of Curie temperature on Kerr rotation of various RE-TM alloys (d).

9 Writing, reading, and erasing processes for Mo recording. TbFe films.

11 The changes in composition of TbFe films with depth, due to oxidation.

during oxidation of a TbFe film at $200^{\circ} \mathrm{C}$ for 5 hours. 
13 Parabolic growth rate oxidation process for a TbFe alloy film heated to $200^{\circ} \mathrm{C}$ for 5 hours.

14 Effect of various alloying elements on corrosion and pinhole formation, as measured by film reflectance.

15 Schematics of the Kerr tester used for Kerr rotation and coercivity measurements.

16 Block diagram of the vibrating sample magnetometer.

17 Energy level diagram showing the electron transition during the ESCA technique.

18 Electron optical column in a conventional transmission electron microscope.

19 (a) Formation of focused diffraction pattern in the back focal plane of the objective lens, (b) position of aperture to form a BF image, and (c) tilted illumination to form a CDF image.

20 Changes in the film incident Kerr hysteresis loop of TbFe films with annealing at $70^{\circ} \mathrm{C}$.

21 Changes in the film incident Kerr hysteresis loop of TbFeCo films with annealing at $70^{\circ} \mathrm{C}$.

22 Changes in the substrate incident Kerr hysteresis loop of TbFeCo films with annealing at $70^{\circ} \mathrm{C}$.

23 Normalized Kerr rotation $\left(\theta_{k}\right)$ and coercivity $\left(H_{c}\right)$ of TbFe films as a function of annealing time at $70^{\circ} \mathrm{C}$. 
24 Normalized Kerr rotation $\left(\theta_{k}\right)$ and coercivity $\left(H_{c}\right)$ of TbFeco films as a function of annealing time at $70^{\circ} \mathrm{C}$.

25 (a) Growth in the parallel component of magnetization of TbFe films with annealing at $70^{\circ} \mathrm{C}$.

(b) Changes in the perpendicular hysteresis loop of TbFe films with annealing at $70^{\circ} \mathrm{C}$.

26 Changes in the parallel and normal components of magnetization of $\mathrm{TbFeCo}$ films with annealing at $70^{\circ} \mathrm{C}$.

27 (a) Oxidation-induced changes in TbFeco films annealed at $70^{\circ} \mathrm{C}$, as a function of time $e^{1 / 2}$.

(b) Oxidation-induced changes in TbFe films annealed at $70^{\circ} \mathrm{C}$, as a function of time $e^{1 / 2}$.

28 Effect of the 7059 glass substrate on the slope of VSM measurements.

29 Composition of TbFeCo films, by ESCA depth profiling. (a) As-deposited, (b) annealed for 92 hours at $70^{\circ} \mathrm{C}$.

30 ESCA depth profiles showing compositional changes for TbFe films with annealing.

31 Peak shifts in the binding energy of $\mathrm{Fe}$ and co due to oxidation.

32-1 Surface energy spectra of annealed TbFe films from ESCA.

32-2 Binding energy spectra of annealed TbFe films from ESCA for $\mathrm{Tb}-4 \mathrm{~d}$.

32-3 Binding energy spectra of annealed TbFe films from ESCA for $\mathrm{Fe}-2 \mathrm{p}$. 
32-4 Binding energy spectra of annealed TbFe films from ESCA for $0-1$ s. depth profiling; of TbFeco films annealed at $200^{\circ} \mathrm{C}$ for 336 hours.

33-2 Binding energy spectra of TbFeco films annealed at $200^{\circ} \mathrm{C}$ for 336 hours.

34 Diffraction pattern and BF images of asdeposited TbFe $(a, b)$, and TbFeCo $(c, d)$ films; with SiN overcoat.

35-1 (a) Diffraction pattern, (b) BF image, and (c) CDF image; of oxidized TbFeCo films with SiN overcoat (thin area).

35-2 (a) Diffraction pattern, (b) BF image, and (c) CDF image; of oxidized TbFe films with SiN overcoat (thin area).

36 Oxidation depth as a function of time ${ }^{1 / 2}$, for $\mathrm{TbFe}$ and TbFeCo films annealed at $70^{\circ} \mathrm{C}$.

37 Schematic of compositional changes with oxidation of TbFe and TbFeCo films.

Kerr loops for (40 nm siN/ xFe/ $100 \mathrm{~nm}$ FeTb/ $40 \mathrm{~nm} \mathrm{SiN/} \mathrm{substrate)} \mathrm{films} \mathrm{for} \mathrm{x}=$; (a) $2.8 \mathrm{~nm}$, (b) $9.4 \mathrm{~nm}$, and (c) $15.1 \mathrm{~nm}$.

38-2 VSM hysteresis loops for (40 nm SiN/ $\mathrm{xFe} /$ $100 \mathrm{~nm}$ FeTb/ $40 \mathrm{~nm}$ SiN/ substrate) films for $x=$; (a) $2.8 \mathrm{~nm}$, (b) $9.4 \mathrm{~nm}$, (c) 15.1 $\mathrm{nm}$.

39 Computed Kerr rotation and ellipticity originating from a two-layer structure consisting of an Mo layer overcoated with an absorbing but nonmagneto-optically active layer. 
40 Kerr loop of a TbFeco film, annealed for 72 hours at $70^{\circ} \mathrm{C}$, with compensator placed in optical setup.

41 (a) Normalized reflectivity of TbFe films annealed at $70^{\circ} \mathrm{C}$ as a function of $t i m e^{1 / 2}$. (b) Normalized reflectivity of TbFeCo films annealed at $70^{\circ} \mathrm{C}$ as a function of time ${ }^{1 / 2}$. 


\section{LIST OF TABLES}

Table $\quad$ Page

1 Magnetic properties of various amorphous

14 rare earth-transition metal alloys.

2 Magnetic and magneto-optical properties of various rare earth ( $R E)$-transition metal (TM) alloys.

Properties of interest for MO recording media. crystallization. 


\section{Chapter 1}

\section{INTRODUCTION}

A great deal of work has been done in the area of magnetooptical (MO) materials during the last decade in order to develop data storage applications. $(1,2,3,4)$ These materials are currently utilized in rewritable magneto-optical recording technology. This technology offers various advantages such as erasability, rewritability, and random access capability, as in the case of electromagnetic recording. It also has the additional advantages of removable media and high recording densities.

Amorphous films of rare earth-transition metal (RE-TM) alloys, such as TbFe and GdCo and their variants, are among the most suitable materials for the magneto-optical recording. Some of the required properties of these materials are their high coercivity at room temperature, high perpendicular anisotropy, and a high Kerr rotation. However, the rare earth element component of these materials is very reactive. Thus, unprotected thin films of the rare earthtransition metal alloys are very susceptible to degradation due to oxidation. Therefore, it is of the utmost importance to study the stability behavior of these films.

This investigation was undertaken to study the changes in the film characteristics due to oxidation of two of these 
alloy systems, namely TbFe and TbFeCo. The oxidation-induced compositional and structural changes were determined using electron spectroscopy for chemical analysis (ESCA) and transmission electron microscopy (TEM). The changes in magnetic and magneto-optical properties due to oxidation were measured using a vibrating sample magnetometer (VSM) and a Kerr tester, respectively. The changes in compositional and structural properties upon oxidation were related to the corresponding changes in magneto-optical behavior of these films. Furthermore, the changes in these properties of TbFe films were compared to the properties of TbFeco films.

The history and principles of various optical recording techniques are discussed in chapter 2 of this thesis. The requirements for magneto-optical recording technology, the properties of magneto-optical materials, and the work done by the other investigators in order to determine the stability characteristics of these materials are also discussed in the same chapter. The experimental methods and techniques employed for this study are described in chapter 3. This is followed by a discussion of the results in chapter 4 . The main findings are presented in Chapter 5. Chapter 6 contains some recommendations for possible future work to be done in this area in order to further enhance the understanding of these materials. 
Chapter 2

\section{MAGNETO-OPTICAL RECORDING}

\subsection{Introduction}

During the last few years the developments in optical recording materials and high power semiconductor diode lasers have brought the optical recording technology into the realm of reality. Compact disks (CD), video and digital audio, are among the well known products that use optical recording technology. These products are readily available in the marketplace. For the 'read only' media used in the above products, the information signal is recorded on the disk in the form of micron sized pits. The modulation of a laser beam, reflected by the pits, is employed in the playback process. The desired video or audio signal is obtained by electronic signal processing. $(5,6)$

Write-once drives, (1) with over $2 \mathrm{~Gb}$ of storage per disk drive, offer the advantages of high areal density $\left(\sim 10^{8}\right.$ bits $/ \mathrm{cm}^{2}$ ), high data transfer rates (about $5 \mathrm{Mb} / \mathrm{s}$ ), random access capability (about $100 \mathrm{~ms}$ average access time), large head-to-disk separation (of the order of $1 \mathrm{~mm}$ ), and removability of the media. Head-to-disk separation in optical recording offers the additional advantage of eliminating one of the major problems in magnetic storage 
technology, namely, head crashes.

Thus, optical storage can be expected to play a key role in the storage product hierarchy, where high recording densities and removable media are desired. For optical recording to become even more attractive, it must add overwrite capability to the above list of advantages. Overwrite refers to the ability to locally reverse information-carrying marks from one state to another as a one step process. Current products need an additional disk revolution to erase written tracks.

\subsection{Optical Recording Technologies}

Some of the techniques currently being studied for reversible optical storage are based on magneto-optical phenomena, amorphous-crystalline phase transitions, thermomechanical deformation of dye-loaded polymers, persistent photochemical and photophysical spectral hole burning, and holographic techniques. (5) spectral hole burning and holographic recording technologies are still in the exploratory research level. Magneto-optical, phase-change, and dye-loaded polymer materials have been demonstrated for rewritability in practical device applications. The dyeloaded polymer is a low-end technology and will not be discussed here. The principles of optical recording for phase-change media and magneto-optical media are described 
below.

\subsubsection{Phase-Change optical Recording $(1,3,4)$}

Figure 1 shows the principle of phase-change recording. To begin with, the recording media is assumed to be in a crystalline state. A focused laser beam (less than 100ns pulse width) is used to increase the temperature of a small region of the material above the melting point. Subsequently, the laser power is rapidly decreased, subjecting the above melted region to thermal quenching. This process changes the microstructure of the region from a crystaliine state to an amorphous state. This recorded amorphous domain corresponds to the "writing step."

The difference in reflectivity between the crystaline and amorphous phases of the media is used for the "readout" operation. A low powered continuous laser beam is used to perform this operation. The difference in reflectivity between the two phases may be enhanced by using optical interference effects, e.g., by tuning the layer media thickness.

The erasure process involves annealing the amorphous region. This is accomplished by using a low power laser pulse for a longer duration. The objective is to raise the temperature of the region between the glass transition temperature, $T_{g}$, and melting point, $T_{m}$ until crystalline 


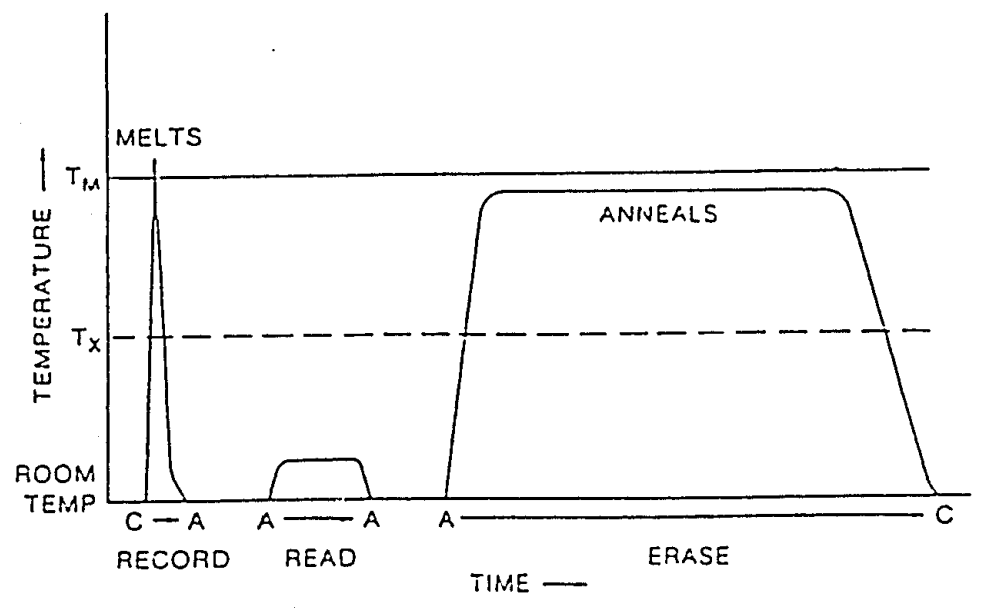

Figure 1. Principle of phase-change recording. (4) 
regrowth has fully occurred. Figure $2^{(3)}$ indicates various thermal effects occurring in a phase-change material, depending upon its thermal history. Most of the phase-change media reported are based on Se-Te-Sn, Se-In-Sb, TeO $: G e, S n$ families. (1)

\subsubsection{Magneto-optical (MO) Recording}

The magneto-optical recording process is based on the principle of the Polar Kerr effect (Faraday effect) $(3,7,8,9)$ The Kerr effect occurs when a linearly polarized beam of light is incident on a magnetized material, with the direction of magnetization parallel to the direction of the incident beam. Upon reflection, the beam becomes elliptically polarized as shown in Figure 3. This elliptical polarization of a plane polarized incident beam is known as the Kerr effect. The corresponding effect in transmission is known as the Faraday effect. The plane of polarization of the reflected beam rotates clockwise or counterclockwise, depending upon whether the direction of magnetization in the film is parallel or antiparallel to the direction of the incident beam. The rotation angle depends on the extent of magnetization in the direction parallel to the incident beam direction and the details of the optical interaction within the MO material. It is independent of the strength of the applied external field. The coefficients of reflection may 


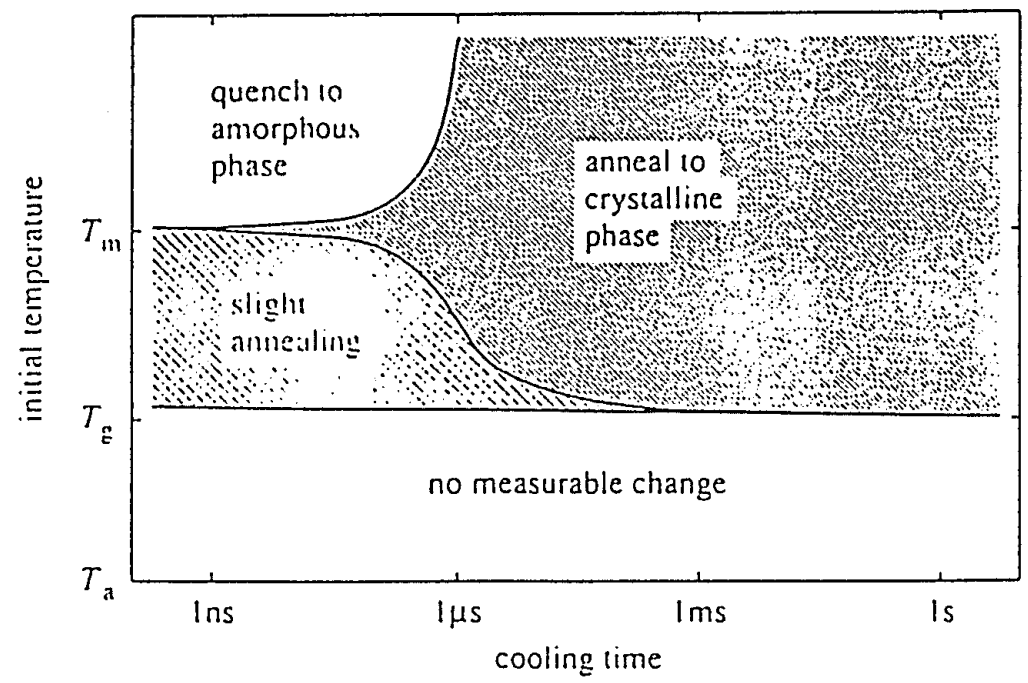

Figure 2. Thermal effects in phase-change materials based on thermal history. (3) 


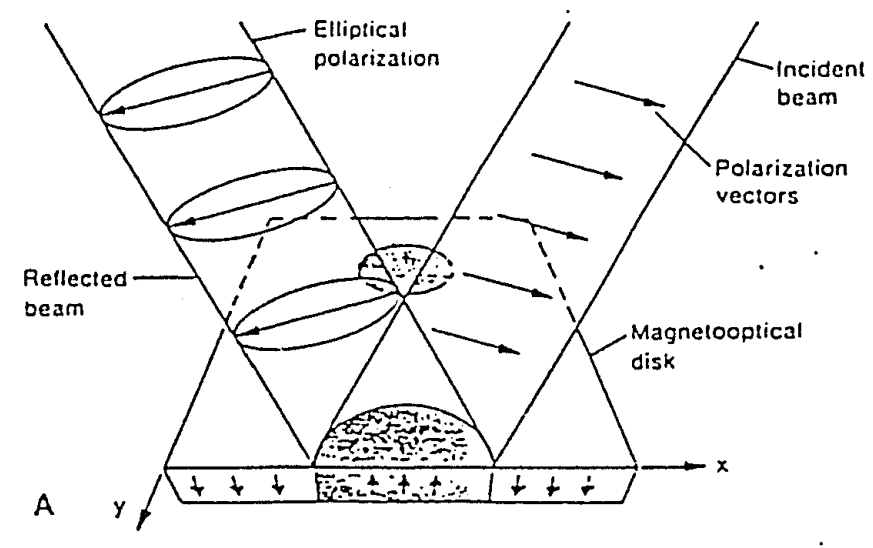

(a)

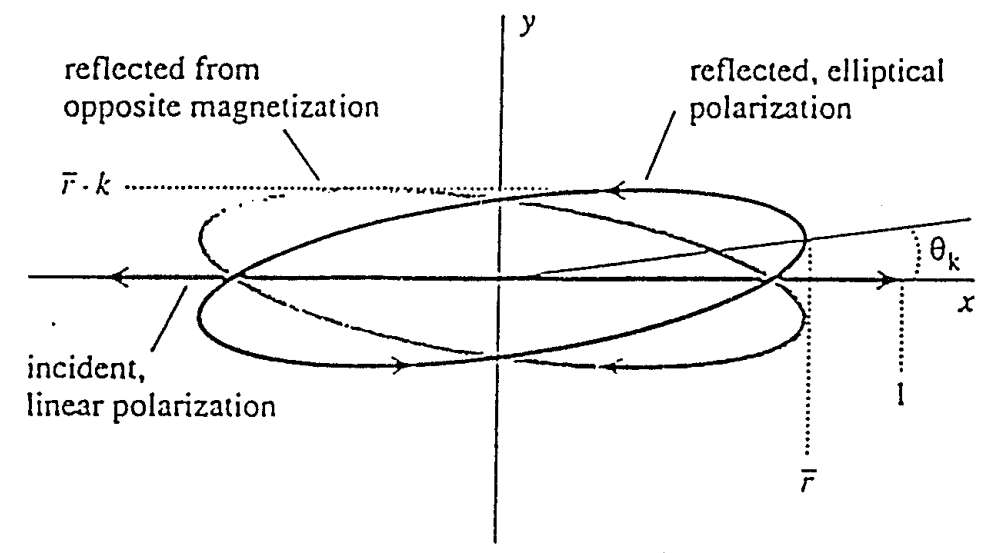

(b)

Figure 3. Principle of MO recording(3) (a) Rotation of a plane polarized beam upon reflection from an MO media, (b) Kerr rotation angle $\left(\theta_{k}\right)$. 
be represented as

$$
I=|r| e^{1 \phi}
$$

where $r$ is the magnitude and $\phi$ is the phase of $r$. Using the indices + and - to denote right and left circularly polarized light respectively, the Kerr rotation angle is given by

$$
\theta_{k}=-\frac{\phi_{+}-\phi_{-}}{2}
$$

and the ellipticity, $\epsilon_{k}$ is

$$
\epsilon_{k}=\frac{\left|r_{+}\right|-\left|r_{-}\right|}{\left|r_{+}\right|+\left|r_{-}\right|}
$$

Magneto-optical recording is discussed in further detail in the following sections.

\subsection{Development of Magneto-optical Recording Media}

The early magneto-optical recording materials were based on MnBi alloys. In the late seventies, materials based on rare earth-transition metal alloys were proposed. More recent investigations include multilayer films, 
polycrystalline garnets, and Heusler alloys. These developments in the magneto-optical recording media are briefly reviewed below.

\subsubsection{History of Magneto-optical Recording}

The discovery of the Faraday effect took place in 1845 when Michael Faraday found that an applied magnetic field affects the propagation of light through glass. In 1888, Kerr discovered a similar magnetic field effect on the rotation of plane-polarized light in the reflection mode.

The use of magneto-optical films for magnetic memory was suggested in 1957.(10) Mayer(11) observed the magnetization reversal in a thin perpendicularly magnetized film of $\mathrm{MnBi}$, upon heating it with the point of a needle. This thermomagnetic effect suggested for magnetic recording used an electron beam for recording. In 1959, Burns ${ }^{(12)}$ proposed the use of the light output of a CRT as a true means of magneto-optical recording. This idea was not practical as it required preheating of the media to temperatures just below the Curie point.

\subsubsection{Early Magneto-optical Recording Materials}

The initial work on magneto-optical recording was primarily focused on $\mathrm{MnBi}$. It offered an unusually large 
Kerr rotation $\left(\theta_{k}=0.7^{\circ}\right)$. Alloys based on $\mathrm{MnBi}$, such as MnCuBi, could be prepared in crystalline form with a curie temperature of about $200^{\circ} \mathrm{C}$. This temperature was high enough for data stability and low enough for acceptable data sensitivity, for the limited laser power available and the substrate stability at that time. However, the fabrication process was quite critical. Also, MnCuBi films are polycrystalline in nature resulting in significant noise in the signal. A detailed study on $\mathrm{MnBi}$ based thin films was done by Chen in 1968.(13) Other early Mo materials surveyed by Lee ${ }^{(14)}$ were also generally polycrystalline. Again, these materials had a grainy optical appearance. The magnetic domains following crystalline boundaries were irregular in shape. All these polycrystalline materials exhibited high readout noise that resulted in a low signal-to-noise ratio (SNR) .

\subsubsection{Recent Magneto-optical Recording Materials}

Amorphous rare earth-transition metal (RE-TM) thin films such as GdCo and GdFe were studied by Chaudhari et al. (15) as materials for magnetic bubble devices. It was pointed out that these materials could be used as magneto-optical media with compensation point writing. This provided a big boost to research activity in the area of magneto-optical storage technology. Subsequently, other RE-TM films such as TbFe, 
GdTbFe, and TbDyFe were prepared. In these cases, curie point recording was employed.(16) It became clear that the amorphous RE-TM alloy films were promising materials for magneto-optical memory. Kryder ${ }^{(17)}$ and Togami(16) have discussed some of the desired materials for magneto-optical recording. Meiklejohn(18) has also evaluated various binary, ternary, and quaternary rare earth-transition metal alloys. of these materials, GdTbCo and TbFeCo were considered to have the best properties for magneto-optical recording. The magnetic and magneto-optical properties of some of the rare earth-transition metal alloys are summarized in Tables 1 and 2 .

Some of the materials recently studied for magneto-optical recording include multilayered film structures such as co/pt and Co/Pd, (19,20) polycrystalline garnets, (21) and Heusler alloys. (22,23) Multilayer films with alternating layers of co and $\mathrm{Pt}$ (or $\mathrm{Pd}$ ) are attractive due to their high perpendicular anisotropy, high chemical stability, and an increase in Kerr rotation at shorter wavelengths. Polycrystalline Bisubstituted garnets also show a larger Faraday rotation at shorter wavelengths, which will be required for future high density recording. Attempts are being made to reduce the large readback noise on these materials. (4) Due to their low perpendicular anisotropy, Heusler alloys such as PtMnSb are still not used as magneto-optical recording media even though these films exhibit a large Kerr rotation (of the order of 


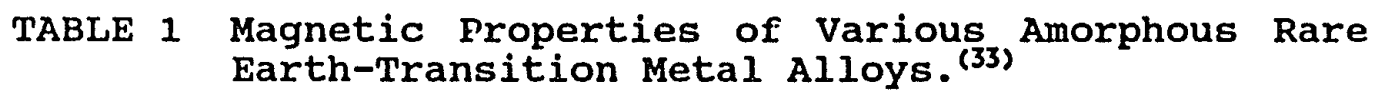

\begin{tabular}{|c|c|c|c|c|c|}
\hline Material & $\begin{array}{l}\text { Curie } \\
\text { temp. } \\
\mathrm{T}_{\mathrm{c}}, \mathrm{o}_{\mathrm{K}}\end{array}$ & $\begin{array}{l}\text { Compen- } \\
\text { sation } \\
\text { temp. } \\
\mathrm{T}_{\text {comp' }}{ }^{\circ} \mathrm{K}\end{array}$ & $\begin{array}{l}\text { Coerc- } \\
\text { ive } \\
\text { energy } \\
\text { densicy } \\
M_{\mathrm{s}^{H}} \mathrm{H}^{\prime} \\
10^{4} \mathrm{j}^{3}\end{array}$ & $\begin{array}{l}\text { Anisotropy } \\
\text { constant, } \\
\mathrm{K}_{\mathrm{u}} \\
10^{4} \mathrm{j} / \mathrm{m}^{3}\end{array}$ & $\begin{array}{l}\text { Kerr } \\
\text { rota- } \\
\text { tion } \\
\theta_{k^{\prime}} \\
\text { dég. }\end{array}$ \\
\hline $\mathrm{Tb}_{23} \mathrm{Fe}_{77}$ & 400 & 300 & 4 & 30 & 0.23 \\
\hline $\mathrm{Tb}_{21} \mathrm{Co}_{79}$ & $\cdots$ & 300 & 4 & 14 & 0.33 \\
\hline $\mathrm{Gd}_{26} \mathrm{Fe}_{74}$ & 480 & 300 & 0.02 & 2.5 & 0.29 \\
\hline $\mathrm{Gd}_{21} \mathrm{CO}_{79}$ & $\cdots$ & 300 & 0.02 & 1 & 0.33 \\
\hline $\mathrm{Tb}_{22} \mathrm{Fe}_{66} \mathrm{Co}_{12}$ & 500 & $<300$ & 8 & 10 & 0.38 \\
\hline $\mathrm{Gd}_{22} \mathrm{~Tb}_{4} \mathrm{Fe}_{74}$ & 450 & 300 & 1.5 & 4 & 0.30 \\
\hline $\mathrm{Gd}_{16} \mathrm{~Tb}_{6} \mathrm{CO}_{78}$ & $\cdots$ & 280 & 1.1 & 4 & 0.32 \\
\hline
\end{tabular}


TABLE 2 Magnetic and Magneto-optical Properties of Various RE-TM Alloys.(16)

\begin{tabular}{|c|c|c|c|c|c|}
\hline Materials & $\begin{array}{l}\text { Record- } \\
\text { ing } \\
\text { Tech. }\end{array}$ & $\begin{array}{l}\text { Curie } \\
\text { temp. } \\
\left({ }^{\circ} \mathrm{C}\right)\end{array}$ & $\begin{array}{l}\text { Figure of } \\
2 \mathrm{~F} / \alpha \\
\left({ }^{\circ}\right)\end{array}$ & $\begin{array}{c}\text { merit } \\
\lambda \\
(\mathrm{nm})\end{array}$ & $\begin{array}{l}\text { Kerr rota- } \\
\text { tion angle } \\
\theta_{k}\left({ }^{\circ}\right)\end{array}$ \\
\hline $\mathrm{MnBi}^{\mathrm{a}}$ & $\mathrm{T}_{c}^{*}$ & 360 & 3.6 & 633 & 0.7 \\
\hline MnCuBi ${ }^{a}$ & $\mathrm{~T}_{\mathrm{c}}$ & 180 & $\begin{array}{l}1.8 \\
1.3\end{array}$ & $\begin{array}{l}515 \\
633\end{array}$ & 0.2 \\
\hline $\operatorname{MnAlGe^{a}}$ & $\mathrm{T}_{\mathrm{c}}$ & 245 & 0.2 & 550 & --- \\
\hline $\mathrm{MnGaGe}^{\mathrm{a}}$ & $\mathrm{T}_{\mathrm{c}}$ & 185 & 0.1 & 850 & --- \\
\hline PtCo ${ }^{a}$ & $\mathrm{~T}_{\mathrm{c}}$ & 390 & $\begin{array}{l}3.3 \\
-5.0\end{array}$ & 633 & -- \\
\hline $\operatorname{GdIG}^{b}$ & $\mathrm{~T}_{\text {comp }}{ }^{\star \star}$ & --- & 2.3 & 633 & -- \\
\hline $\mathrm{GdCo}^{c}$ & $T_{\text {comp }}$ & $>400$ & 0.45 & $\begin{array}{c}450 \\
- \\
820\end{array}$ & 0.33 \\
\hline $\mathrm{GdFe}^{\mathrm{c}}$ & $\mathrm{T}_{\text {comp }}$ & --- & 0.40 & 633 & $0.25-0.58$ \\
\hline $\mathrm{TbFe}^{\mathrm{c}}$ & $\mathrm{T}_{\mathrm{c}}$ & 135 & -- & & $0.25-0.58$ \\
\hline GdTbFe $^{c}$ & $\mathrm{~T}_{\mathrm{c}}$ & $150-165$ & --- & & $0.27-0.52$ \\
\hline TbDyFec & $\mathrm{T}_{c}$ & 75 & -- & & 0.20 \\
\hline
\end{tabular}

\footnotetext{
* $\mathbf{T}_{\mathrm{c}} \quad$ : Curie point recording.

** $\mathrm{T}_{\text {comp }}$ : Compensation point recording.

a : Poly-crystal.

c : Single crystal.

: Amorphous.
} 
two degrees).

\subsubsection{Future Expectations}

It appears that significant progress can be made to achieve higher signal, lower noise, and better environmental stability in magneto-optical thin films. Since the Kerr rotation for amorphous materials is much smaller than, say, crystalline materials such as $\mathrm{MnBi}$ and $\mathrm{Bi}$-substituted garnets, one would think that materials with a high Kerr rotation angle and low media noise coupled with a high perpendicular anisotropy will be developed in future.

\subsection{Desired Characteristics of MO Media}

The magneto-optical media should have a high value of Kerr rotation and an amorphous film structure to enhance signalto-noise ratio. It must have high values of coercivity and perpendicular anisotropy. In addition, the coercivity should decrease with temperature to allow the film to readily switch at the recording temperature. The material parameters that need to be considered for a good Mo media are listed in Table 3. The requirements of a good recording media are discussed below. 
TABLE 3 Properties of Interest for MO Recording Media.(37)

\begin{tabular}{|c|c|c|}
\hline Writing & storage & Reading \\
\hline $\begin{array}{c}\mathrm{T}_{c^{\prime}} \mathbf{T}_{\text {comp' }} \mathbf{M}_{\mathbf{s}} \\
\alpha(\lambda), \mathrm{R}(\lambda) \\
\left(\frac{d H_{c}}{d T}\right)_{T_{\text {cosp }}}\end{array}$ & $\begin{array}{l}H_{c}, M_{s}\left(20^{\circ} \mathrm{C}\right) \\
\Delta H_{c}, \Delta M_{s}(x, T) \\
K_{u}>2 \pi M_{s}^{2}\end{array}$ & $\begin{array}{l}\mu^{\star}=\text {-ik (Temp.) } \\
{[\alpha(\lambda), R(\lambda)]} \\
\text { Thickness }\end{array}$ \\
\hline Thickness & $\begin{array}{l}\text { Magnetostatic } \\
\text { versus wall } \\
\text { energy }\end{array}$ & $\theta_{f}, \theta_{k}, \theta_{f} / \alpha, \theta_{k} R$ \\
\hline Specific heat & Corrosion & $\mathrm{T}_{\max }$ \\
\hline $\begin{array}{l}\text { Thermal } \\
\text { conductivity } \\
\text { (film and } \\
\text { substrate) }\end{array}$ & & Grain structure \\
\hline Spot diameter & & $\begin{array}{l}\text { Interference } \\
\text { layers }\end{array}$ \\
\hline
\end{tabular}




\subsubsection{High Signal-to-Noise Ratio}

The sources of noise in a magneto-optical recording process consist of shot noise, laser noise, electronic noise, and media noise. $(1,17,18)$ From a media standpoint, the goal is to reduce the media noise to a level below the shot noise, assuming the laser and the electronic noises are also below the shot noise level. One possible source of the media noise is the irregularity of the domain. A ragged periphery of the domain will introduce some noise into the system and result in a higher bit shift. This is the reason why amorphous films with mirror-like surfaces produce a better signal-tonoise ratio than polycrystalline MnBi films even though they have a much lower Kerr rotation.

In an ideal Mo system, the limiting source of noise is shot noise in the differential photodiode detectors. In such cases the signal-to-noise ratio is given by

$$
S N R=10 \log \left(2 S P_{o} R \sin ^{2} \theta_{k} / e B\right)
$$

\footnotetext{
where $s$ is the sensitivity of the photodiode,

$P_{0}$ is the incident laser power,

$\mathrm{R}$ is the reflectivity of the disk,

$\theta_{k}$ is the Kerr rotation angle,

e is the electronic charge, and
} 
B is the band width of the detector.

This relationship suggests that the media should be capable of accommodating the high incident beam power for readout without disturbing the written domain, and should have high values of Kerr rotation and reflectivity. Multilayer optical structures to increase $\boldsymbol{\theta}_{k^{\prime}}$ and a nonreflecting coating on the opaque Mo film to increase absorption are suggested to increase the signal-to-noise ratio.(24) However, these techniques have their own limitations.

\subsubsection{High Recording Density or High Bit Density}

Several factors such as the coding scheme, wavelength of the laser, numerical aperture of the lens, and the properties of the recording medium affect the bit density. From the magnetic media standpoint, the smallest domain diameter (see Figure $4^{(25)}$ ) that can be supported in the absence of an applied magnetic field is given by $(17,18)$

$$
\mathrm{d}_{0}=\sigma / \mathrm{M}_{\mathrm{s}} \mathrm{H}_{\mathrm{c}}
$$

where $\sigma=4 \pi\left(A K_{u}\right)^{1 / 2}$, is the domain wall energy,

$M_{s}$ is the saturation magnetization,

$\mathrm{H}_{c}$ is the coercive force of the material,

and $A$ is the exchange constant involved in the 





exchange interaction energy between the magnetic spins,

$\mathrm{K}_{\mathrm{u}}$ is the uniaxial perpendicular anisotropy energy constant related to the orientation of the magnetization and the structure of the film.

Thus, in order to support small domain size, the films need to have a large energy product, $M_{s} H_{c}$, and a minimum domain wall energy, $\sigma$. Minimizing the domain wall energy requires small values of $\mathrm{K}_{\mathrm{u}}$. Therefore, smaller values of $\mathrm{K}_{\mathrm{u}}$ will support smaller domain size, provided the condition, $k_{u}$ $>2 \pi \mathrm{M}_{\mathrm{s}}{ }^{2}$, is maintained. The RE-TM alloys of Tb-Fe exhibit a strong uniaxial perpendicular anisotropy, giving rise to high coercivities. Thus, the stability of small domain size in these materials is not an issue at this time. Domains as small as $70 \mathrm{~nm}$ in diameter have been demonstrated for $\mathrm{TbFe}$ films. (26)

\subsubsection{Low Error Rate and High Data Transfer Rate}

The raw bit error rate of almost all RE-TM alloys is determined by the imperfections in the substrate and the media rather than by fundamental magneto-optical SNR (signalto-noise ratio). An error rate of $10^{-12}$ requires an SNR of only $20 \mathrm{~dB},{ }^{(17)}$ which is far less than the SNR value of over $35 \mathrm{~dB}$ that has already been achieved.

The data transfer rate for magneto-optical systems is 
limited by the thermomagnetic recording process rather than the characteristics of the recording media. The limited power of the semiconductor laser, and the requirement of the media to pass the optical head twice for overwrite (once to erase data and once to write data) are the controlling factors for a high data transfer rate. (26)

\subsection{4 overwrite Capability}

Currently, two passes over the media are needed to overwrite the old data with new data. The tracks needed to be written over are heated by a laser beam. During the first pass, a bias magnetic field is applied pointing in the erasing direction. During the following pass the bias magnetic field is switched back to the writing direction, and new data is written. During magneto-optical recording process, a large electromagnet is required due to a large head-to-disk spacing. The electromagnet is too large to be switched at the high frequencies required for high data transfer rates. This is one of the major disadvantages of optical recording as compared to magnetic recording. Due to the importance of direct overwrite, a great deal of work has been done in devising hardware and media-based direct overwrite schemes. (26) 


\subsubsection{Media Cyclability}

Cyclability is one of the major problems with phase-change media, preventing this class of materials from becoming the media of choice for reversible optical storage technology. (3) However, it is not considered to be a major problem in magneto-optical recording media. Thin magneto-optical films are reported to withstand over $10^{10}$ write-erase cycles with less than $10 \%$ change in minimum stable domain size. (17,27) For most of the applications, $10^{6}$ cycles are considered to be sufficient. (3)

\subsubsection{Data Stability}

Data stability means that the information recorded on the media does not change with time. This may involve various considerations. For example, the coercivity of the media at room temperature should be large enough to avoid the accidental erasure of the recorded information. (2) There should not be any change in the media large enough to destroy the recorded data. This means that the media is chemically stable so that it does not corrode or oxidize, and there are no physical changes in the media that would affect the recorded information.

Amorphous phases are metastable and would tend to crystallize at higher temperatures. RE-TM films are 
amorphous in nature. The temperature for recrystallization of the RE-TM films should be high. This will enable these films not to recrystallize at the writing temperature or at room temperature. $\operatorname{Kryder}^{(17)}$ estimated the life time for amorphous-to-crystalline phase transition for GdCo films as given in Table 4 . These results demonstrate that these materials would not readily crystallize at room temperature, and are suitable for magneto-optical recording.

\subsection{Properties of Thin RE-TM alloy films}

As discussed earlier, the amorphous thin films of rare earth-transition metal alloys are the most promising of various magneto-optical media. This work aims at studying the oxidation-induced changes in the RE-TM alloys. In order to understand the magneto-optical recording process, the magnetic and magneto-optical properties of these alloys will be briefly reviewed. This knowledge will also be helpful in understanding the effect of oxidation on the properties of these RE-TM alloy films.

\subsubsection{Film Microstructure}

As mentioned earlier in section 2.3.3, the RE-TM films are amorphous over a wide range of compositions. These amorphous films were obtained by different sputtering methods, (28) e.g., 
TABLE 4 Lifetime of Amorphous Films before Crystallization. (17)

\begin{tabular}{lll}
\hline $\begin{array}{l}\text { Temperature } \\
\left({ }^{\circ} \mathrm{C}\right)\end{array}$ & $\begin{array}{l}\text { Amorphous-to-crystalline } \\
\text { transformation (years) }\end{array}$ & $\begin{array}{l}108 \text { change in } \\
\mathrm{K}_{\mathrm{u}}\end{array}$ \\
\hline & & \\
100 & 0.06 & 60 days \\
50 & 100 & 70 years \\
25 & & 3000 years \\
\hline
\end{tabular}


diode sputtering and DC magnetron sputtering. Also, these films remained amorphous even with changes in some of the processing variables during deposition. For example, different gas pressures, bias voltages, (25) and film deposition angles(29) were used to make amorphous films of rare earth-transition metal alloys. Films deposited by different methods, however, exhibited quite different microstructure, morphology, and density.

The amorphous nature of these films offered various advantages. These films had low media noise that resulted in a high signal-to-noise ratio. The films were less susceptible to corrosion due to the absence of a grain boundary area. The low resistivity of these films limited the eddy currents during writing. The films also had good thermal stability.(3)

\subsubsection{Magnetization versus Temperature and Concentration of RE Element in the Film}

The magnetic properties of RE-TM alloys are ferrimagnetic in nature. The rare earth and the transition metal subnetworks are coupled antiferromagnetically. This means that the contributions of the magnetization from the rare earth and the transition metal components oppose each other, as shown in Figure 5. At lower temperatures, the magnetization component from the rare earth element, $M_{R E}$ is 


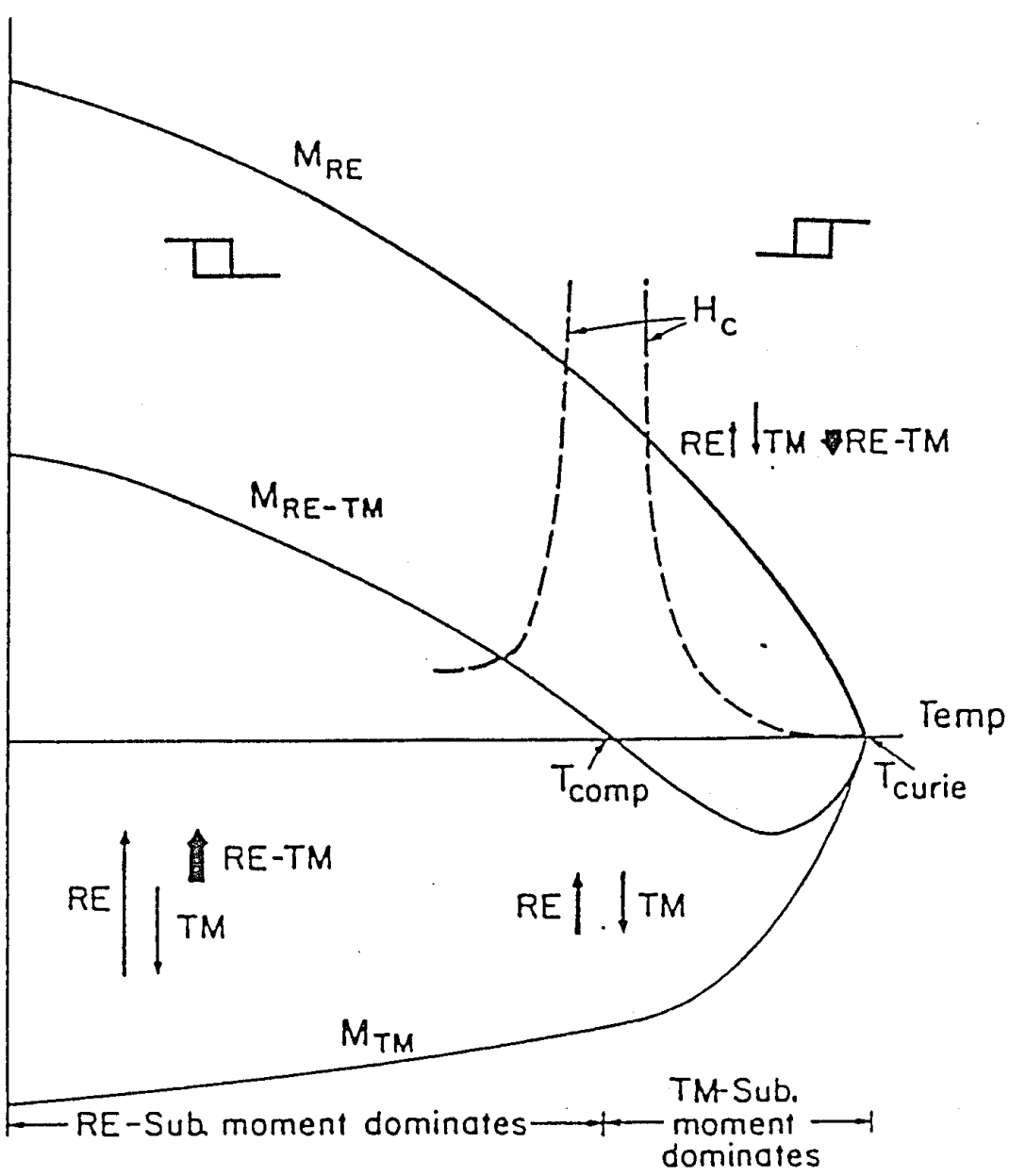

Ferrimagnetic, anti ferromagnetically coupled

Figure 5. Temperature dependence of magnetization and coercivity for an RE-TM alloy.(25) 
higher than that from the transition metal, $M_{T H}$. As the temperature of the film is increased, the magnetization component from each of the subnetworks decreases. However, the rate of decrease in the magnetization is less for the transition metal than for the rare earth element. Since the two components act in the opposite directions, the net value of the magnetization in the film will be zero at a certain temperature. This temperature is called the compensation point, $T_{\text {comp }}$ for the RE-TM film. It is obvious that, depending upon the composition of the film, the compensation point may be below or above the room temperature. However, for a film composition with the percentage of the rare earth element greater than a certain value, the magnetization component of the transition metal may not become equal to that of the rare earth element at any temperature. This means that these alloys will not exhibit a compensation point. The dependence of the compensation point on the composition of the film is further illustrated in Figure 6 . It shows a change in the net magnetization with temperature for 19, 23, and 28 atomic percent $T b$ in TbFe films. This figure shows that the compensation point may be far below the room temperature for low Tb concentrations (19 at. $\%$ curve), close to the room temperature for intermediate $\mathrm{Tb}$ concentrations, (23 at. $\%$ curve), and may not be evident for high $\mathrm{Tb}$ concentrations (28 at. 8 curve). Also, note that the Kerr loop orientation is reversed as the $\mathrm{Tb}$ concentration is 


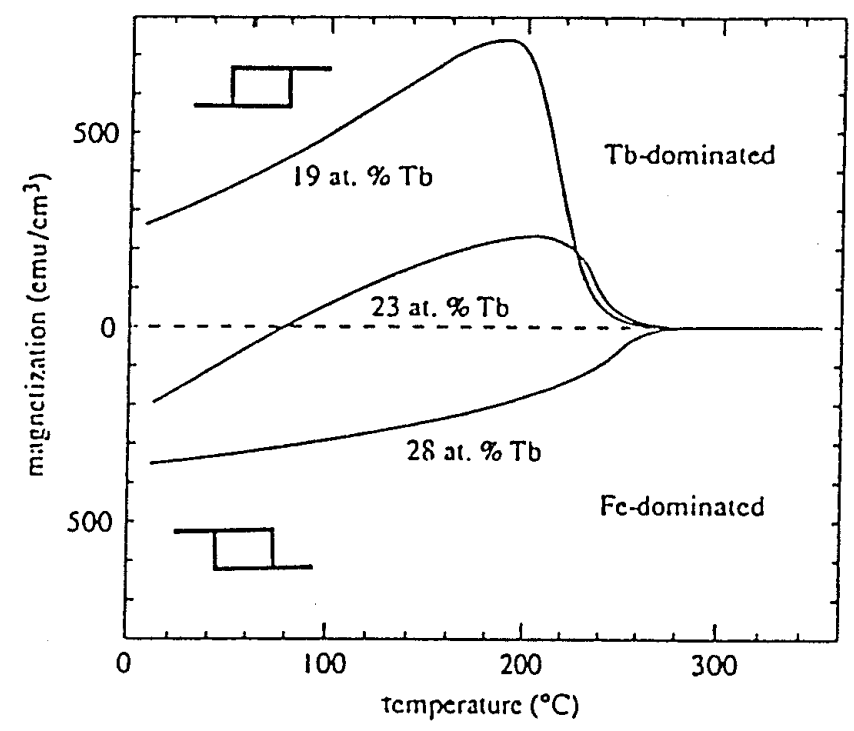

Figure 6. Effect of $\mathrm{Tb}$ concentration on compensation temperature through change in net magnetization. (3) 
increased from 19 to 28 atomic percent.

If the temperature of the alloy film is further increased above the compensation temperature, the magnetization component due to the transition metal, $\mathbf{M}_{T H}$, will become higher than the component due to the rare earth element, $M_{R E}$. This will result in a net magnetization. The direction of the net magnetization above $T_{\text {comp }}$ will be opposite to the net magnetization direction below $T_{\text {comp }}$. With an increase in temperature, the magnitude of the net magnetization increases at first. However, as the film temperature approaches a certain value called the curie point, $\mathrm{T}_{\text {curie' }}$ the net magnetization in the film rapidly decreases to zero. It must be remembered, however, that the rare earth and transition metal components exhibit a co-operative magnetic behavior. This means that the contribution of each component to the net magnetization in the film is affected by the presence of the other component. Thus, as the curie temperature is approached, the magnetization of the individual components due to the transition metal and the rare earth element, and the net magnetization in the film decrease to zero. These changes are shown in Figure 5 .

2.5.3 Effect of Temperature and RE Concentration on Coercivity

The effect of the Tb concentration in RE-TM films on the 
compensation point was briefly discussed above. The effect of the concentration of $\mathrm{Tb}$ on the temperature dependence of coercivity for TbFe films was studied(31) and is shown in Figure 7. The increase in $\mathrm{Tb}$ concentration resulted in raising the compensation point to higher temperatures. As shown in the figure, the compensation point may not be apparent for RE-TM alloy films with high Tb concentration.

The change in the coercivity of these films in the vicinity of the compensation temperature, $T_{\text {comp' }}$ is also shown in the same figure. As the compensation temperature was approached from either side of the $\mathrm{T}_{\text {comp' }}$ coercivity of these films increased towards infinity. This large increase in the coercive force is not due to the magnetization having to overcome a potential barrier, but rather because the net magnetization, $M$, tends towards zero. The torque on the net magnetization, magnetostatic energy, $-\mathrm{MH}$, which acts to move the domain wall, also tends toward zero. As the films were raised to the curie temperature, the coercivity decreased to zero. At temperatures lower than $\mathrm{T}_{\text {comp' }}$ coercivity decreased to a minimum before it started to increase again. The minimum value of the coercivity increased with a decrease in the compensation temperature. When no compensation point existed, as for the films with high $\mathrm{Tb}$ concentration, the coercivity exhibited an unusual temperature dependent behavior that will not be discussed here.

The effect of the rare earth content on the compensation 


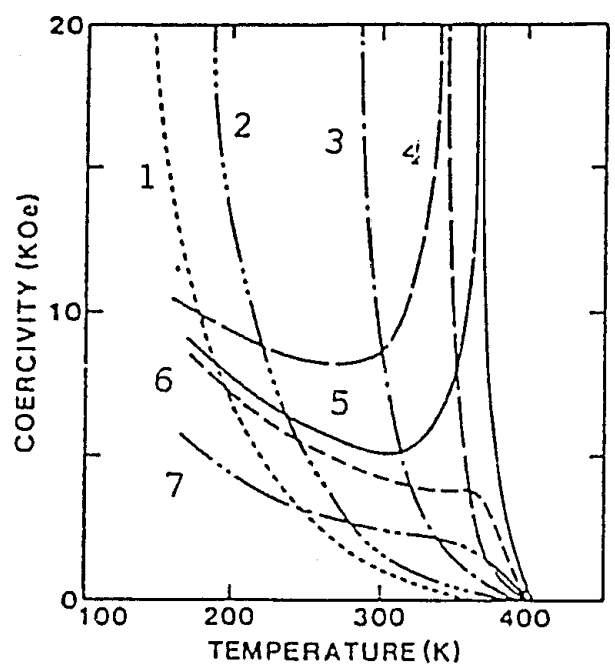

$\begin{array}{ll}1 & \mathrm{~Tb}_{18} \mathrm{Fe}_{72} \\ 2 & \mathrm{~Tb}_{20} \mathrm{Fe}_{80} \\ 3 & \mathrm{~Tb}_{23} \mathrm{Fe}_{77} \\ 4 & \mathrm{~Tb}_{25} \mathrm{Fe}_{75} \\ 5 & \mathrm{~Tb}_{27} \mathrm{Fe}_{73} \\ 6 & \mathrm{~Tb}_{29} \mathrm{Fe} e_{71} \\ 7 & \mathrm{~Tb}_{32} \mathrm{Fe} e_{68}\end{array}$

Figure 7. Coercivity versus temperature for $\mathrm{Tb}_{x} \mathrm{Fe}_{y}$
films.(3i) 
temperature and the curie temperature of different RE-TM alloys films was also studied. (32) The results obtained are shown in Figure 8. The concentration of the rare earth element in the films had a very strong effect on the compensation temperature, $T_{\text {comp }}$. The compensation temperature increased with an increase in the RE element concentration. However, the curie temperature was found to be far less sensitive to the concentration of the rare earth element in the film. The curie temperature of the RE-TM films can be adjusted by substituting for the base transition metal and/or rare earth elements in ternary and quaternary alloys. Tsujimoto et al. (34) have studied the effect of substituting Co for $\mathrm{Fe}$ in $\mathrm{Tb}_{21}\left(\mathrm{Fe}_{100-\mathrm{x}} \mathrm{Co}_{\mathrm{x}}\right)_{79}$ alloys on coercivity and magnetization. It was seen that the curie temperature of these films increased from about $90^{\circ} \mathrm{C}$ to $150^{\circ} \mathrm{C}$ when $\mathrm{x}$ was increased from 0 to 15 .

\subsubsection{Effect of Film Composition and Temperature on Kerr Rotation}

As already mentioned, the curie temperature may change with the alloy composition. The Kerr rotation of various rare earth substituted FeCo films was plotted against the Curie temperature, as shown in Figure $8 \mathrm{~d}^{\text {(33) }}$ It showed that the increase in Kerr rotation angle at room temperature could be related to the increase in Curie temperature of the film. 


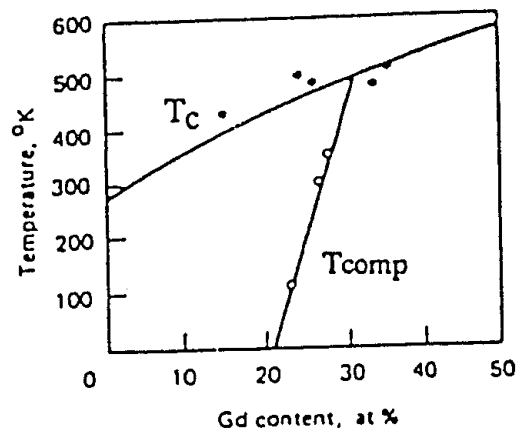

(a)

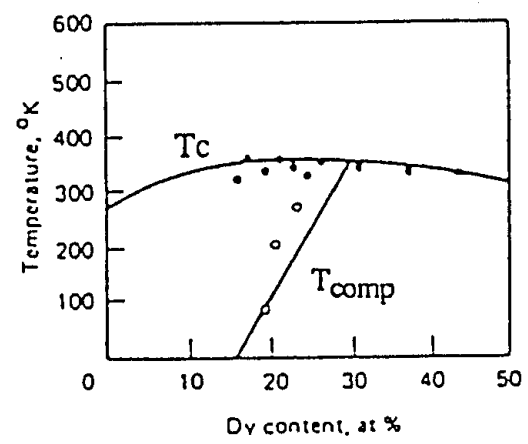

(c)

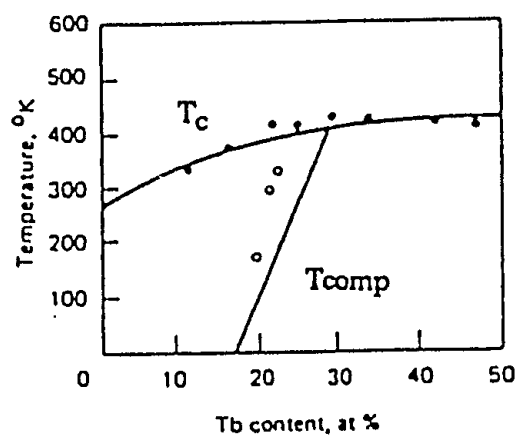

(b)

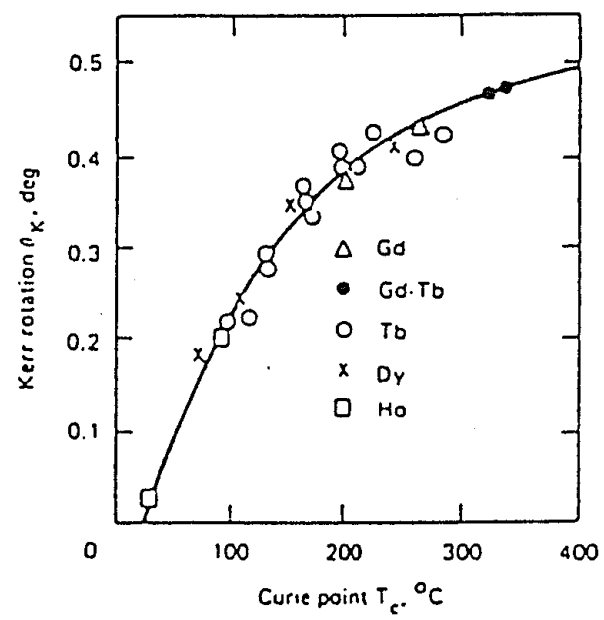

(d)

Figure 8. Effect of RE concentration on compensation and Curie temperature for RE-Fe films. (a) Gd, (b) $\mathrm{Tb}$, (c) Dy. Effect of Curie temperature on Kerr rotation of various REiM alloys (d). (33) 
The study done by Tsuzimoto et al. ${ }^{(34)}$ indicated that with an increase in the temperature of RE-TM films, the Kerr rotation angle decreased slowly at first. It decreased to zero rapidly as the temperature was increased to the curie point. The study also showed a higher value of Kerr rotation angle for $\mathrm{Tb}_{21}\left(\mathrm{Fe}_{100-\mathrm{x}} \mathrm{Co}_{\mathrm{x}}\right)_{79}$ alloys with increasing value of $\mathrm{x}$, i.e., increasing the co concentration in the film increased the Kerr rotation for the film at room temperature as well as at $70^{\circ} \mathrm{C}$. An increase in $x$ from 0 to 30 resulted in an increase from $0.1^{\circ}$ to $0.3^{\circ}$ in the Kerr rotation angle measured at room temperature. Other authors ${ }^{(17,38)}$ have also pointed out the higher value of Kerr rotation angle in TbFeco films as compared to TbFe films. This enhancement in the Kerr rotation angle with the addition of co will also be discussed later on the basis of the results obtained from this study. Magnetic and magneto-optical properties of various rare earth-transition metal alloys can be seen in Table 1 and Table . $^{(16,33)}$

\subsubsection{Magnetic Anisotropy}

An MO material should have a large value of intrinsic perpendicular uniaxial anisotropy energy density, $\mathrm{K}_{\mathrm{u}}$ (35) Magneto-optical recording requires a media with a positive effective anisotropy. This means that in order to use normal incidence Kerr rotation for reading the signal, it is 
necessary that the easy axis of magnetization for the recording media be normal to the film plane. Also, a strong perpendicular anisotropy gives rise to a high value of coercivity. (36) A high value of coercivity is required for a sub-micron domain to be stable in a zero external magnetic field.

The origin of perpendicular anisotropy in thin RE-TM films has been attributed to several factors including shape (growth morphology), magnetostriction due to strain applied to the MO film by the substrate, exchange anisotropy, and pair ordering, $(18,37)$ etc. Recently, however, Marinero and coworkers $^{(38,39)}$ have concluded that the magnetic anisotropy may arise either from a macroscopic structural anisotropy in these films, or as a consequence of the energetic bombardment of the growing films. The origin of the anisotropy for Mo materials is very important. The most desirable source of $\mathrm{K}_{\mathrm{u}}$ is pair ordering and columnar growth. The least desirable source for this anisotropy is the strain in the magnetic film. In this case $K_{u}$ may change with temperature or the film may lift from the substrate. Both of these cases are highly undesirable.

2.6 Write, Read, and Erasure Processes for Magnetooptical Recording Materials

The properties of the rare earth-transition metal alloys 
have been discussed in previous sections. These properties affect the writing, reading, and erasure processes for magneto-optical recording. The process of writing is achieved by thermomagnetic means, and the Kerr effect is used during the readout process. The schematic for these processes is shown in Figure 9.(4) Also, the material properties of interest for reading, writing, and storage of information are summarized in Table 3.(37) A short description of the writing, reading, and erasure processes in these materials is given below.

\subsubsection{The Writing Process}

For magneto-optical recording, the recording media composition is adjusted such that the films have very high coercivity of several koe at room temperatures and a low coercivity at higher temperatures. Such a media results in a high data stability at room temperatures. For writing, a diffraction limited spot on the film is heated by a focused laser beam of moderately high intensity in the presence of a magnetic field. The magnetic field is applied antiparallel to the direction of magnetization in the film. Typically, the numerical aperture of the lens is such that the spot has sub-micron dimensions. The temperature dependence of the coercivity of the RE-TM metal films has been discussed earlier with reference to Figure 5. With an increase in the 

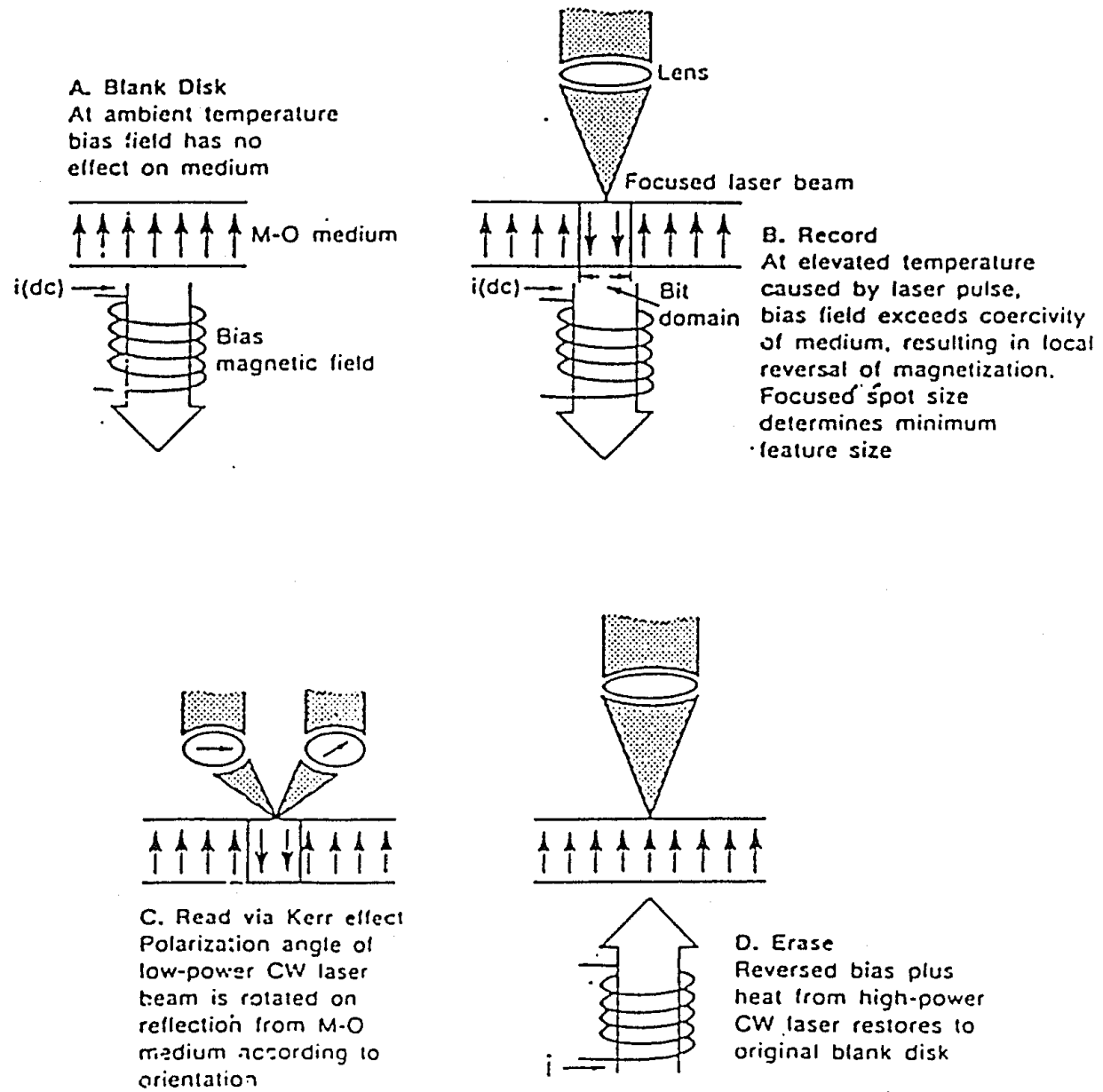

\section{Figure 9. Writing, reading, and erasing processes for MO recording. ${ }^{(4)}$}


film temperature, the coercivity of the localized spot is decreased, so that at the switching temperature the coercivity is much less than the applied field. Therefore, the magnetization in the locally heated spot will switch to the direction of the applied field and will remain in that direction as the spot cools down to room temperature. The applied field is much less than the coercivity of the media at room temperature. Therefore, the magnetization on the unheated area of the disk remains the same. Thus, by pulsing the laser in the presence of a constant magnetic field applied antiparallel to the magnetization direction in the media, data may be written onto the disk. It is also possible to write on a randomly magnetized media by changing the direction of the magnetic field in synchrony with the laser. Writing on the pre-existing information, overwrite, requires two rotations of the disk. The first rotation is needed for erasing the data and the second for writing the bit. Some techniques are currently being developed for one pass overwrite on the magneto-optical recording media.(41)

\subsubsection{The Reading Process}

The readback process utilizes the Magneto-optical Kerr effect. During the readout process the laser power is reduced below the write threshold. Upon reflection from the magnetic film, the plane of polarization of the incident 
light is rotated clockwise or counter-clockwise. This depends on whether the magnetization in the spot being read is parallel or antiparallel to the incident beam. After correcting for any ellipticity introduced by the Mo effect, the light is passed through a polarizing beam splitter set at $45^{\circ}$ to the incident light beam polarization. The intensities of the two split beams is measured using photodiodes. With no magneto-optical rotation of the plane, the intensity of light at each photodiode is the same, resulting in a zero difference signal. With rotation of the plane of polarization, one photodiode receives more light than the other. The difference in the two signals corresponds to the Kerr rotation, and the polarity of the difference signal indicates the direction of magnetization.

\subsubsection{The Erasure Process}

The erasure process may be accomplished in two ways. First, the laser beam may be pulsed with an energy level corresponding to the write process with the applied magnetic field in a direction opposite to the direction of magnetization. This will erase the bit. In the second method, a large magnetic field compared to the room temperature coercivity of the media may be used. 


\subsection{Aging and Chemical stability of RE-TM Films}

The stability of the magneto-optical materials against long term aging and corrosion is one of the main considerations involved in selecting a media for recording applications. Magnetic rare earth elements react strongly with the oxygen in water or air to form non-magnetic oxides. Similarly, iron rusts rather quickly when exposed to the atmosphere. $(17,18,33,37,42)$ This environmental degradation may deteriorate the thermomagnetic and magneto-optical properties of the media to a point that errors will occur in the readback signal. The determination of the chemical stability of an amorphous film over a long period is difficult. Accelerated life tests are performed to estimate the useful lifetime of the recording media. The tests are generally carried out at increased temperatures and an aggressive test environment, such as increased humidity, using corrosive gases, etc. Various properties of the media, such as coercivity, magnetization, Kerr rotation, perpendicular anisotropy, changes in CNR (carrier-to-noise ratio) and BER (bit error rate), background noise level, and chemical composition may be used to determine the rate of degradation of the media due to corrosion. Two approaches have been pursued to improve the corrosion resistance of the media. One approach involves using additives to the Mo media, and the other is based upon using protective layers to passivate 
the RE-TM alloy thin films. The oxidation behavior of these films and various protection methods under investigation are discussed below.

\subsubsection{Oxidation Behavior of RE-TM Media}

In the past, several studies have been conducted in order to determine the environmental stability of the rare earthtransition metal alloys. These studies indicate that the principal mechanism of degradation of RE-TM alloys is oxidation. The rare earth element has a stronger affinity for oxygen than the transition metals, and hence tends to oxidize preferentially. $(43,44,45,46)$

Allen et al. (43) pointed out the formation of a thin initial oxide layer, of about $50 \AA$ thickness, in dry air at room temperature. The Kerr rotation and ellipsometry measurements were used to study the kinetics of this oxidation process in TbFe films at $22^{\circ} \mathrm{C}, 71^{\circ} \mathrm{C}, 100^{\circ} \mathrm{C}$, and $130^{\circ} \mathrm{C}$. Figure 10 shows that the oxide thickness in these films grew with third root of time (minutes ${ }^{1 / 3}$ ), for oxide thicknesses between $50 \AA$ and $300 \AA$. This oxide growth was considered to take place due to field-enhanced diffusion of oxygen or $\mathrm{Tb}$ and $\mathrm{Fe}$. Two distinct surface layers were found to exist. The first layer was the oxide layer where the composition was constant throughout the thickness of the layer, and the second layer was an intermediate layer where 


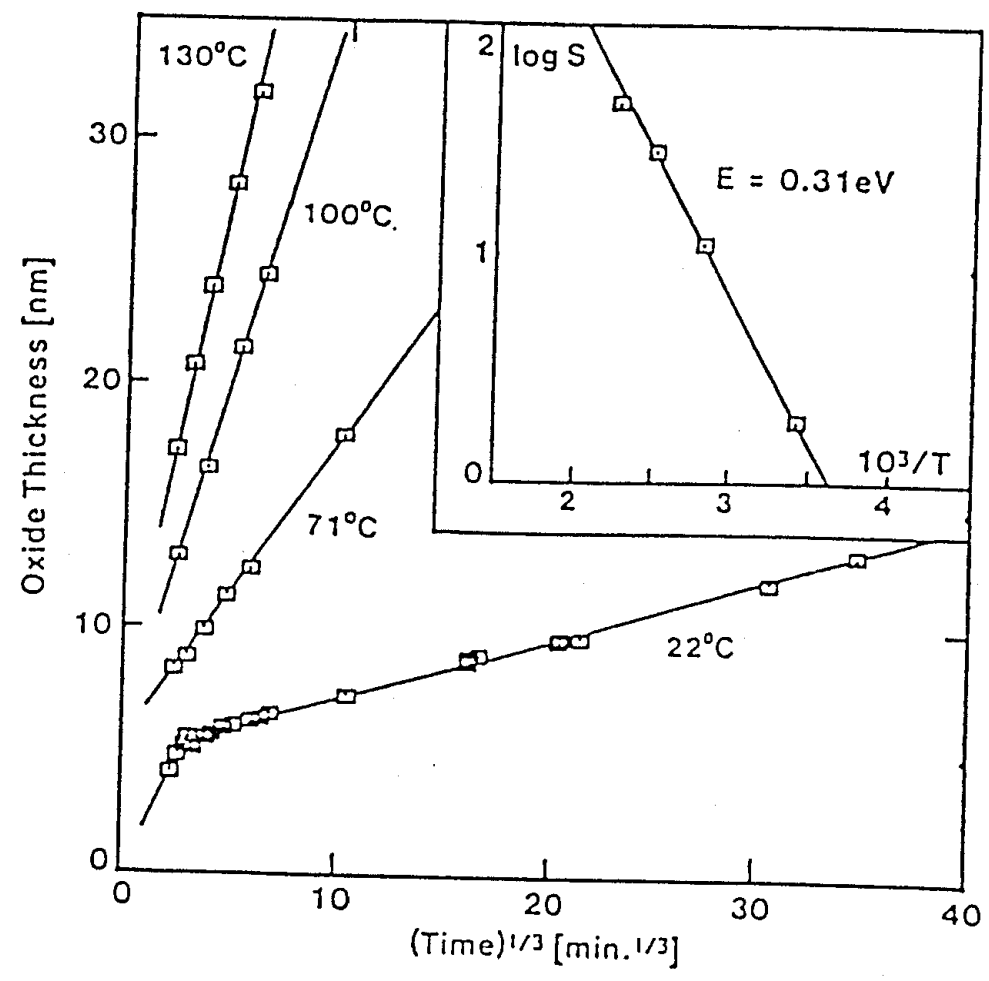

Figure 10. Oxide formation with annealing time for TbFe films. ${ }^{(43)}$ 
the concentration of $\mathrm{Tb}, \mathrm{Fe}$, and oxygen varied with film thickness. This is shown in Figure 11. The oxide layers on the surface were found to be Tb rich and Fe deficient, and the intermediate layer was found to be Fe rich and $\mathrm{Tb}$ deficient relative to the bulk of the film. This oxidation behavior of these films was suggested to be due to the preferential diffusion of $T b$ through the film and its oxidation at the surface.

Using the active TbFe layer sandwiched between varying thicknesses of $\mathrm{Tb}$, Bernstein et al. (44)also attributed the aging phenomenon in the RE-TM films to the migration of $\mathrm{Tb}$ atoms towards the surface. The $\mathrm{Tb}$ was found to react with oxygen from the $\mathrm{SiO}_{2}$ protective overcoat. A recent study on interfacial stability of $\mathrm{TbFeCo}$, with $\mathrm{Al}_{2} \mathrm{O}_{3}$ protective layers and substrates, (42) demonstrated the migration of $\mathrm{Tb}$ to the interfaces. Also, oxygen from the glass substrate and the $\mathrm{Al}_{2} \mathrm{O}_{3}$ protective layer migrated to the MO film. This showed that the glass and protective oxide overcoats may serve as potential sources of oxygen, and the rare earth elements have a strong propensity to form oxides.

oxidation of TbFe thin films has also been studied by Van Dover et al. (45) The oxidation process for these bare films was rather complex. Two surface layers were quickly formed at room temperature. The top surface layer, of $\mathrm{Fe}_{2} \mathrm{O}_{3}$, was about $20 \AA$ thick. The layer underneath was of terbium oxide and was measured to be about $60 \AA$. This terbium oxide layer 

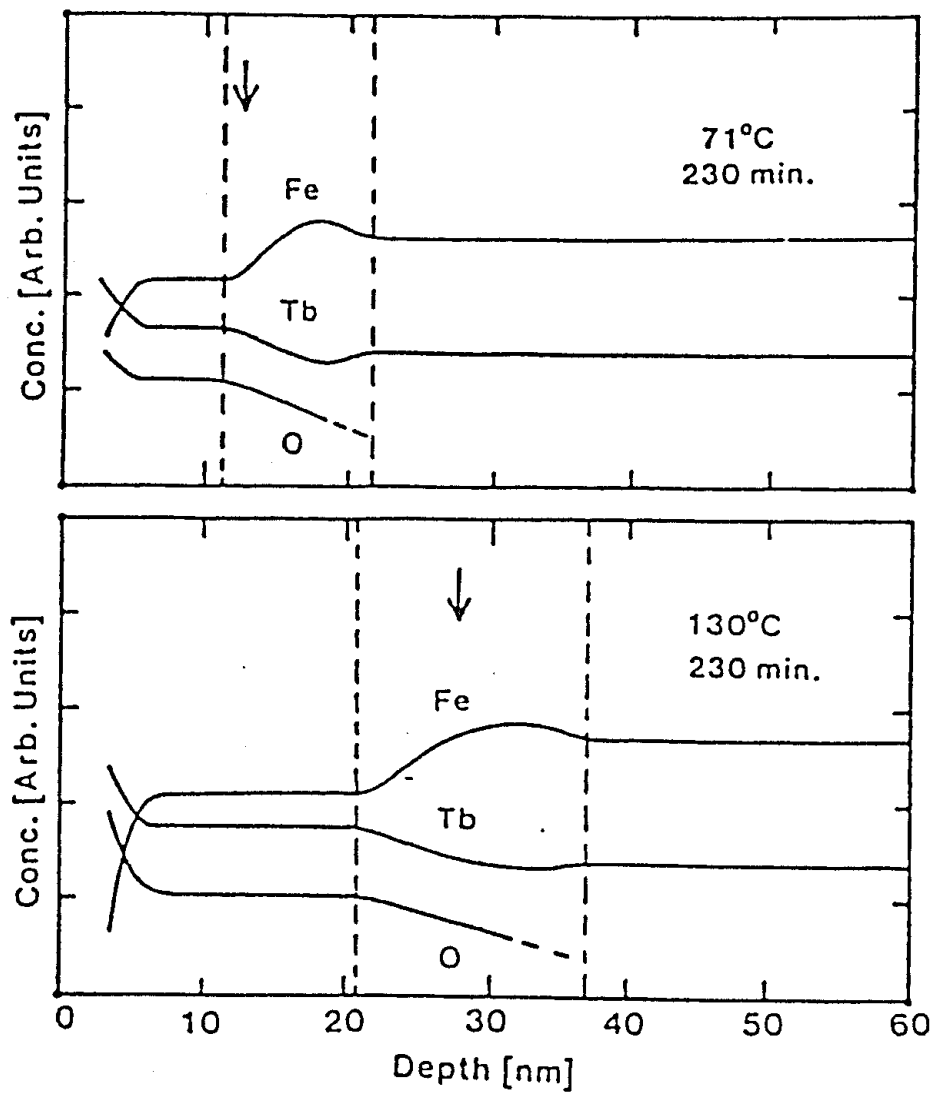

Figure 1.1. The changes in composition of TbFe films with depth, due to oxidation. (43) 
was nonstoichiometric $\mathrm{Tb}_{2} \mathrm{O}_{3}$ or $\mathrm{TbO}_{(1.5+\mathrm{x})}$. Both of these layers, shown in Figure 12 were formed at room temperature and did not grow when the film was heated at $200^{\circ} \mathrm{C}$ for more than 40 hours.

Upon heating at $200^{\circ} \mathrm{C}$, another mixed layer containing $\mathrm{Tb}$, 0 , and $\mathrm{Fe}$, was formed underneath the layers discussed above. In this layer, $\mathrm{Fe}$ was found to be in an unoxidized form and all oxygen was assumed to be bonded to $\mathrm{Tb}$. The $\mathrm{O} / \mathrm{Tb}$ ratio indicated this terbium oxide to be a lower oxide than $\mathrm{Tb}_{2} \mathrm{O}_{3}$. Magnetic measurements indicated that some of the $\mathrm{Tb}$ existed in the metallic form. The ratio of the metallic Tb to Fe was estimated to be about $10 \%$. Below this oxidized zone was the unoxidized zone where the film had the same $\mathrm{Tb} / \mathrm{Fe}$ ratio as in the original film. After the growth of the thin surface oxides, the oxidation zone grew at the expense of the unoxidized zone. The composition of the oxidation zone did not change until it reached the substrate. Then the surface oxides started growing at the expense of the oxidation zone. This study concluded that the oxidation process was governed by the parabolic growth law, as shown in Figure 13, pointing to the possibility of an oxidation process that is limited by the thermal diffusion of oxygen.

\subsubsection{Oxidation Protection for MO Media}

Due to the high tendency of the RE-TM alloy films for 
Oxidation time

at $200^{\circ} \mathrm{C}$

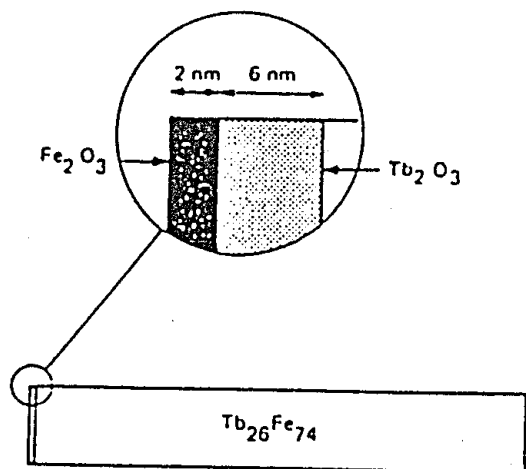

$1 \mathrm{~h}$

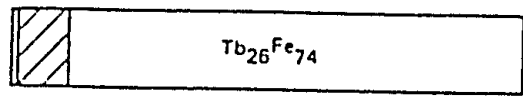

$\mathrm{Tb}_{7} \mathrm{Fe}_{93}+$
$\mathrm{TbO}, 5_{-x}$

$5 \mathrm{~h}$

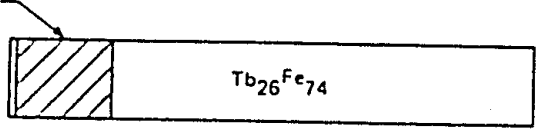

$31 \mathrm{~h}$

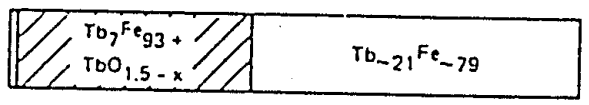

$90 \mathrm{~h}$

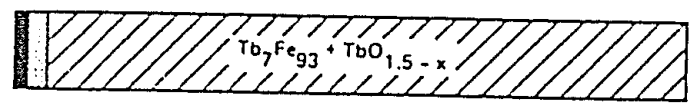

$257 \mathrm{~h}$
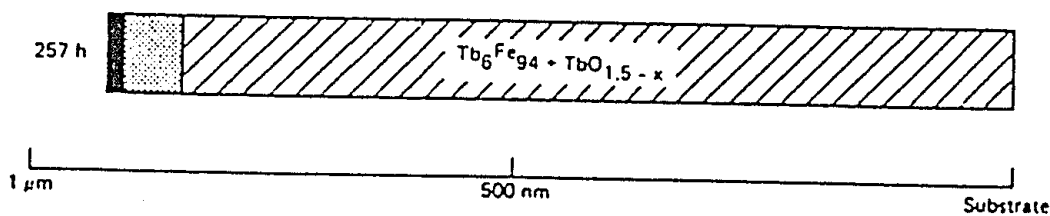

Figure 12. Formation of a complex film structure during oxidation of a TbFe film at $200^{\circ} \mathrm{C}$ for 5 hours. (45) 


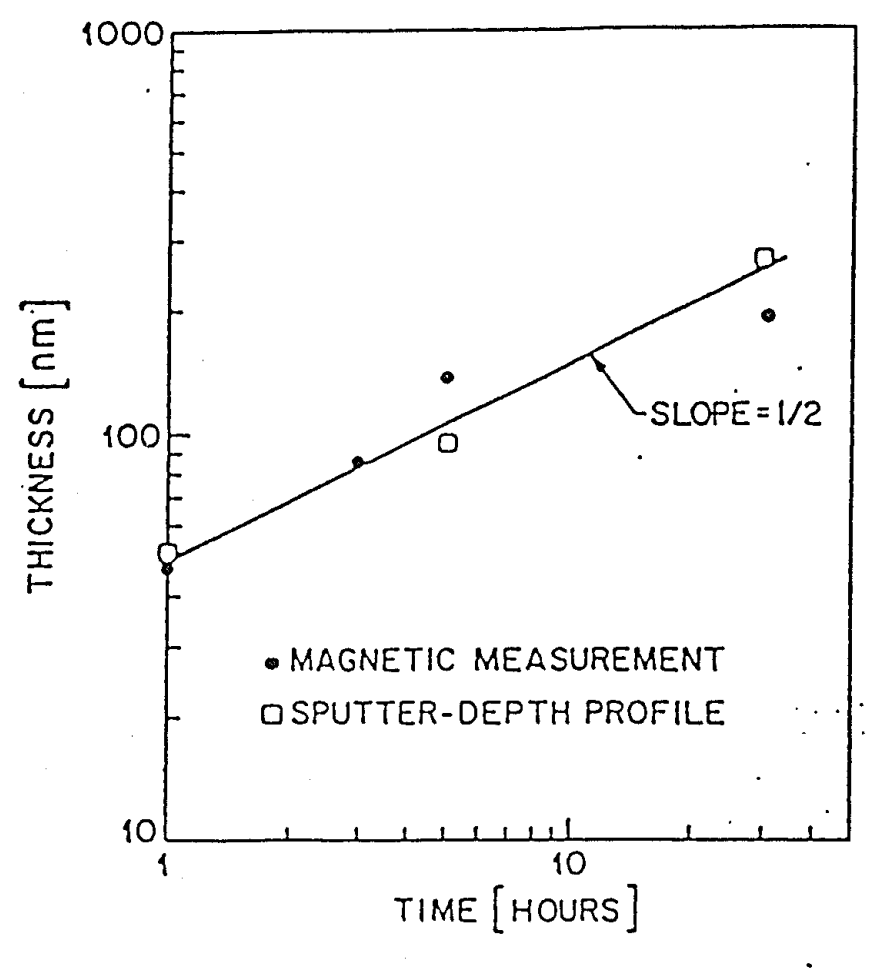

Figure 13. Parabolic growth rate oxidation process for

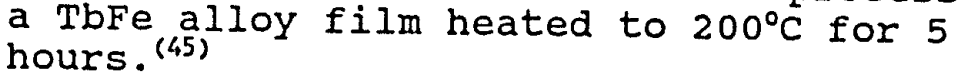


oxidation, various researchers have looked for ways to protect these films from environmental degradation. Changing process parameters during film deposition, use of additive alloying elements, and use of protective dielectric layers are some of the methods suggested for improving the life of the Mo films.

Nakahara et al. (29) and Hong et al.(47) studied the effect of deposition method and process parameters on microstructure and aging behavior of RE-TM films. It was found that in contrast to the diode-sputtered TbFeco films, the perpendicular anisotropy of magnetron sputtered films was much more stable at $300^{\circ} \mathrm{K}$. Also, the films prepared by magnetron sputtering were found to be dense and smooth as compared to the columnar structure exhibited by the diode sputtered films. This was attributed to the lower Ar pressure used in the magnetron sputtering process. In both of the sputtering techniques, the films prepared at lower $\mathrm{Ar}$ pressures exhibited a more dense structure, accompanied by a significant reduction in the void region and an increase in the film grain size. Shieh(25) and Luborsky et al. (48) have also shown that the lower Ar pressure and bias voltage lead to more dense and oxidation-resistant films.

A number of additives have been shown to improve the oxidation resistance of TbFe films. Alloying elements that will form a self passivation layer are expected to result in a more stable film. Addition of co has been reported ${ }^{(3,49)}$ to 
improve the stability of TbFe films because of the formation of a passive layer. Imamura et al.(49) found an improvement in the corrosion resistance and suppression of pinhole formation for TbFe films with the addition of various alloying elements. Figure 14 illustrates the effect of the addition of alloying elements on transmittance of TbFe films subjected to corrosion in aggressive environments. Some of these elements, e.g., Co, Pt, and $\mathrm{Ni}$ also enhance the Kerr rotation. Thus, based on the kerr rotation and media stability point of view, TbFecopt alloys may offer the best overall performance characteristics. A significant improvement in the resistance to oxidation of indium-doped TbFe films due to the interaction of indium with the oxides on the surface has been demonstrated. (52)

Studies have been done on the use of different overcoats for RE-TM films to extend their useful life. The overcoats can significantly reduce oxidation and aging. Due to its strong affinity for oxygen, $T b$ tends to reduce an otherwise stable oxide at the interface. For example, AlN is reported to protect the changes in the magnetic properties of these films much better than does $\mathrm{SiO}_{2}{ }^{(18)}$ The mixed oxides based on $\mathrm{Al}_{2} \mathrm{O}_{3}{ }^{(51)}$ (such as $\mathrm{Al}_{2} \mathrm{O}_{3}-\mathrm{Ta}_{2} \mathrm{O}_{5}, \mathrm{Al}_{2} \mathrm{O}_{3}-\mathrm{TiO}_{2}, \mathrm{Al}_{2} \mathrm{O}_{3}-\mathrm{SiO}_{2}$ ), AlsiN, and Alsion ${ }^{(52)}$ films are reported to be some of the most suitable protection layers. Various other overcoats such as Zns, $\mathrm{Al}_{2} \mathrm{O}_{3}$, sio, $\mathrm{TiO}_{2}$ etc., have been found to offer varying degree of protection to the magneto-optical recording 


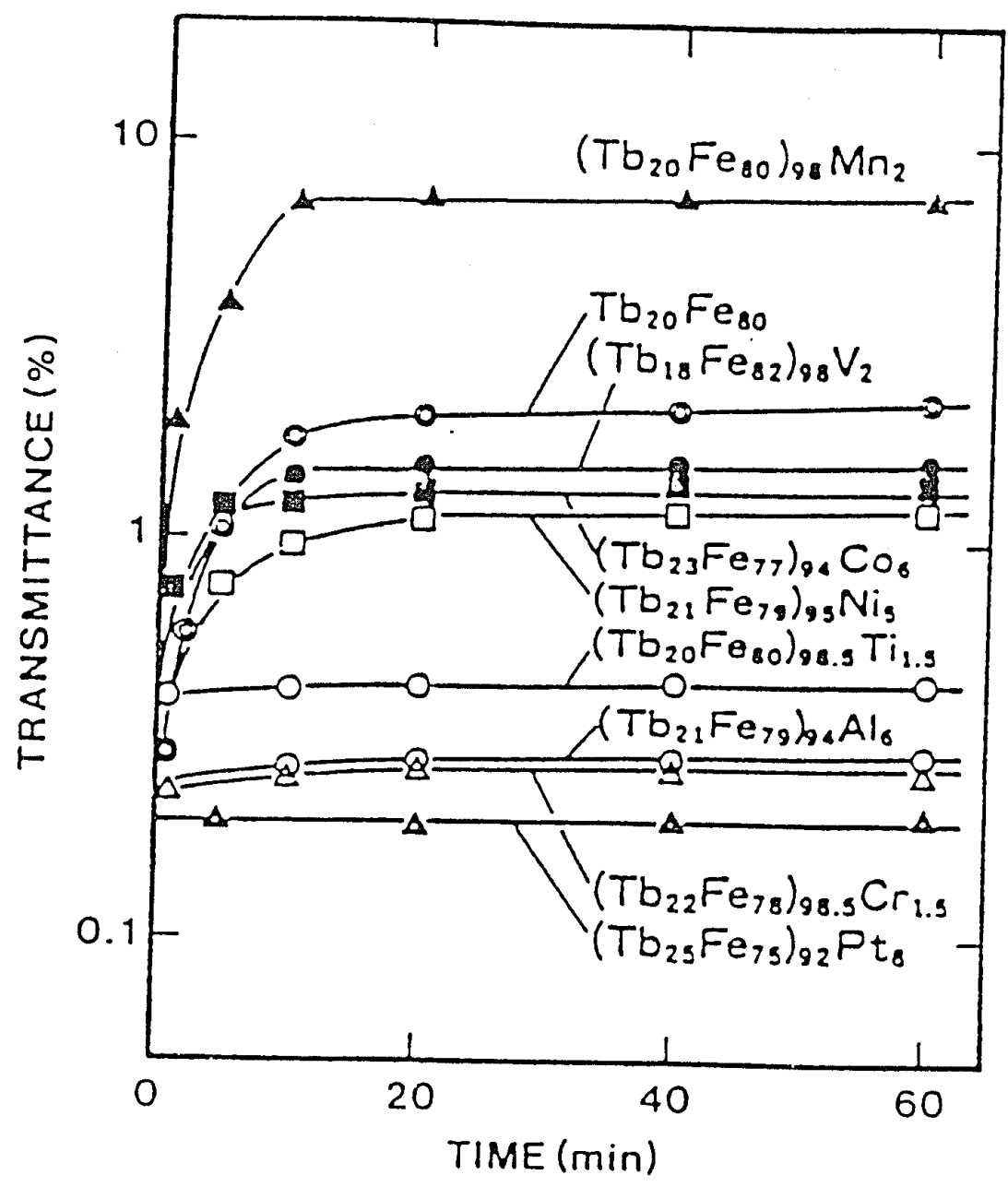

Figure 14. Effect of various alloying elements on corrosion and pinhole formation, as measured by film reflectance. (49) 
media.(3,33) Overcoats are also useful for their role in enhancing the Kerr rotation of the Mo films. 
Chapter 3

\section{EXPERIMENTAL PROCEDURE}

\subsection{Experimental plan}

The experiments for this study were designed so that the changes in magnetic and magneto-optical properties of TbFe and TbFeCo films may be studied with respect to the oxidation in these films. Furthermore, the study of structural changes and the depth of oxide formation with respect to annealing time was planned. The detailed experimental procedure is outlined in the following sections.

\subsection{Materials}

\subsubsection{Targets}

Tb and Fe targets of $99.99 \%$ purity were used for the sputtering of TbFe films. In the case of TbFeco films, Feco targets with a nominal composition of $85 \% \mathrm{Fe}$ and $15 \%$ Co were used. Silicon targets with $99.99 \%$ purity were used for the reactive sputtering of siN trilayer film structures, $40 \mathrm{~nm}$ SiN/ 100nm TbFe/ $40 \mathrm{~nm}$ SiN/ substrate. 


\subsubsection{Substrates}

The films were sputtered on clean Corning 7059 glass (1 inch $x 1$ inch), and $s i$ wafers (1 inch diameter). The substrates were cleaned by dipping in sulfuric acid, followed by cleaning in de-ionized water until the water resistivity was above 8 megohms. Subsequently, the substrates were cleaned in a vapor degreaser before loading them for sputtering. The films on glass substrates were prepared for magnetic and Kerr measurements. ESCA and TEM measurements were performed on the films deposited on the Si substrates.

\subsection{Sample Preparation Procedures}

\subsubsection{Sample Processing}

An IBM built sputtering system was used to prepare the samples for this study. The system was capable of holding four different water cooled targets. Three targets were used for DC magnetron sputtering, and one target was used for RF sputtering. The targets were located at $0,90,180$, and 270 degree locations in a cylindrical chamber. All the targets had shutters that could be manually operated. Using a diffusion pump, the system was pumped to a base pressure lower than $5 \times 10^{-7}$ torr. After achieving the base pressures, 
high purity Argon was introduced into the system with a gas flow rate of $50 \mathrm{SCCM}$, and a sputtering pressure of $5 \times 10^{-3}$ torr was achieved. The TbFe and TbFeco films were prepared using $\mathrm{DC}$ magnetron cosputtering of $\mathrm{Tb}$ and $\mathrm{Fe}$ targets, and $\mathrm{Tb}$ and Feco targets respectively. SiN protective layer films were prepared by RF sputtering of $S i$ with gas flow rates of 50 SCCM Argon and $10 \mathrm{SCCM}$ Nitrogen. The substrates were mounted on a substrate holder rotating at $30 \mathrm{rpm}$ above the targets. The MO films were deposited at a rate of about $120 \AA$ per minute.

Inficon quartz crystal oscillators were used to monitor the film thickness and composition. A relationship was established between the frequency shift readings from the quartz crystal oscillators and the film thickness readings obtained from a step height profilometer, sloan Dektak II. The Dektak measures the film thickness employing a mechanical stylus traveling over the areas on the substrate and the film, and detecting the change in the height of the stylus tip. The mass of the film is calculated from the area of the film, the film thickness, and the theoretical density of the material. This information was subsequently used to establish a relation between the readings on the quartz crystal oscillator and the number of atoms of the material reaching the substrate.

From the above correlation, it is relatively easy to control the atomic percent of the individual components in 
the film, thereby obtaining the desired film composition by varying the deposition power (current). The same information was used to obtain the final film thickness of the alloy film. It was assumed that the final film thickness is the sum of the film thicknesses that would be obtained individually from each constituent material. For the purposes of this experiment, samples with a nominal film thickness of $1000 \AA$ were deposited. The TbFe films had a nominal film composition of 20 ats $\mathrm{Tb}$ and 80 atg Fe. The TbFeCo films had a nominal composition of about 21 ato $\mathrm{Tb}, 67$ atq $\mathrm{Fe}$, and 12 atq $C o$ as measured by the $\mathrm{X}$-ray fluorescence technique.

\subsubsection{Sample Treatments}

The samples were tested in the as-deposited condition, and after oxidation in a temperature controlled oven at different temperatures and time intervals. The temperature control was measured to be within $\pm 10 \%$. The relative humidity in the oven was not controlled but was measured to be about $10 \%$. The samples were placed horizontally with the film sides up. The samples of the same material were introduced in the oven at the same time and were taken out after different oxidation times as planned. 


\subsection{Testing and Film Characterization Methods}

Various analytical techniques were employed to obtain the desired data on the samples for this study. The nominal film composition was determined using the $x$-ray fluorescence technique, and the Kerr tester was used to measure the Kerr rotation, coercivity, and reflectivity. A vibrating sample magnetometer (VSM) was used to measure the magnetization in directions parallel and normal to the film plane. The oxide depth measurements were obtained using electron spectroscopy for chemical analysis (ESCA), and a transmission electron microscope (TEM) was used to characterize the film structure.

\subsubsection{Kerr Tester}

The principle of Kerr rotation of a plane polarized light has already been discussed. The tester used to measure the Kerr rotation of the test samples is shown in Figure 15 . The Kerr tester was built at IBM Corporation and was based upon the differential detection method. The samples were held to the sample holder by vacuum. The sample holder was placed between the two poles of an electromagnet capable of providing an 8 kOe field. As shown in the figure, a linearly polarized light beam was incident upon the sample. Upon reflection from the sample, the beam was transmitted through a Wollaston polarizing beam splitter. The Wollaston prism 


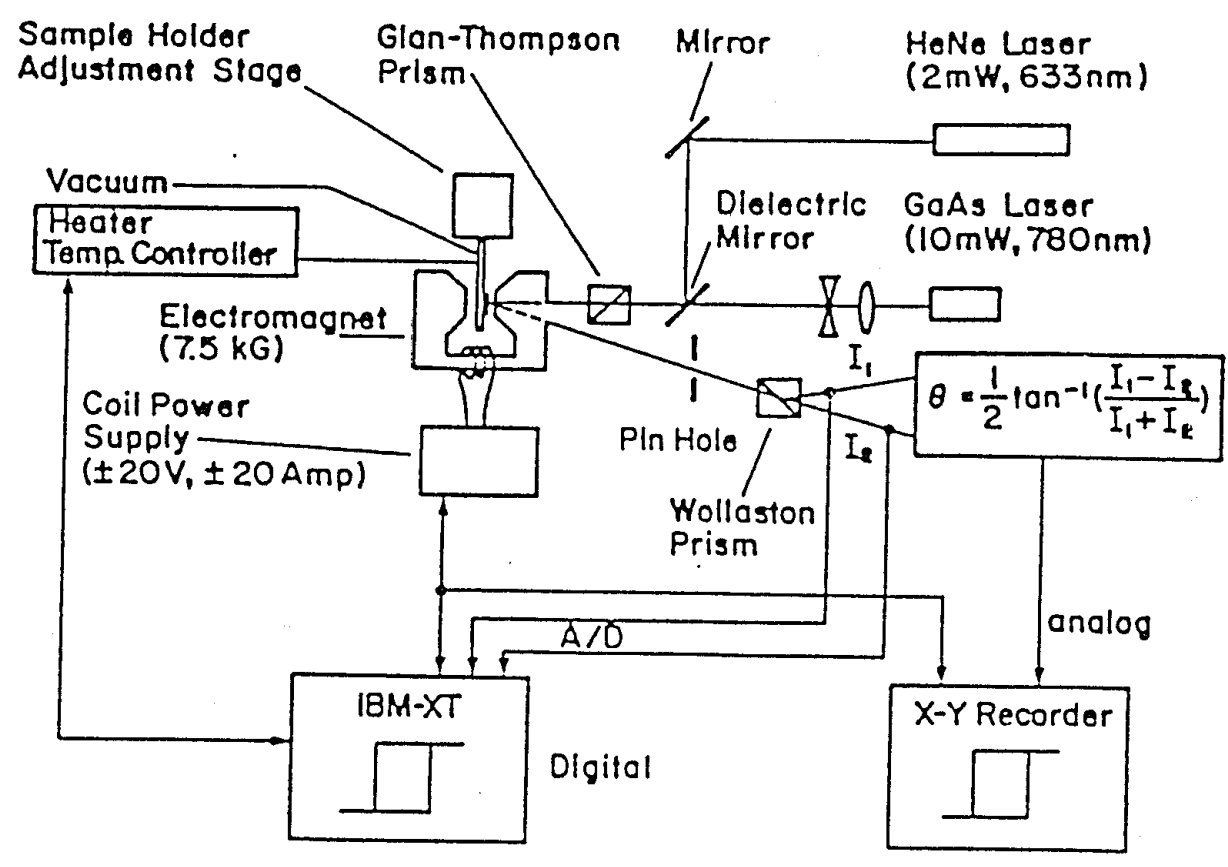

Figure 15. Schematics of the Kerr tester used for Kerr rotation and coercivity measurements. (25) 
split the rotated light into two orthogonal components, say $I_{1}$ and $I_{2}$, which were sensed by two photodetectors set to the same gain. By knowing the intensity of the two beams, the Kerr rotation angle with a repeatability of better than 1 minute was obtained from the following relationship:

$$
\theta_{k}=\frac{1}{2}\left[\tan ^{-1} \frac{\left(I_{1}-I_{2}\right)}{\left(I_{1}+I_{2}\right)}\right]
$$

The Kerr rotation measurements were done by selectively using a HeNe and a GaAlAs laser for measurements at $633 \mathrm{~nm}$ and $830 \mathrm{~nm}$ wavelength, respectively. An IBM series 1 computer with a lab automation package was used to control the loop tracing, data acquisition, and analysis. The data was plotted at the same time on a chart recorder. It was then transferred to the VM4 system for final printing.

\subsubsection{Vibrating Sample Magnetometer (VSM)}

In the case of RE-TM films, the magnetization in the samples may be of the order of $10^{-4} \mathrm{emu}$, and requires an extremely sensitive technique for measurements. The VSM is a very versatile and sensitive instrument for magnetization measurements in thin films, especially when the magnitude of the magnetization is small. During measurements, the sample 
is made to oscillate sinusoidally in the magnetic field. The magnetization of the sample induces an electrical signal in the pickup coils as shown in Figure 16. The reference signal is provided by the transducer near the upper end of the sample rod. The DC voltage proportional to the magnetic moment but independent of the vibration amplitude and frequency is derived from the signal processing circuitry.

The VSM used for the testing was made by Princeton Applied Research and was capable of testing samples up to about $1 / 2$ inch in size at an applied field of up to 19 koe and magnetization sensitivity of 10-4 emu. A maximum field of 18 KOe was used for testing the samples for this study. The samples were mounted on the sample rod using a double sided tape. The film plane of the sample could be rotated to any angle with respect to the applied field. The rotation angle of the film plane was indicated on a graduated scale. Thus, the measurements of magnetic moment could be made in directions parallel and normal to the film plane.

\subsubsection{Surface Analysis by X-ray photoelectron Spectroscopy (XPS)}

Surface analysis by $\mathrm{x}$-ray photoelectron spectroscopy is more commonly known as electron spectroscopy for chemical analysis (ESCA). The analysis of a film is accomplished by irradiating the film with monoenergetic soft $x$-rays and 


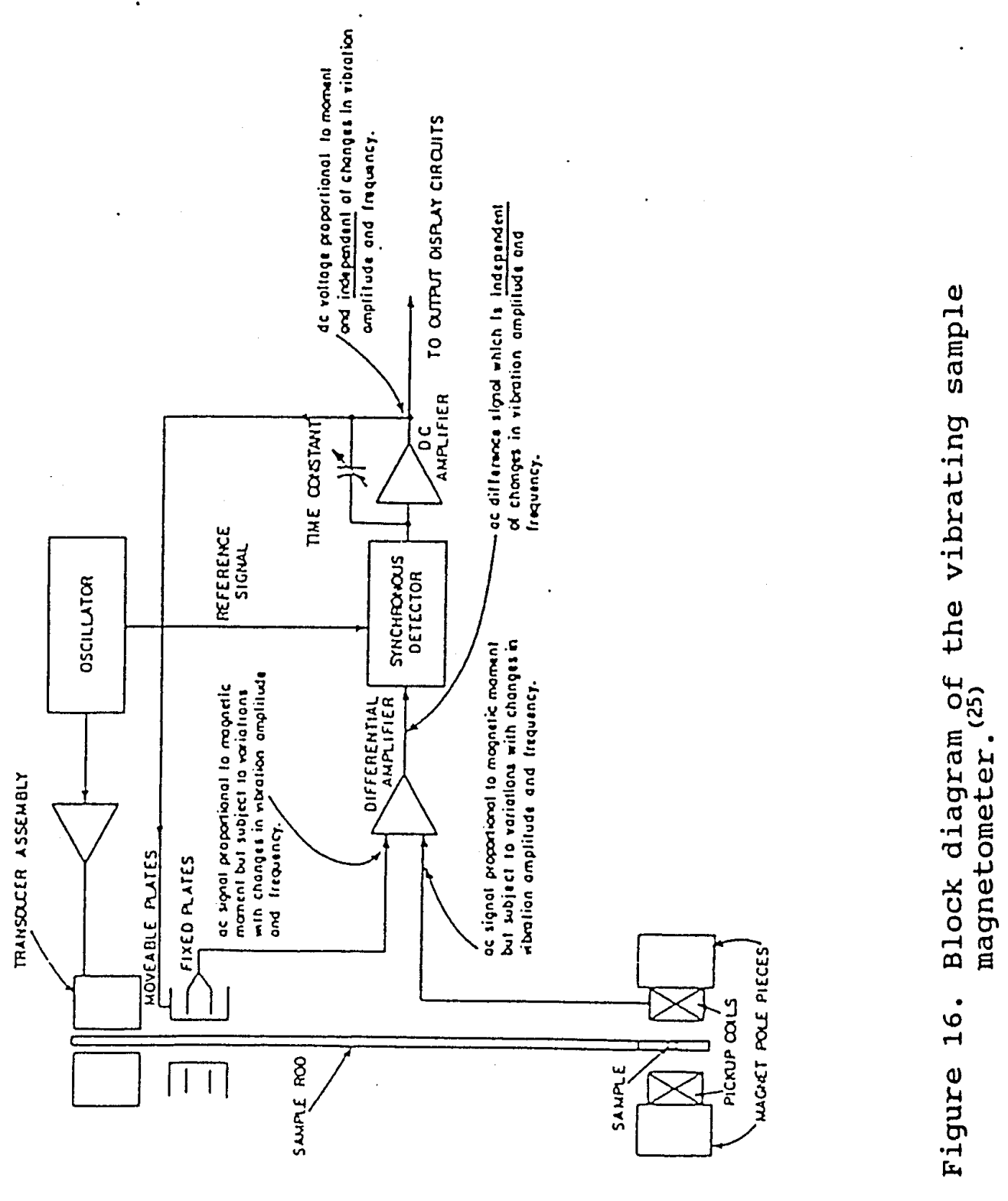


analyzing the electrons emitted. Mostly, $\mathrm{Mg} \mathrm{K}_{\alpha} \mathrm{X}$-rays $(1253.6 \mathrm{eV})$ or AI $\mathrm{K}_{\alpha}$ X-rays $(1486.6 \mathrm{eV})$ are used. They interact with the atoms in the surface region by the photoelectric effect, causing electrons to be emitted (see Figure 17). The emitted electrons have kinetic energies which are the characteristic of the material, given by

$$
\mathrm{KE}=\mathrm{hv}-\mathrm{BE}-\phi_{\mathrm{s}}
$$

where hv is the energy of the photon,

$B E$ is the binding energy of the atomic orbital from which the electron originates, and

$\phi_{s}$ is the spectrometer work function.

The binding energy may be regarded as an ionization energy of the atom for the particular shell involved. since there is a variety of possible ions from each type of atom, there are corresponding varieties of kinetic energies of emitted electrons. Since the probability of interaction of electrons with matter is high, the path length of the electrons is of the order of tens of angstroms only. Thus, only those electrons which originate within tens of angstroms below the solid surface can leave without energy loss. It is these electrons that produce the peaks in the spectra, and are most useful. The electrons leaving the sample are detected by an electron spectrometer according to their kinetic energies. Electrons are detected as discrete events, and the number of 


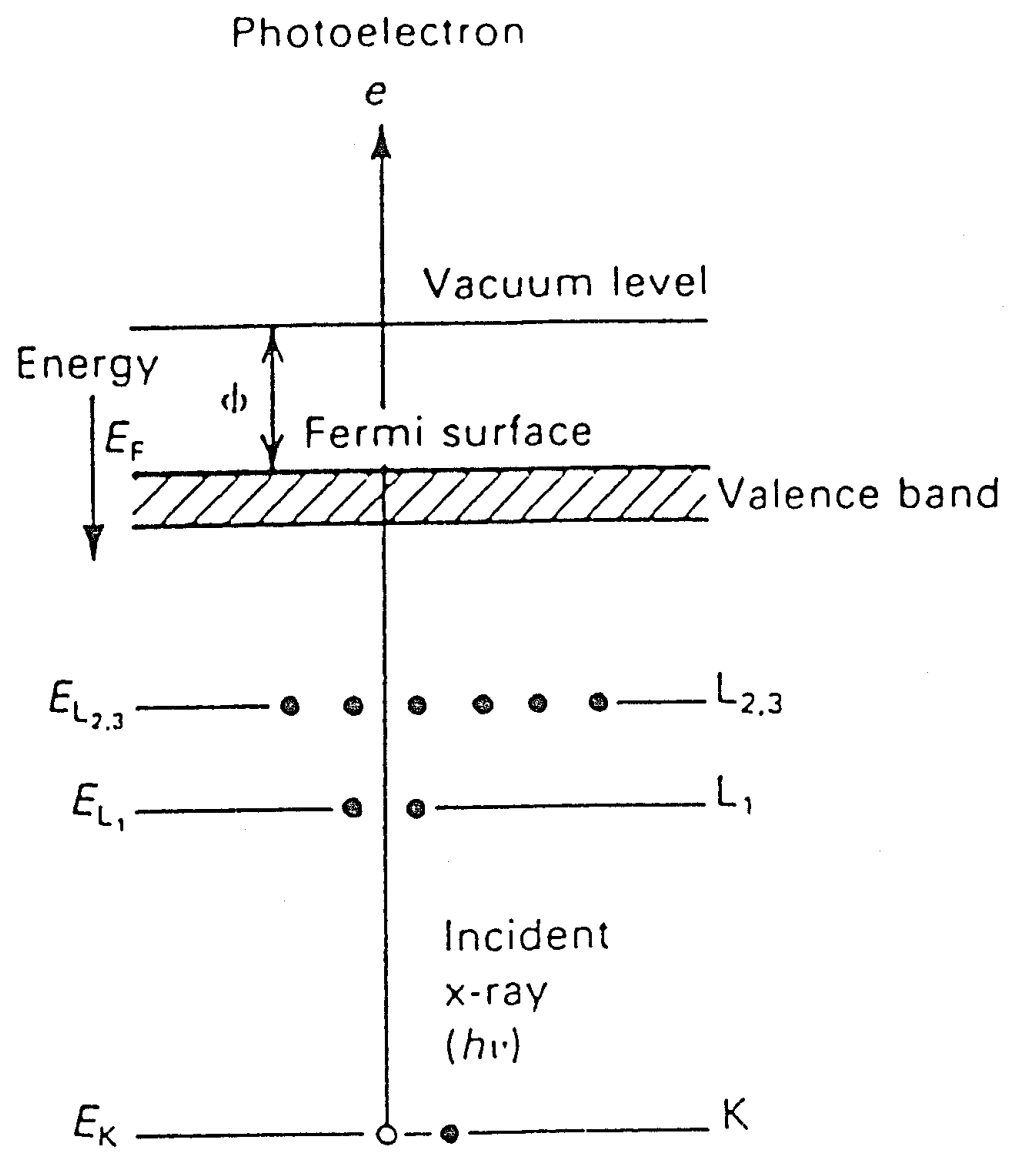

Figure 17. Energy level diagram showing the electron transition during the ESCA technique. (55) 
electrons for a given detection time and energy is either stored digitally or recorded using analog circuitry.

The oxidation depth data for the test samples in this study was obtained on a PHI 5400 Perkin Elmer spectrophotometer. Base pressures lower than $3 \times 10^{-10}$ torr were used during the analysis. For the purpose of depth profiling, sputtering was done with $3 \mathrm{KV}$ Argon ions. Sputtering rates of about $57 \AA, 27 \AA$, and $50 \AA$ per minute were measured for oxidized films of FeCo, TbO2, and Ta205. The $\mathrm{O}(1 \mathrm{~s}), \mathrm{Tb}(4 \mathrm{~d}), \mathrm{Fe}(2 \mathrm{p})$, and $\mathrm{Co}(2 \mathrm{p})$ regions were monitored for the annealed TbFe and TbFeCo films. The oxygen signal was measured at the beginning and end of each data acquisition cycle.

\subsubsection{Transmission Electron Microscopy (TEM)}

Transmission electron microscopy is unique among material characterization techniques in that it enables simultaneous examination of microstructural features and acquisition of chemical and crystallographic information from small regions of the sample. The electron ray path through a transmission electron microscope (see Figure 18) is analogous to the visible light ray path through the ground glass lenses in an optical microscope configured to operate in the transmitted light mode (except that the electrons follow a spiral path through the focusing lens). Consequently, electrons flood 


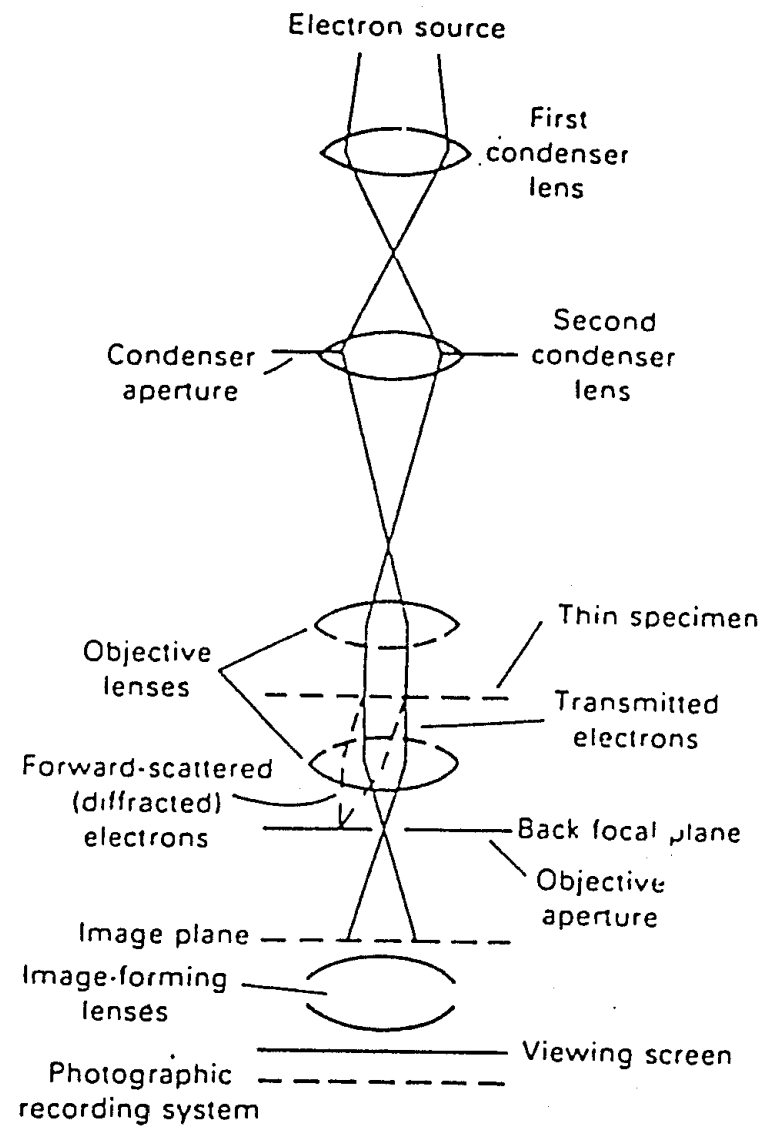

\section{Figure 18. Electron optical column in a conventional transmission electron microscope. ${ }^{(56)}$}


the sample and illuminate the area of interest. The electrons strike the thin film sample, and may undergo any of the several interactions with the sample. The electrons which undergo coherent elastic scattering in the forward direction are responsible for the formation of diffracted beams in the TEM. This results in the formation of electron diffraction patterns. Scattering angles for the electron diffraction are given by the Bragg's law:

$$
n \lambda=2 d \sin \theta
$$

where $\mathrm{n}$ is a positive integer,

$\lambda$ is the wavelength of the electrons,

d is the spacing between the diffracting planes, and $\theta$ is the diffracting angle.

Bright field (BF) and dark field (DF) are the most commonly used imaging modes for crystalline materials. The formation of both type of the images can be understood in terms of the image forming characteristics of the objective lens (Figure 19). A small aperture may be introduced in the back focal plane of the objective lens to intercept the diffracted beam, and only allow the transmitted beam to form an image. This situation is shown in Figure 19b, and is known as the bright field (BF) imaging. Alternatively, the objective aperture could be displaced from the optic axis to 

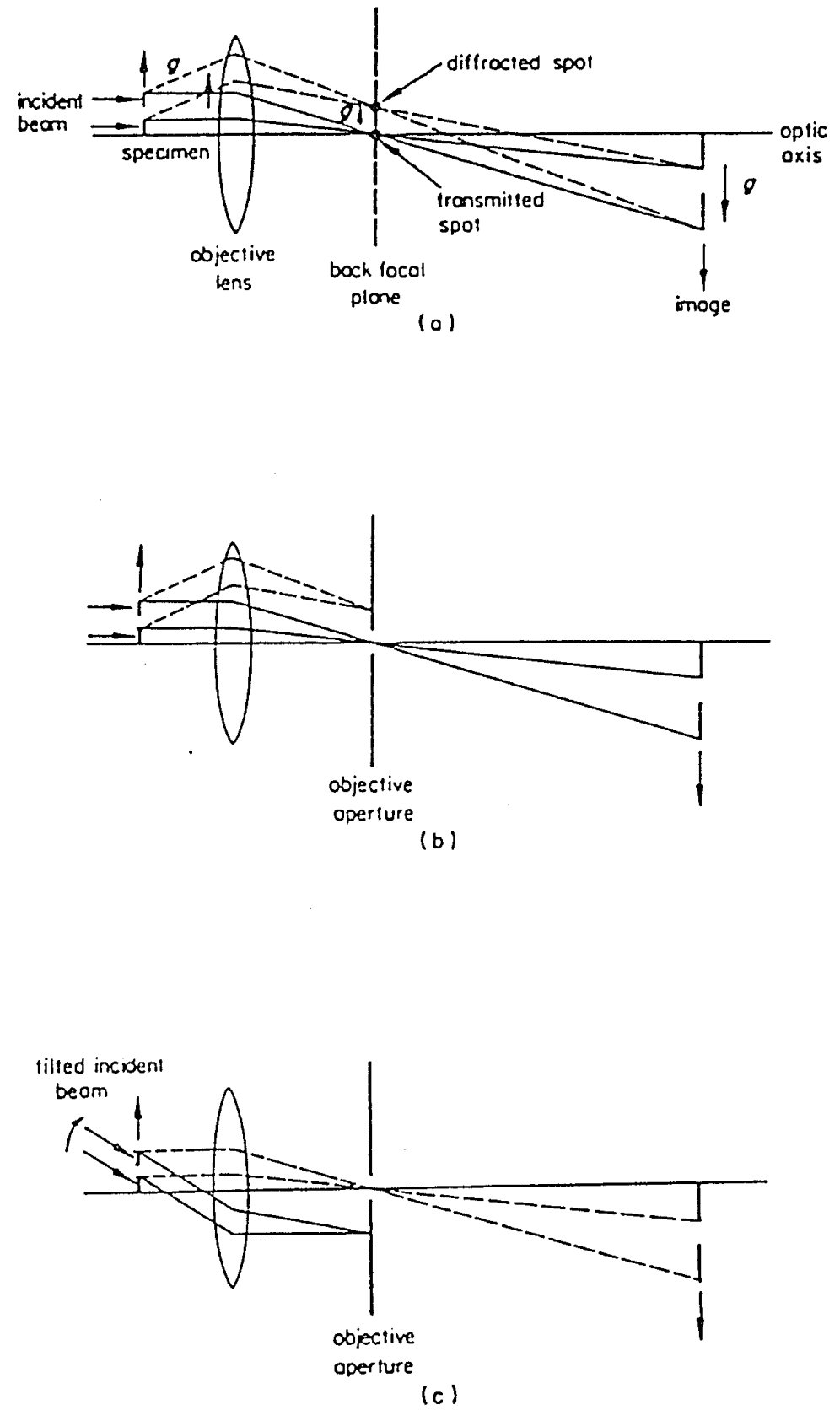

Figure 19. (a) Formation of focused diffraction pattern in the back focal plane of the objective lens, (b) position of aperture to form a BF image, and (c) tilted illumination to form a CDF image. (57) 
intercept the transmitted beam and allow the diffracted beam to contribute to the image. This is known as the dark field (DF) imaging. However, in order to retain the resolution of the BF mode, the illumination incident on the sample is tilted so that the diffracted electrons travel along the optic axis (Figure 19c). This technique is known as the centered dark field (CDF) imaging. 
Chapter 4

\section{RESULTB AND DISCUB8ION}

The results obtained during this study will be discussed in terms of the effect of oxidation on the Kerr rotation angle and coercivity changes in the film. Thereupon, the development of a surface layer with the easy axis along the film plane will be explained in terms of the changes in Kerr rotation angle and shape of the Kerr loop. The effect of the surface layer on the growth of in-plane component of magnetic moment as measured by VSM will also be discussed. The changes in the oxidation depth of the film will be discussed with respect to the oxidation time. This will be followed by TEM diffraction patterns, and bright and dark film images of as-deposited and oxidized areas of the MO films. Most of the data from this study on TbFeco films has already been published. ${ }^{(53)}$

\subsection{Effect of oxidation on Rerr Rotation and coercivity}

The magneto-optical hysteresis loops as a function of annealing time at $70^{\circ} \mathrm{C}$ are shown in Figures 20 and 21 for TbFe and TbFeco films respectively. It can be seen from these figures that the Kerr rotation angle of the TbFeco films is higher than that of the TbFe films. The Kerr 

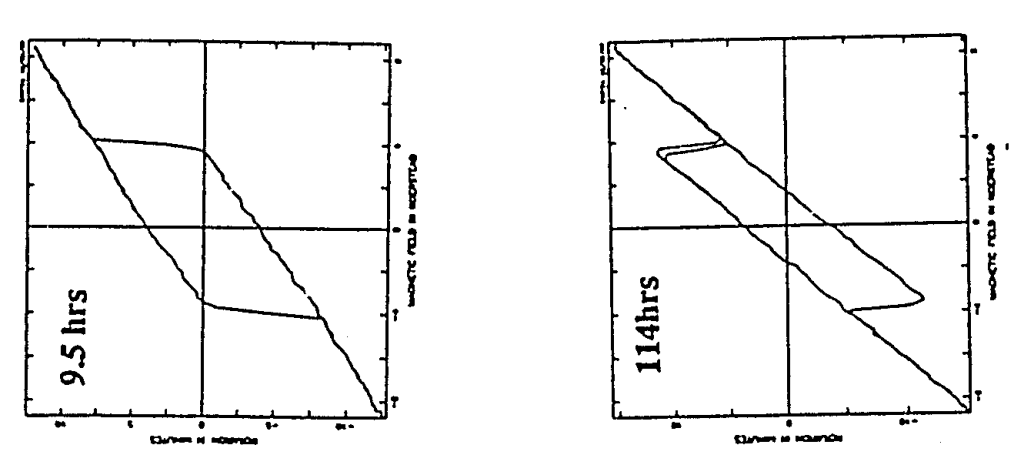

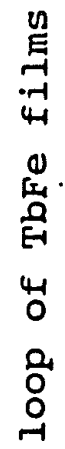
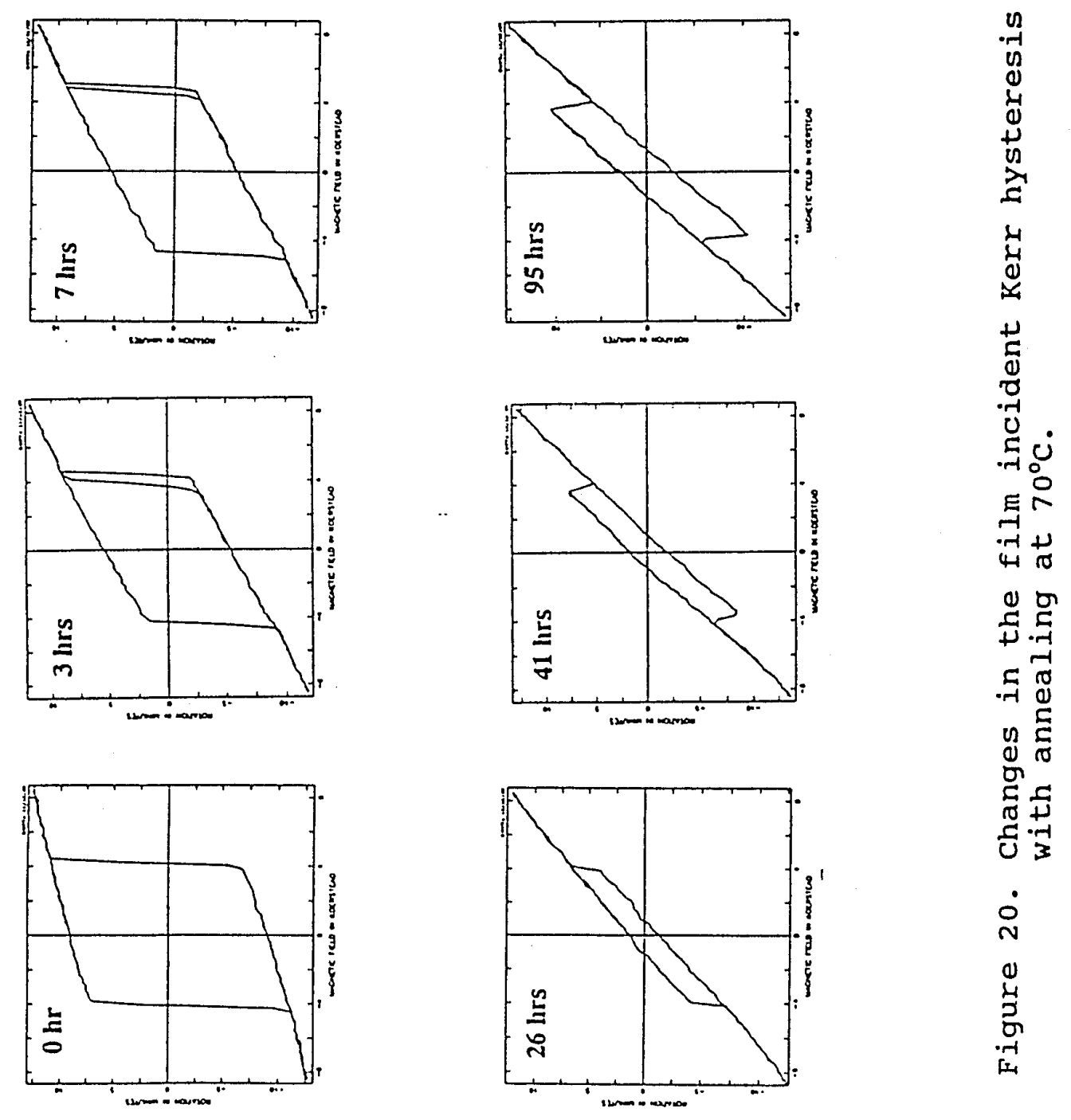

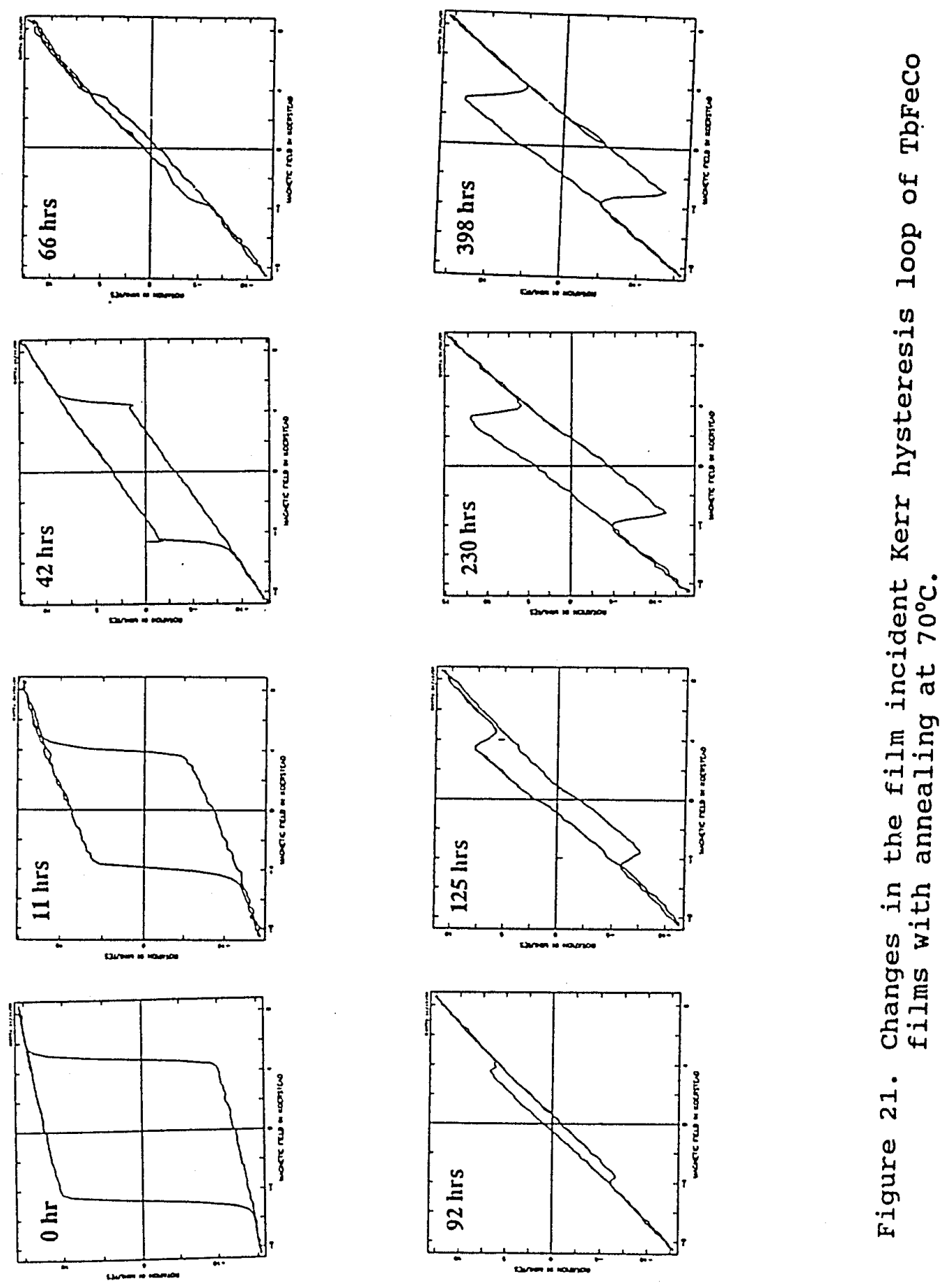
rotation angle for these films, measured with light incident from the film side, continuously decreases with annealing. In the as-deposited condition, the TbFe and TbFeCo films have their easy axes normal to the film plane. Therefore, the slanting loops for non-annealed samples indicate the presence of a hard axis magnetic component in the film, i.e., a layer with its easy axis parallel to the film plane. The slope of the curve between Kerr rotation angle and applied magnetic field increases with the annealing time. For TbFe as well as TbFeco films, the Kerr rotation angle decreases to zero before the Kerr loop begins to develop again with an inverted loop sense. However, it is noteworthy that the inversion of loop sense occurs much earlier (about 30-35 hours) in TbFe films than in TbFeco films (at about 80 hours anneal time). This indicates the higher kinetics of changes in the properties of TbFe films as compared to those of TbFeco films. Both type of films also exhibit a decrease in the film coercivity. The Kerr loops measured from the substrate side (through glass) do not indicate a significant change in the Kerr rotation angle. The coercivity changes are similar to those measured from the film side. The Kerr loops measured from the substrate side are shown in Figure 22 for TbFeco films. The film incident Kerr rotation and coercivity, normalized with respect to non-annealed samples, are shown in Figure 23 and Figure 24 for $\mathrm{TbFe}$ and TbFeCo films, respectively, as a function of annealing time. The 

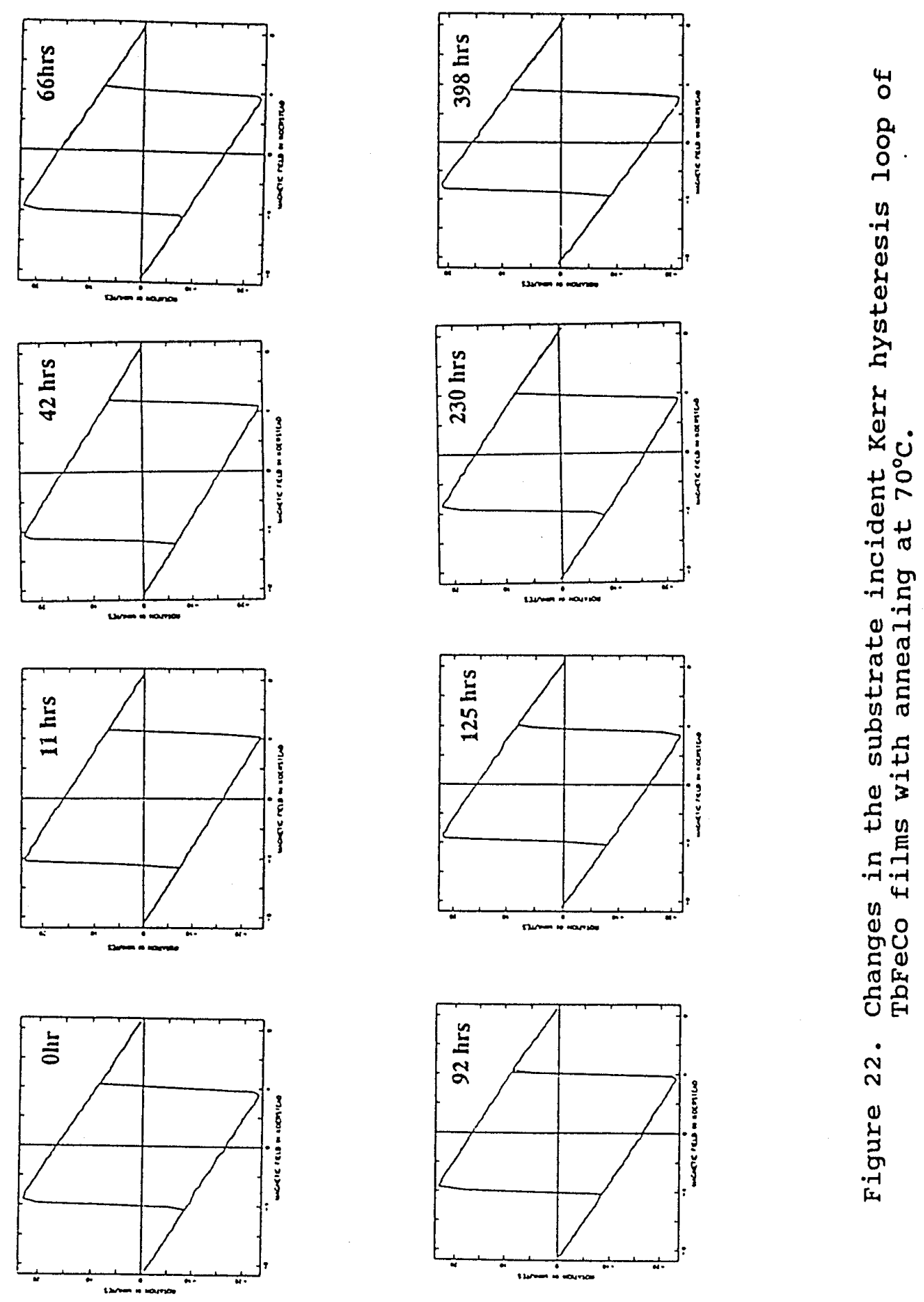


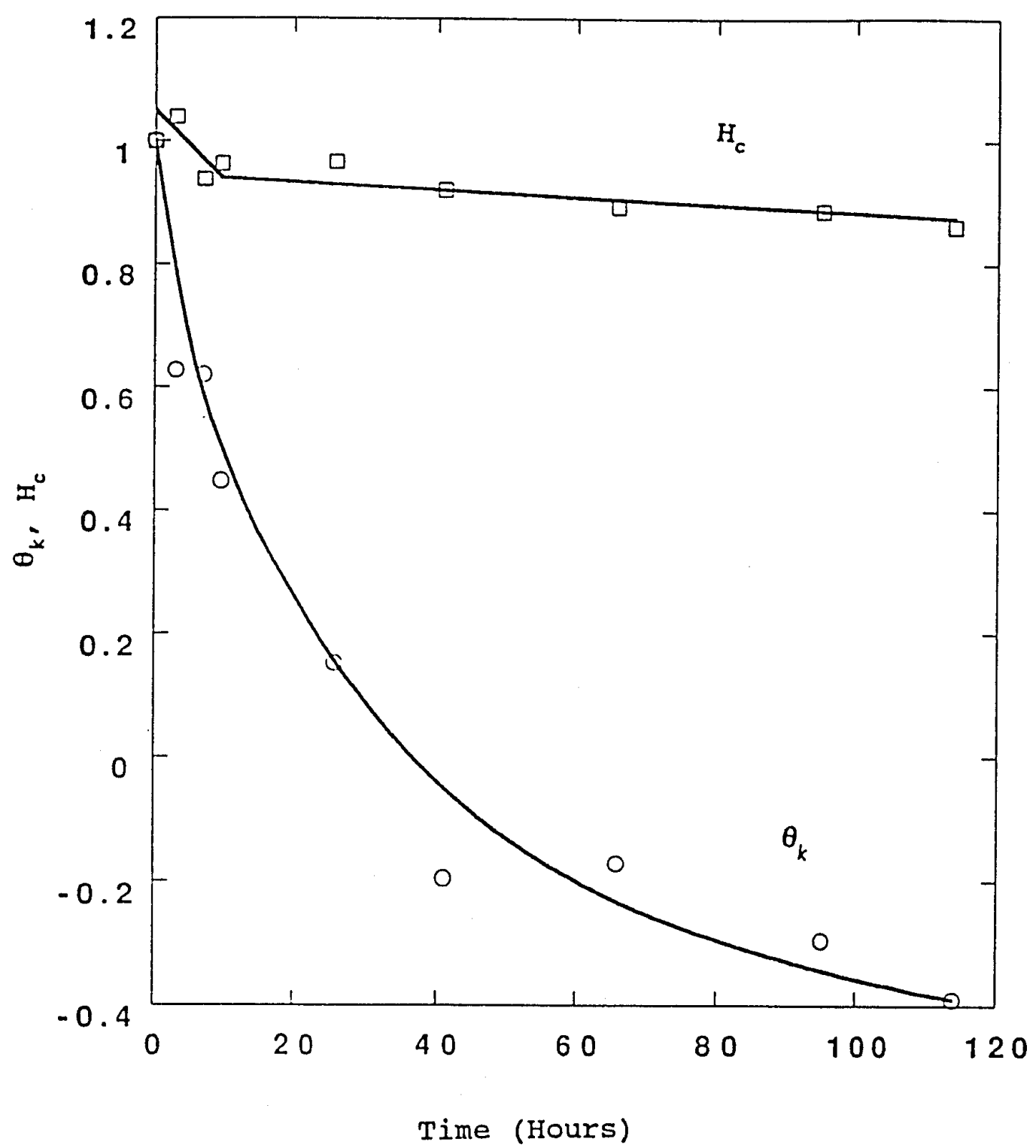

Figure 23. Normalized Kerr rotation $\left(\theta_{k}\right)$ and coercivity $\left(\mathrm{H}_{\mathrm{c}}\right)$ of $\mathrm{TbFe}$ films as a function of annealing time at $70^{\circ} \mathrm{C}$. 


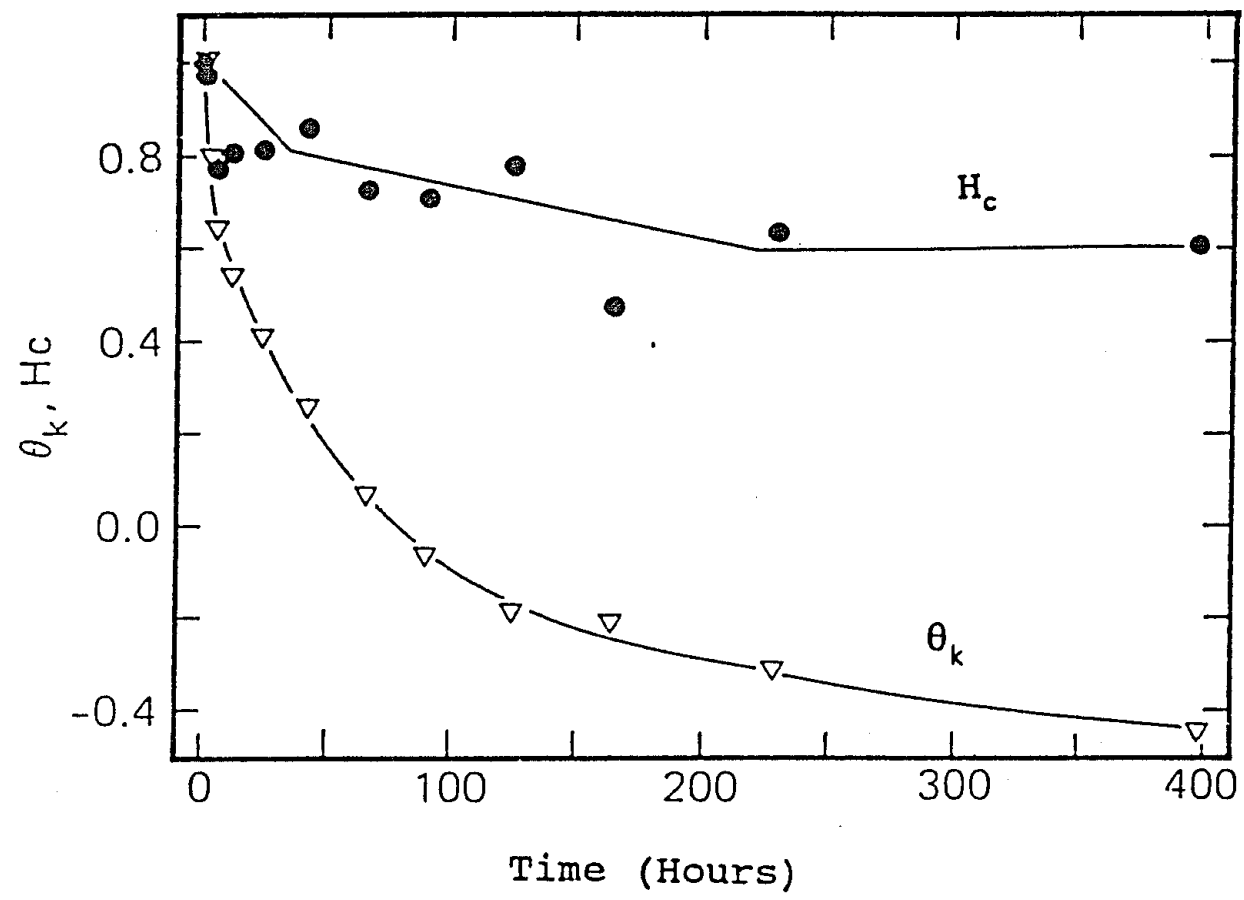

Figure 24. Normalized Kerr rotation $\left(\theta_{k}\right)$ and coercivity $\left(\mathrm{H}_{\mathrm{c}}\right)$ of TbFeCo films as a function of annealing time at $70^{\circ} \mathrm{C}$. 
Kerr rotation measured from the inverted loop is plotted as a negative Kerr rotation.

\subsection{Magnetic Measurements using VSM}

Magnetic hysteresis loops were obtained for the asdeposited and annealed samples of TbFe and TbFeCo, with the applied field direction parallel and normal to the film plane. Figures $25 \mathrm{a}$ and $25 \mathrm{~b}$ show the changes in the parallel and perpendicular components of the TbFe film. Figure 26 represents similar data for TbFeco films. These measurements clearly indicate the growth of the magnetic moment of these films, measured in the film plane direction. The normal and in-plane components of the saturation magnetization and the perpendicular coercivity of the films, normalized with respect to the non-annealed samples, are plotted for TbFeco films as a function of $t_{i m e}{ }^{1 / 2}$ in Figure 27a. The relationship between the parameters shown in the figure appears to be linear. Similarly, Figure $27 \mathrm{~b}$ depicts the growth in the coercivity and in-plane component of the magnetic moment of TbFe films. The increase in saturation magnetization of the normal component measured by the VSM (Figure 27a) also indicates the growth of the magnetic layer with the easy axis along the film plane. The negative slope of the in-plane and normal components of magnetization with applied field, as seen in Figures 25 and 26 , is due to the 


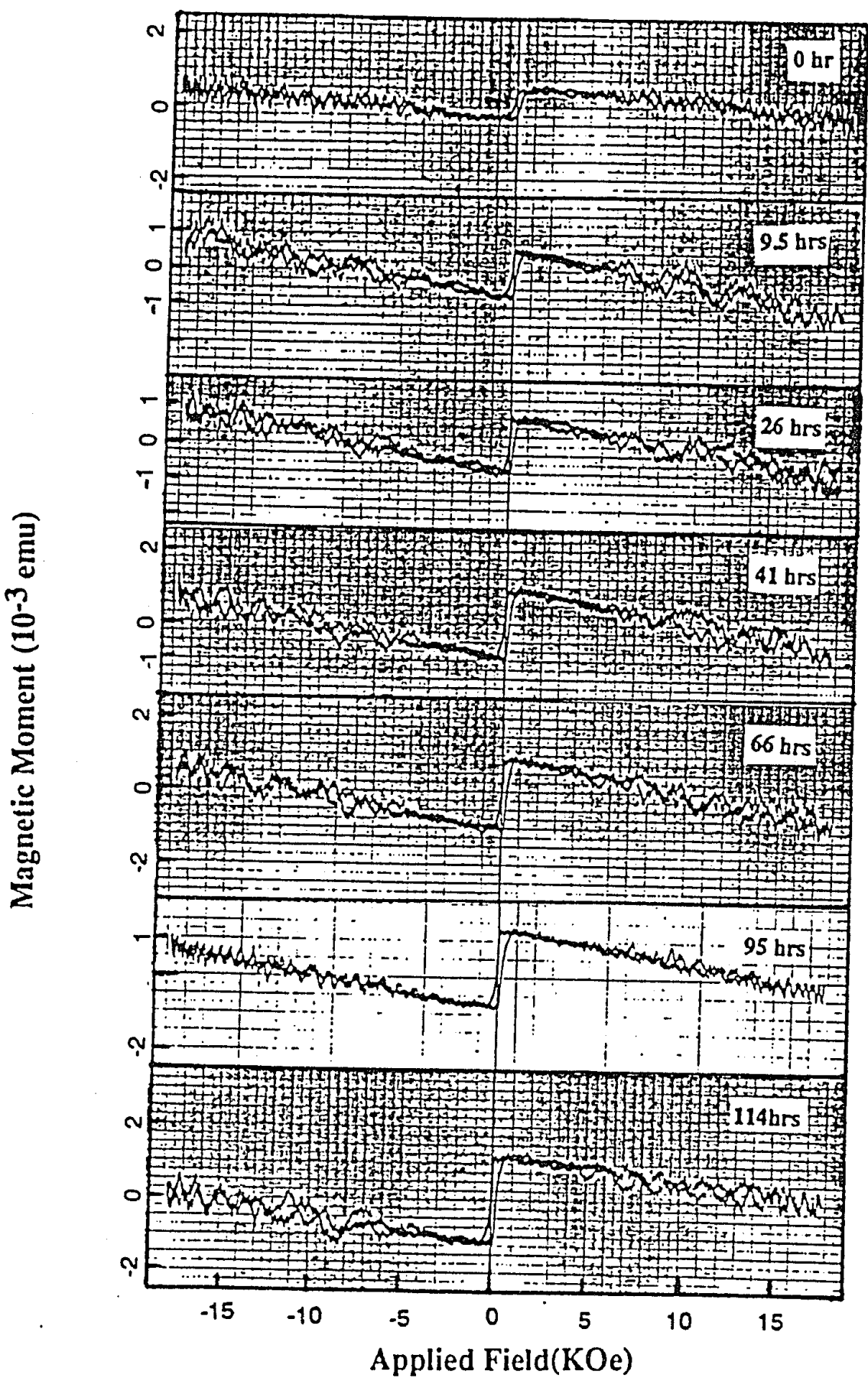

Figure 25. (a) Growth in the parallel component of magnetization of TbFe films with annealing at $70^{\circ} \mathrm{C}$. 


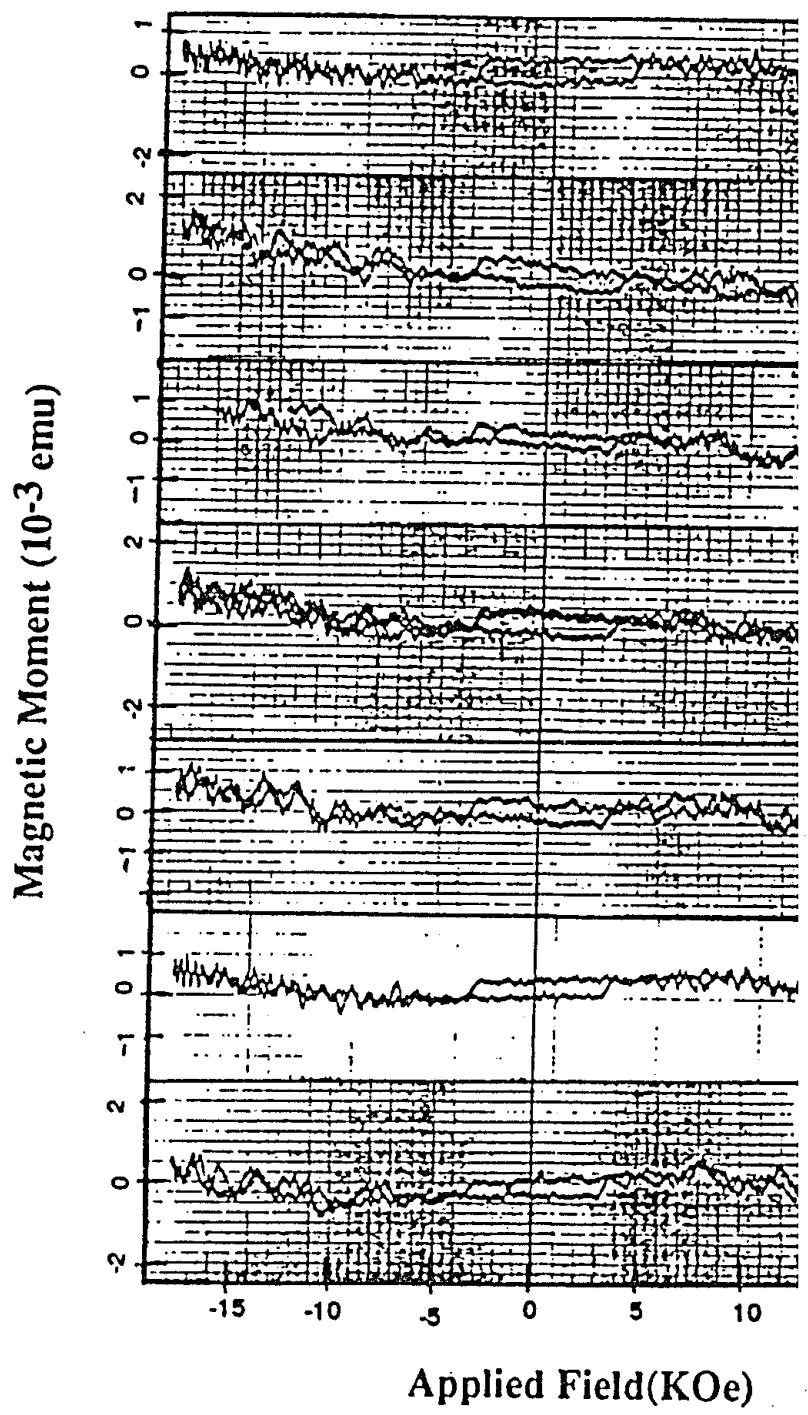

Figure 25. (b) Changes in the pular hysteresis loop of $\mathrm{Tb}$ with annealing at $70^{\circ} \mathrm{C}$. 


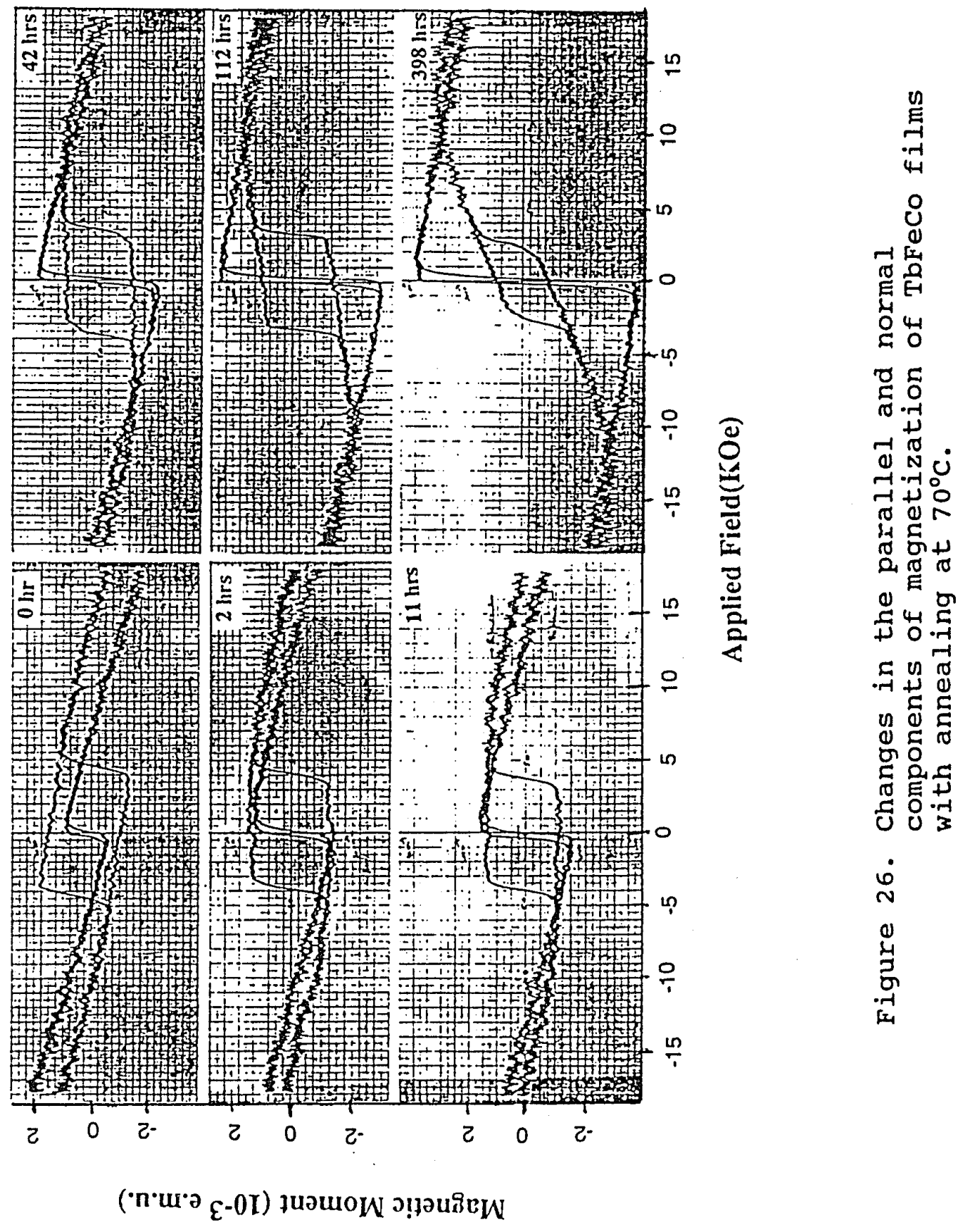




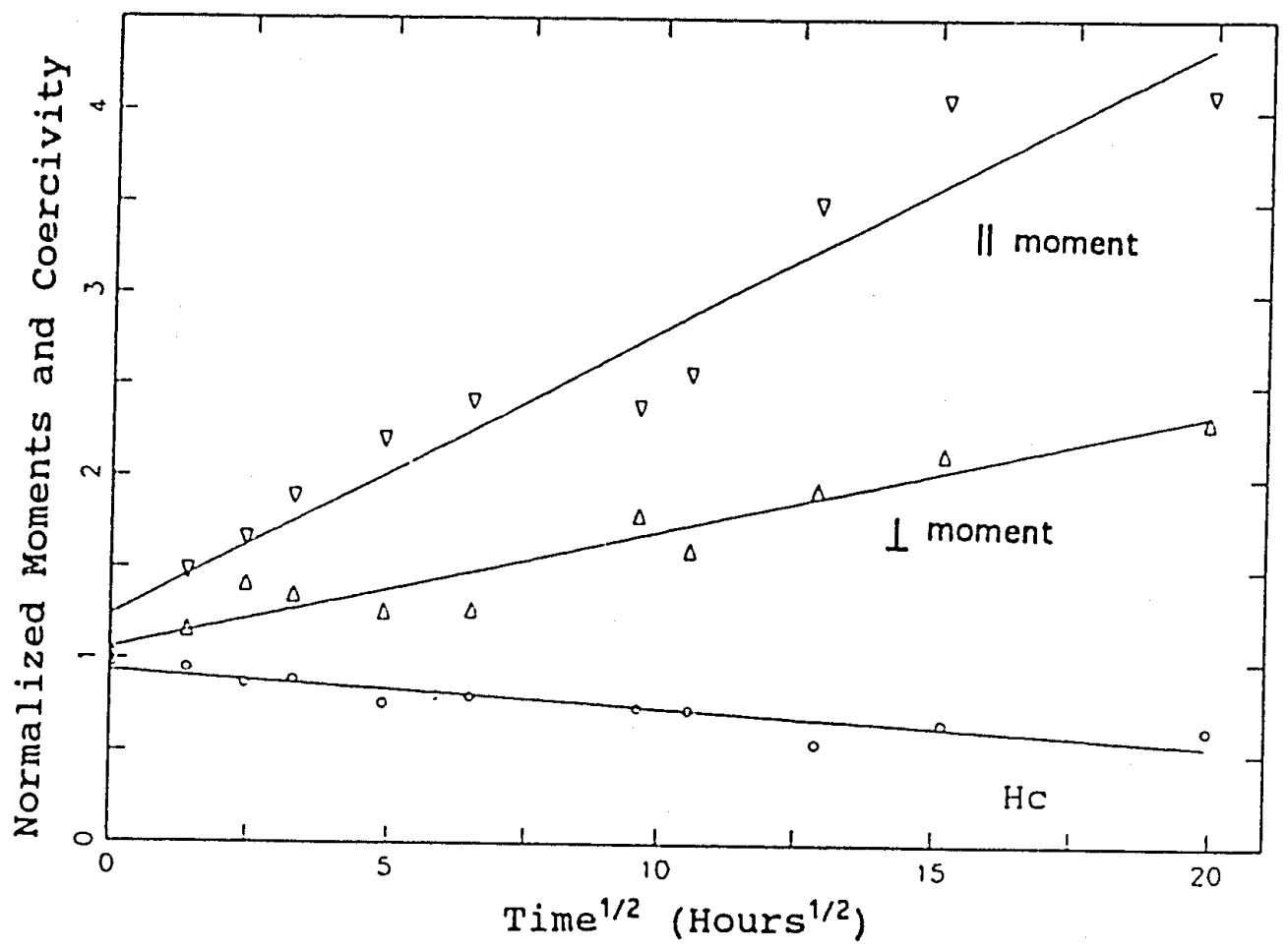

Figure 27. (a) oxidation induced changes in TbFeco films annealed at $70^{\circ} \mathrm{C}$, as a function of time $e^{1 / 2}$. 


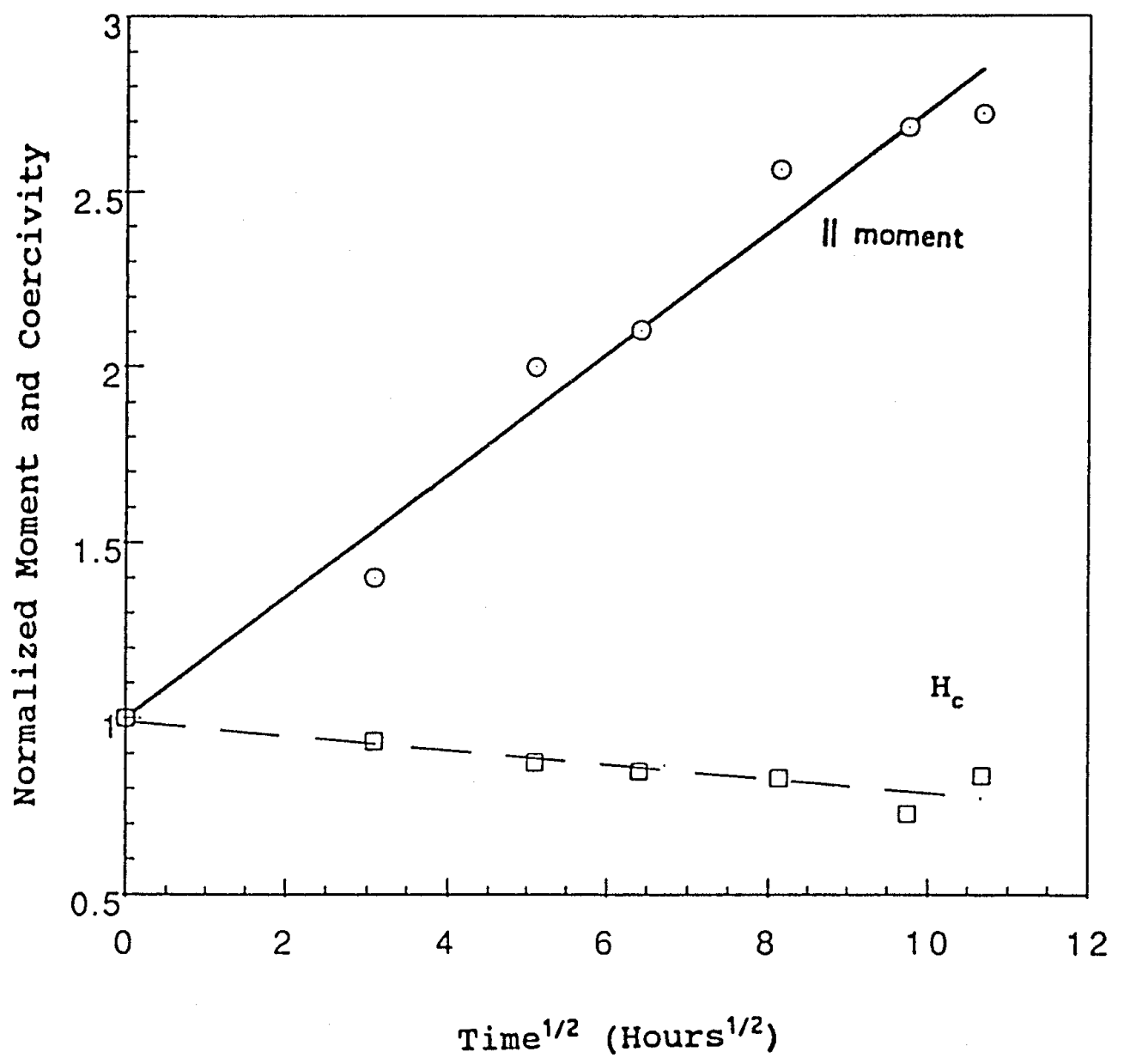

Figure 27. (b) Oxidation induced changes in TbFe films annealed at $70^{\circ} \mathrm{C}$, as a function of time $e^{1 / 2}$. 
diamagnetic behavior of the 7059 glass substrate used for preparing the test samples. This diamagnetic behavior of the substrate is shown in Figure 28, which includes the hysteresis loops for magnetic measurements performed with direction of the applied field parallel and normal to the surface of the substrate.

\subsection{Oxidation Depth versus Annealing Time}

Figures $29 \mathrm{a}$ and $29 \mathrm{~b}$ indicate the compositional changes in TbFeco samples from the film surface to the film-substrate interface as measured by the ESCA depth profiling technique. Figure 30 shows similar results, with respect to the changes in film composition, for the TbFe samples. For the nonannealed samples, these figures clearly indicate the $\mathrm{Tb}$ enrichment (with respect to $\mathrm{Fe}$ and $\mathrm{CO}$ ) at the surface of these films. As can be seen, the $\mathrm{Fe}$ to $\mathrm{Tb}$ ratios at the surface are about 0.5 and 2.0 for TbFeCo and TbFe films respectively, whereas the values of these ratios in the bulk of the film are about 3.5 and 5 , respectively. The Fe to co ratio for TbFeco films is $>15$ at the surface and about 4.5 in the bulk of the film as measured from the depth profile of the non-annealed sample. These depth profiles do not indicate the presence of an iron oxide top layer for TbFe or TbFeCo(53) films. The presence of this surface layer on $\mathrm{TbFe}$ films has been reported by other workers ${ }^{(45,46)}$ for both, as- 


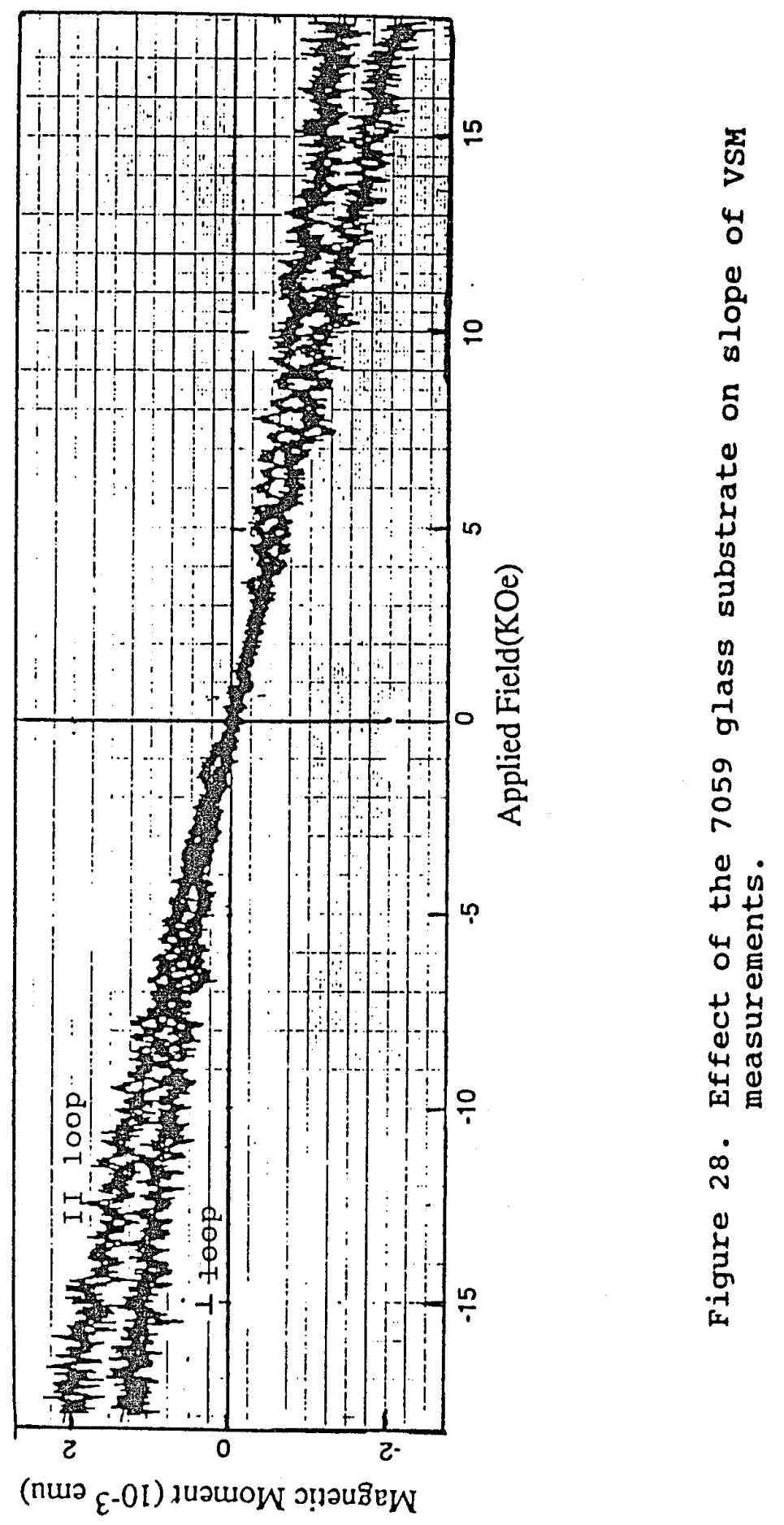



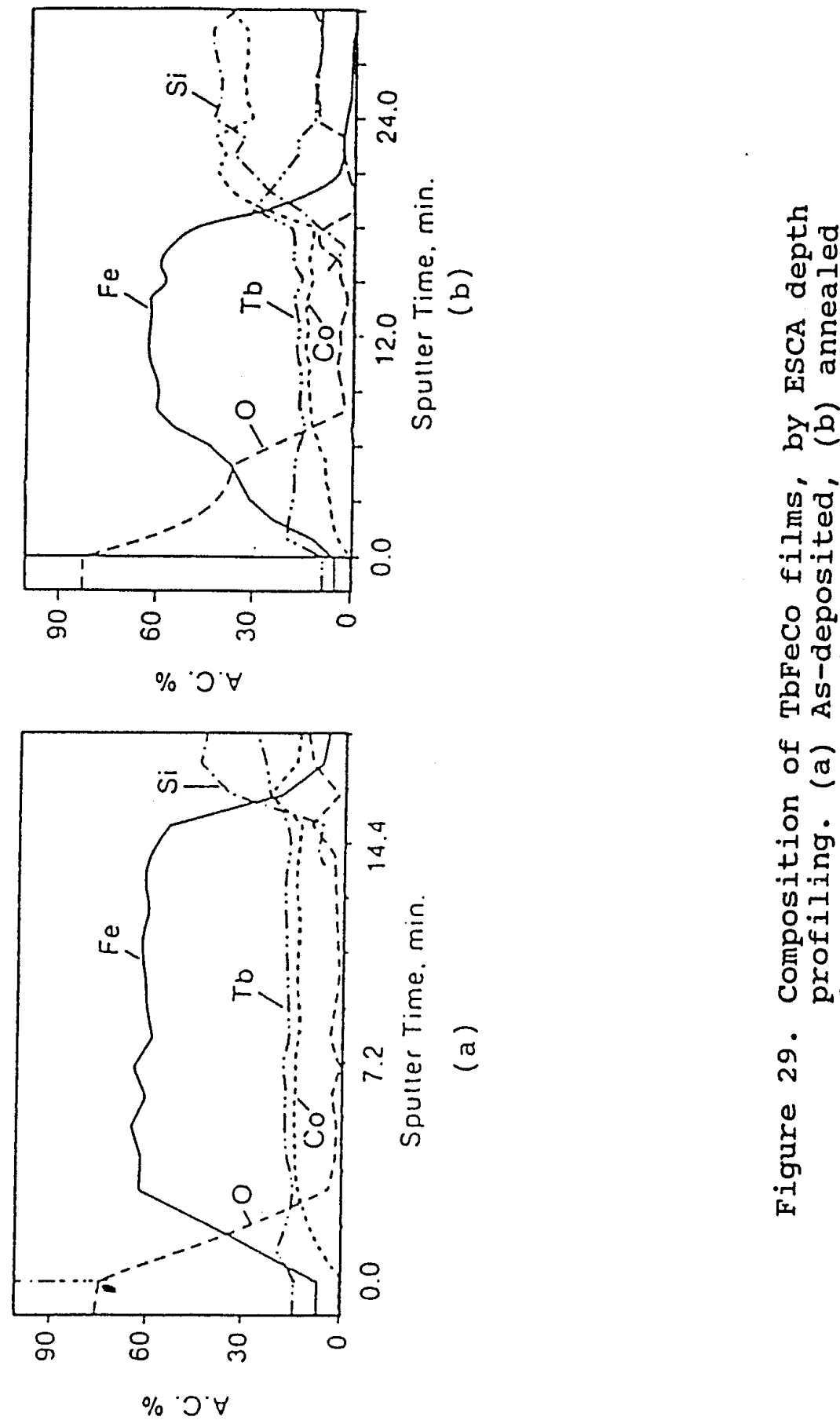

고ㅁㅗㅡ

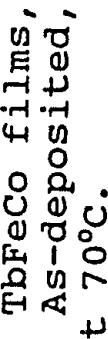

40

00

○ิ

武象오

is

$0 .+10$

a 4

텅 눙

ن눈어

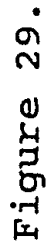

a

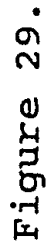



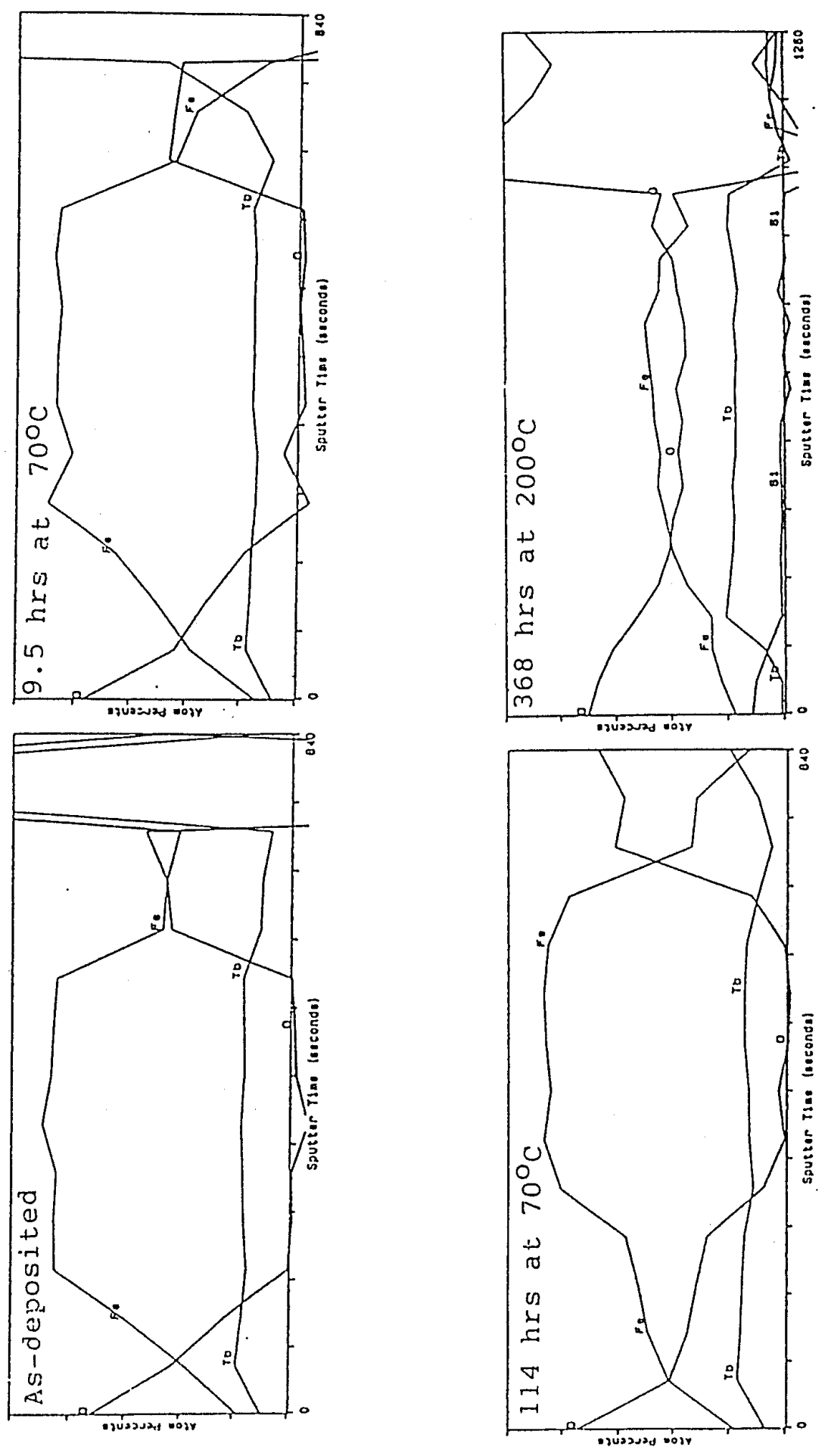

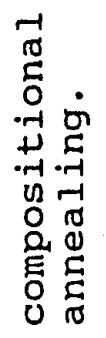

焉

o..-7

o 4

年望

얼

Q

도

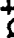

ष

它

ज合

品 
deposited samples and on samples arnealed at $200^{\circ} \mathrm{C}$ for 5 hours. However, in this study the TbFe films do contain a higher ratio of $\mathrm{Fe}$ to $\mathrm{Tb}(2.0)$ than in the case of TbFeco films $(0.5)$. In a previous work, ${ }^{(53)}$ it was proposed that the lack of $\mathrm{Fe}_{2} \mathrm{O}_{3}$ overlayer in TbFeCo samples might be due to the addition of co to the film or due to different treatments of the samples.

Figures $31 \mathrm{a}$ and $31 \mathrm{~b}$ show shifts in the binding energy for $\mathrm{Fe}_{2} \mathrm{O}_{3}-2 \mathrm{p}$ doublet as compared to the $\mathrm{Fe}$ peaks. Similarly, the shift in the Coo peaks with respect to the co metal peaks is also well characterized, ${ }^{(54)}$ as illustrated in Figures $31 \mathrm{c}$ and 31d. This enables the determination of the chemical state of Fe and Co during the ESCA depth profiling, i.e., whether Fe and/or co exist in the film in metal form or in the oxide form. Upon oxidation, binding energy profiles for the $\mathrm{Tb}-4 \mathrm{~d}$ signal change shape but do not exhibit a simple binding energy shifted oxide peak as in the case of $\mathrm{Fe}$ and Co. From the shape of the signal, a qualitative idea of the state of $\mathrm{Tb}$ in the film may be possible. In the case of the 0-1s signal, the binding energy peak shifts to higher values when oxygen exists in a hydroxide form as compared to its binding energy in an oxide form.

The binding energy peaks for TbFe films corresponding to Tb-4d, Fe-2p, and o-1s at different annealing times are shown in Figure 32. Figure 33 indicates the compositional depth profile and binding energy peaks for TbFeCo films heated for 

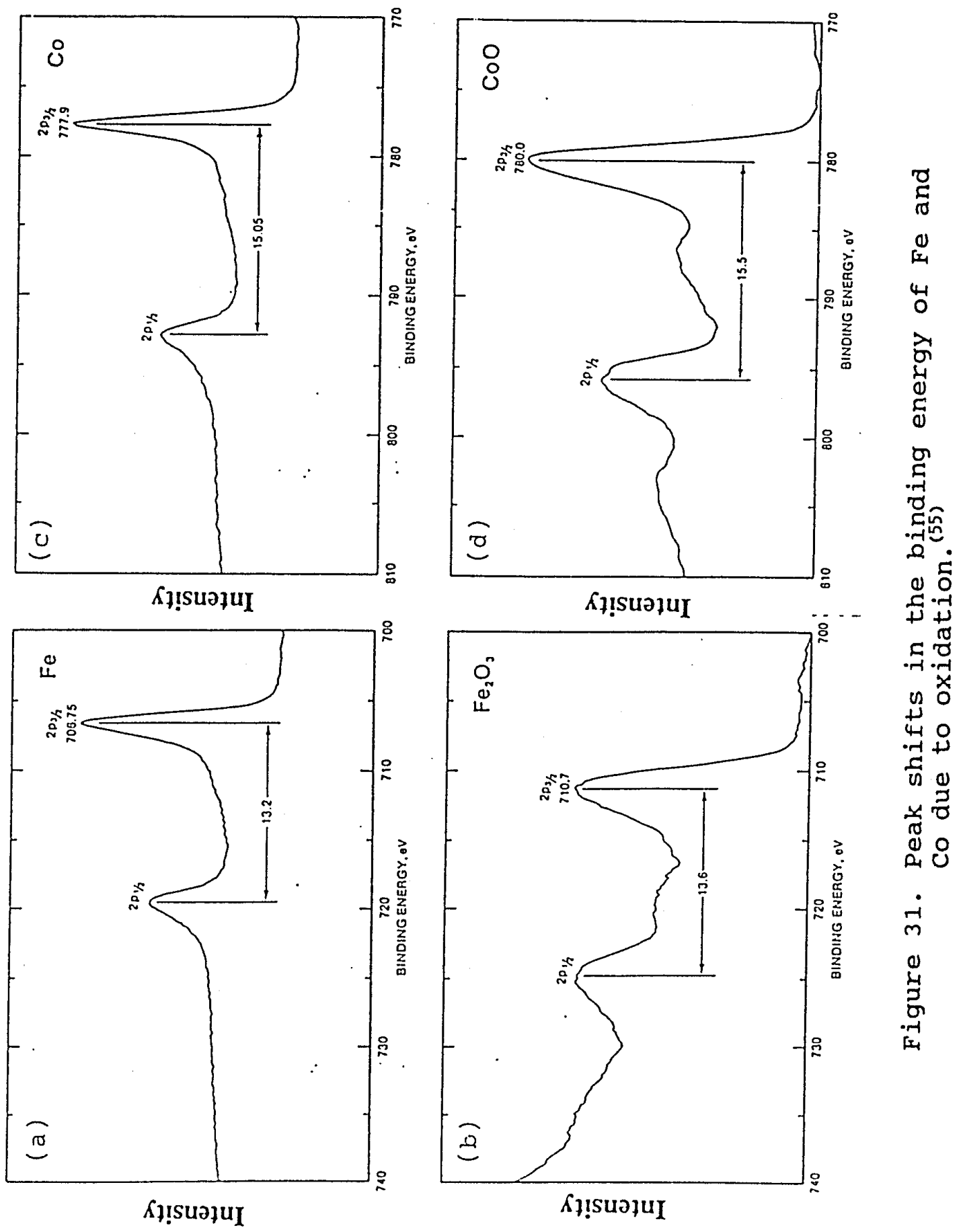

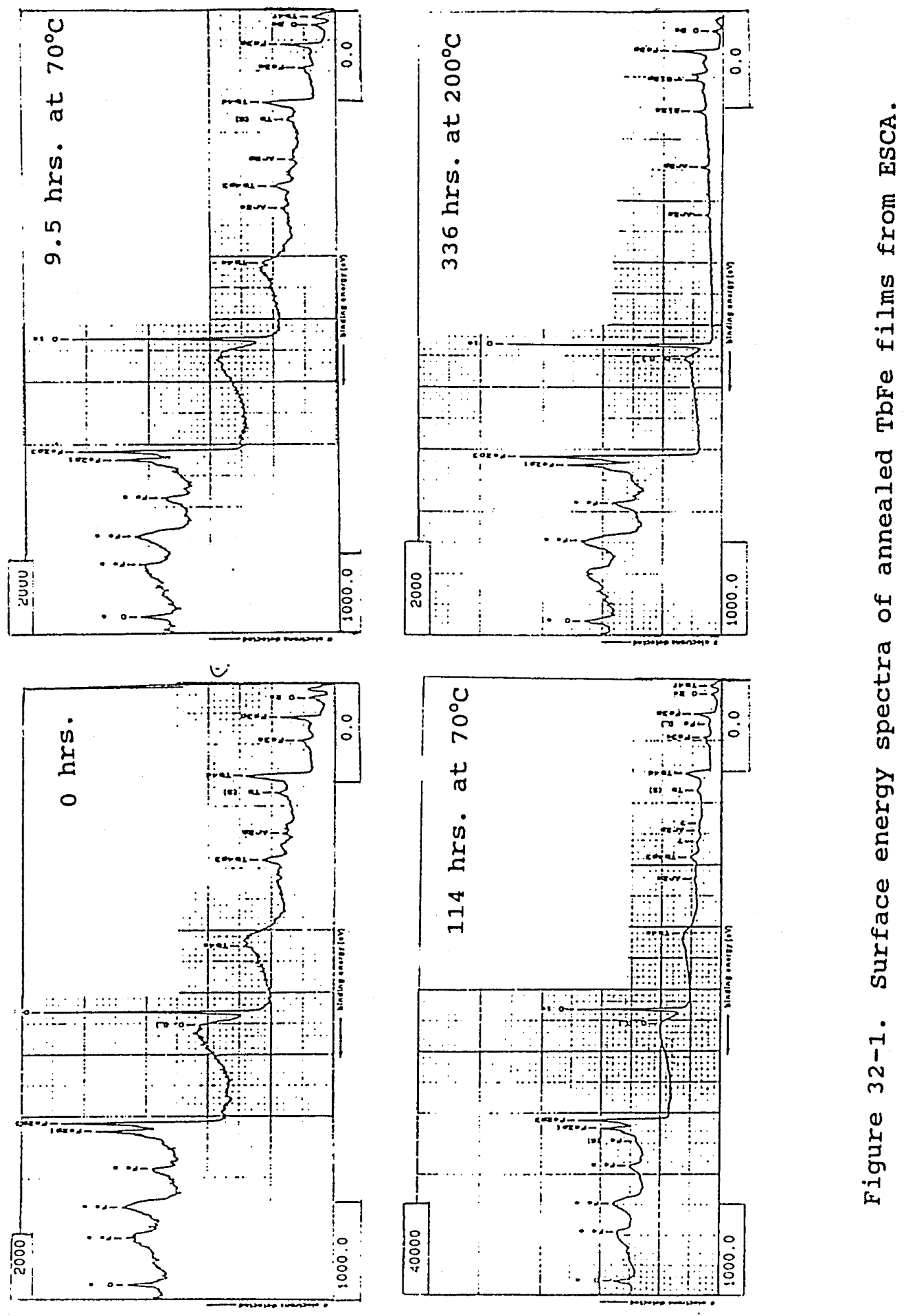

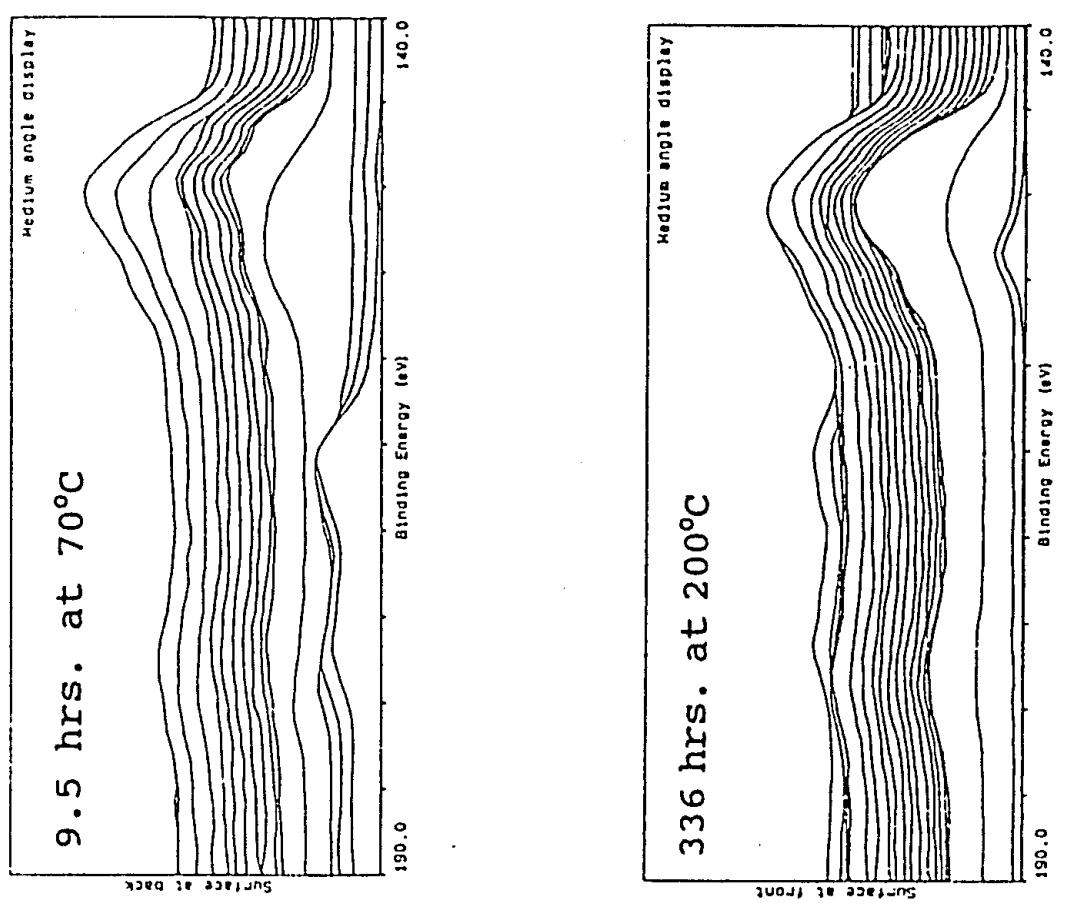

告
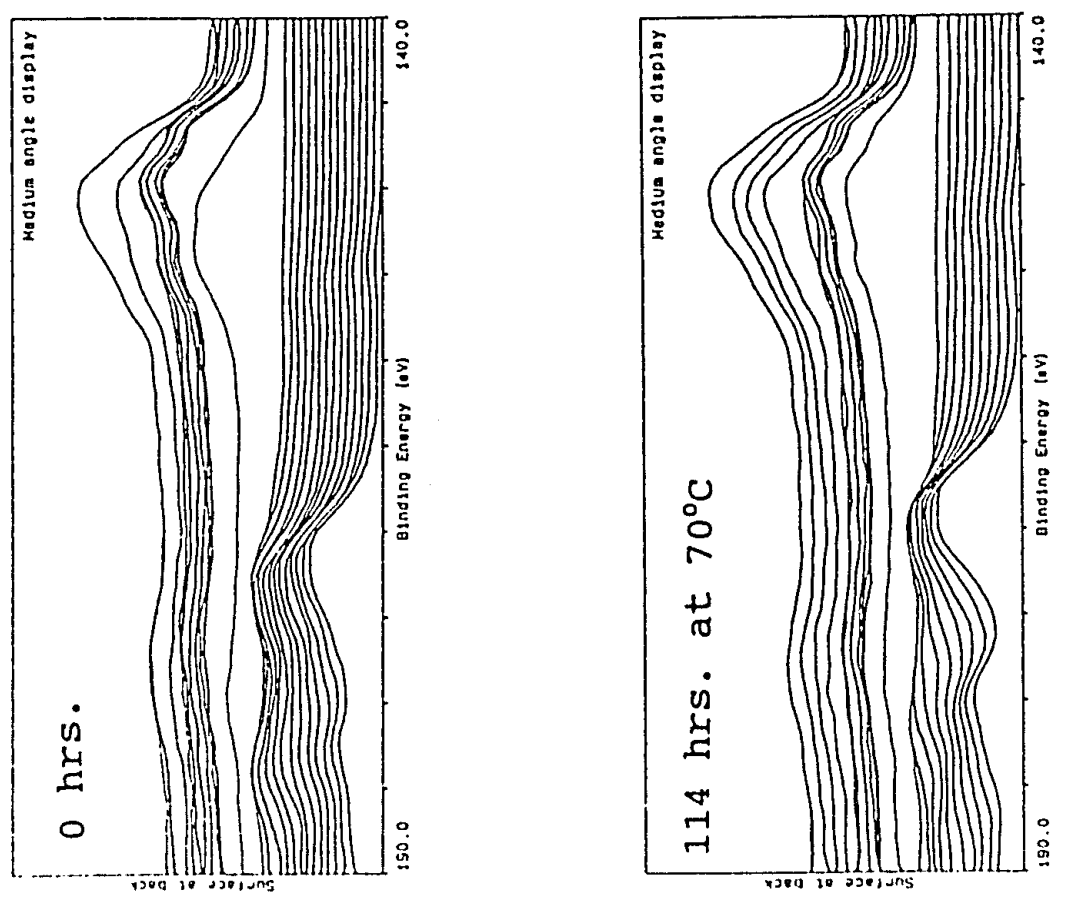

岕

最

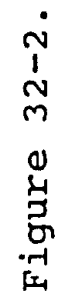



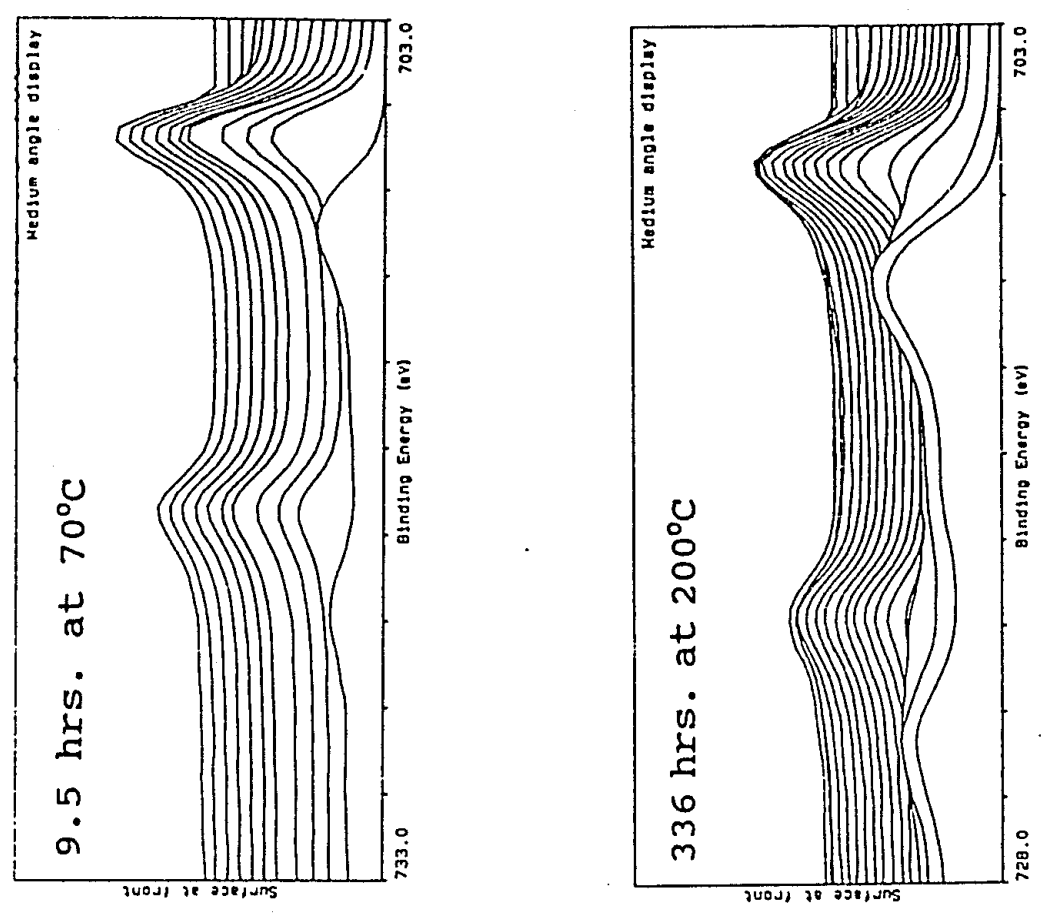

H્

㟧
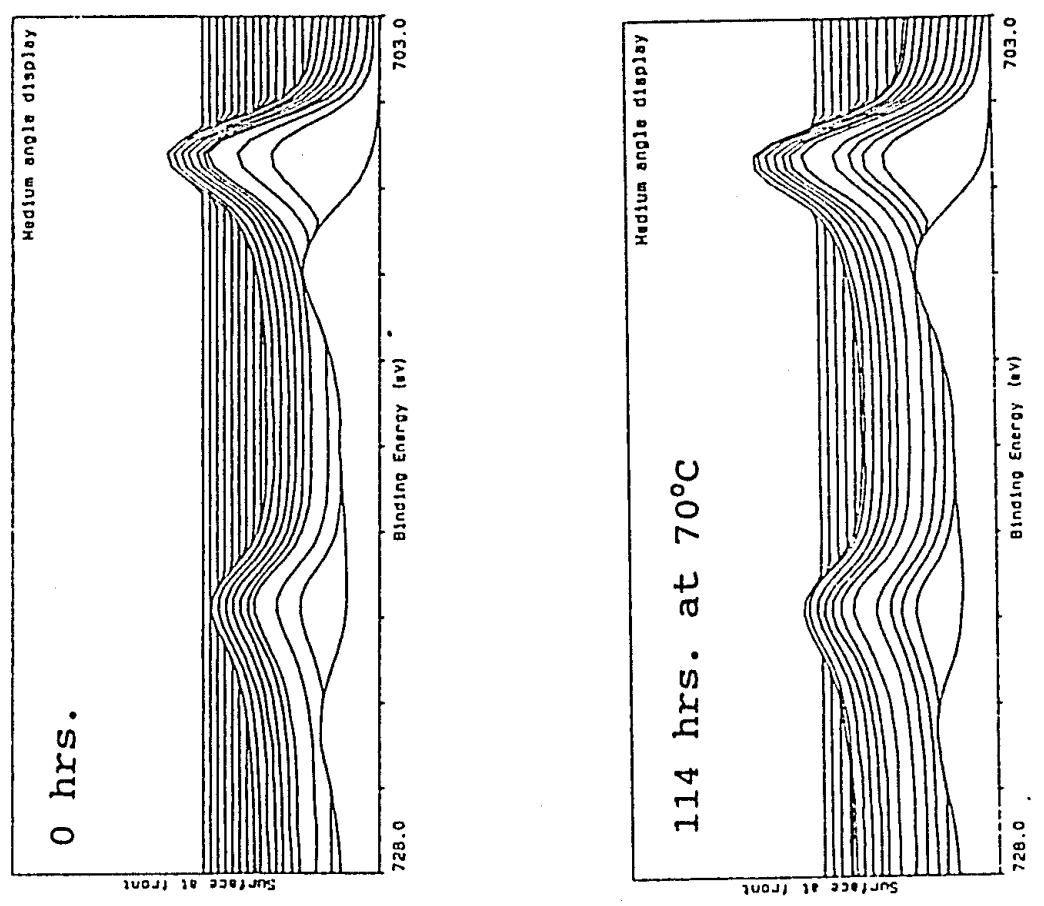

告

बू

ن

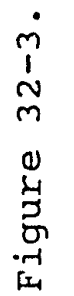



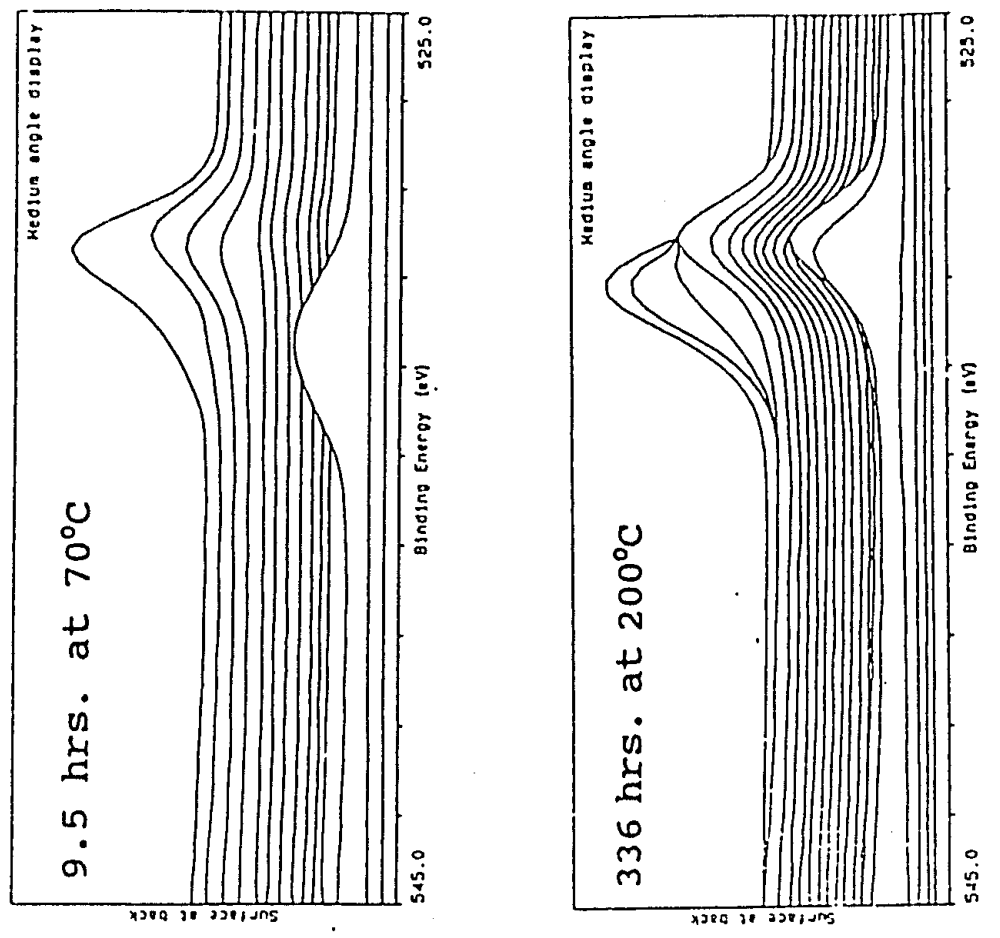

Oू
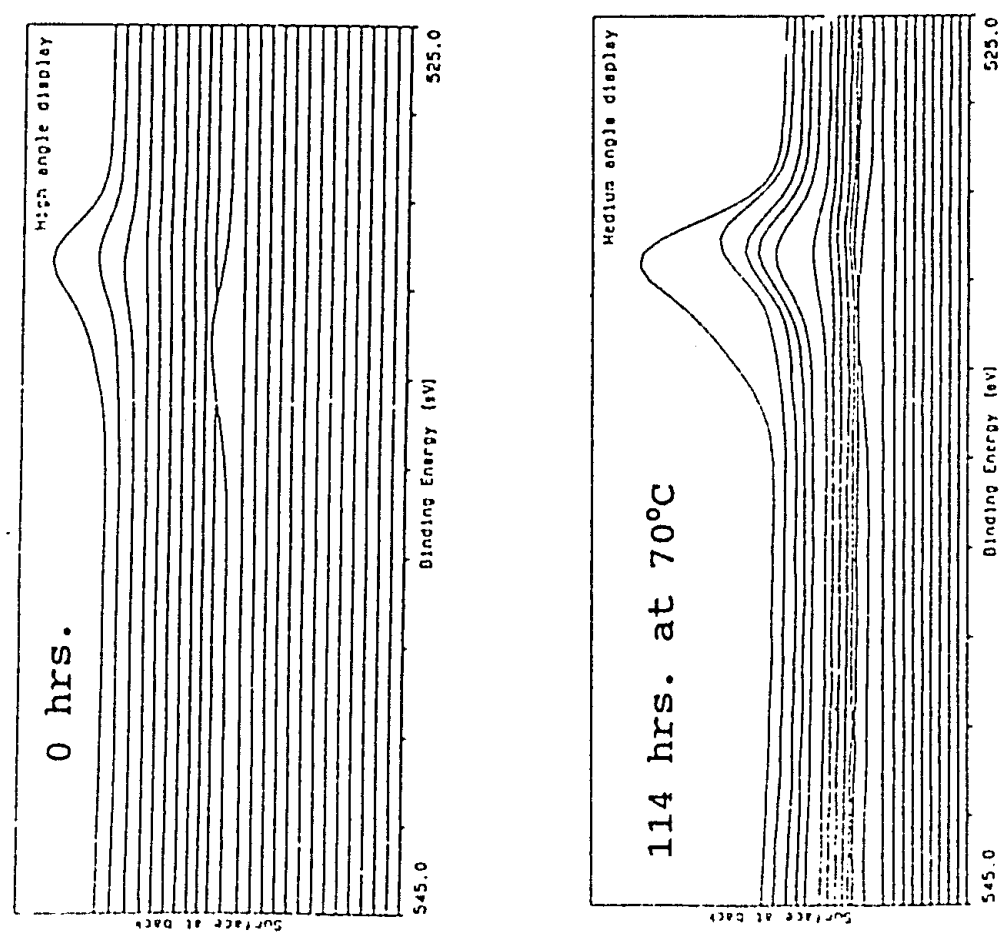

4

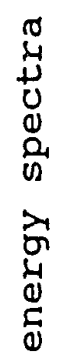

吕

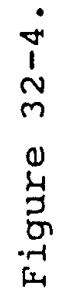


$\forall$
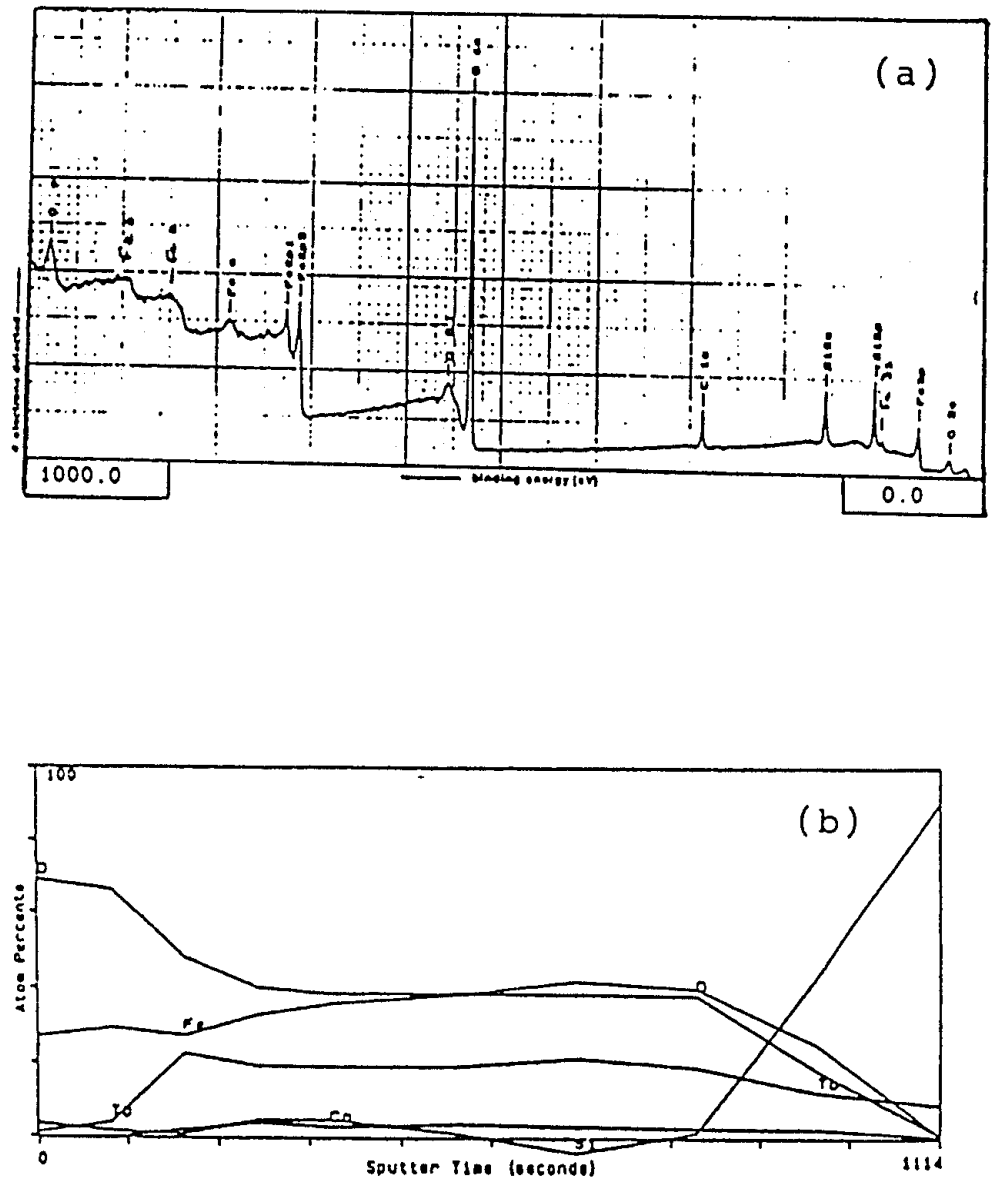

Figure 33-1. (a) Surface energy spectrum and (b) ESCA depth profiling; of TbFeco films annealed at $200^{\circ} \mathrm{C}$ for 336 hours. 

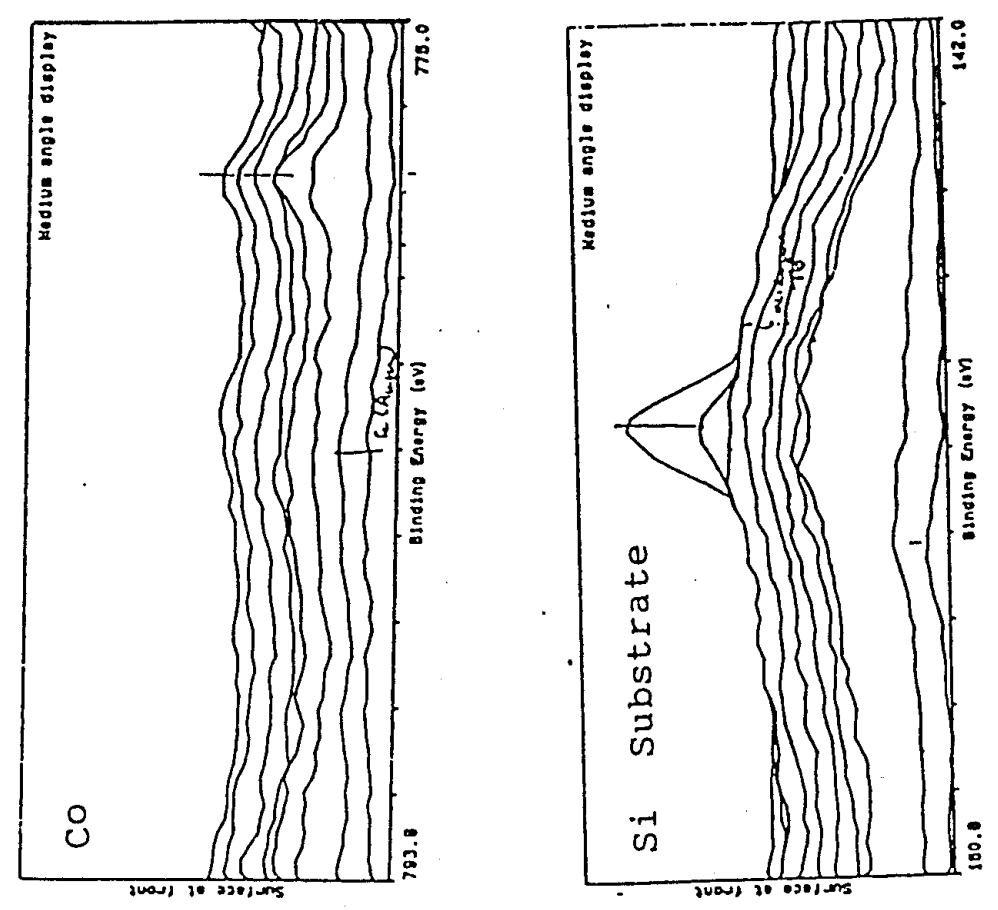

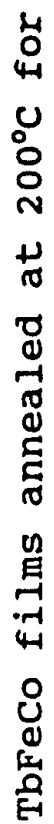
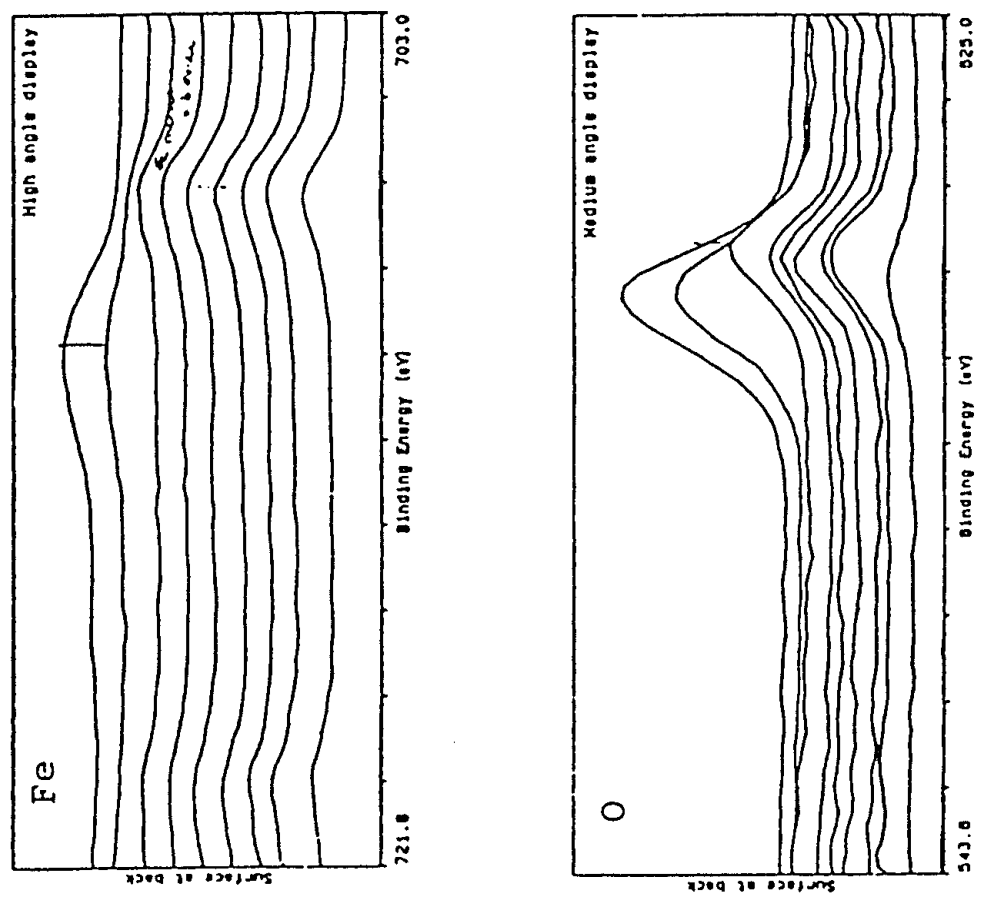

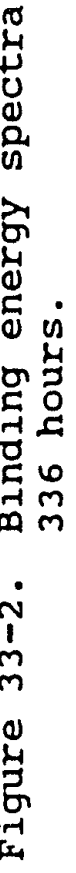


2 weeks at $200^{\circ} \mathrm{C}$. It is noteworthy that when annealed for 2 weeks at $200^{\circ} \mathrm{C}$, TbFe as well as TbFeco samples developed a top surface layer with Fe and oxygen only, with less than 18 $\mathrm{Tb}$ or Co at the surface. This is in agreement with the results of Van Dover et al. (45) that show the presence of a thin iron oxide layer at the surface. The thickness of the iron oxide layer is about $25 \AA$ for TbFeCo and 25-50 $\AA$ for TbFe samples. As in the study of TbFeco films, (53) the oxide depth for TbFe films was assumed to correspond to the point where the oxygen concentration in the film was $10 \%$.

Excluding the films treated for 336 hours at $200^{\circ} \mathrm{C}$, the surfaces of these films were richer in terbium as compared to the bulk film. In the as-deposited, air-exposed samples, the depth of $\mathrm{Fe}$ and Co oxidation is less than $25 \AA$ for TbFeco films and about $50 \AA$ for TbFe films. There also appears to be an increase in $\mathrm{Tb}$ and oxygen concentration at the filmsubstrate interface. The source of this oxygen might be the substrate surface, or it might have been trapped or absorbed during film deposition. This interface behaves very similarly to the film/air interface, except for the fact that the oxidized zone does not appear to grow with time, though more and more Tb appears to move towards the interface. This may be due to the limited availability of oxygen at the filmsubstrate interface. 


\subsection{Changes in Film structure with oxidation}

As stated earlier, the TbFe and TbFeco films were deposited on a $3 \mathrm{~mm}$ Cu grid for TEM analysis. In addition, samples on $\mathrm{Si}$ substrates were thinned down in a wedge shape to study in tine TEM. These samples of TbFe and TbFeCo films studied in the TEM exhibit oxidation on the thin areas of the sample. Figure 34 shows the diffraction patterns and bright field images at $100 \mathrm{~K}$ magnification for $\mathrm{TbFe}$ and TbFeCo non-annealed samples, with SiN protective overcoats for a thick area. Both of the films look amorphous in nature and dense in appearance. Imaging of the samples on the thin area shows part of the film to be oxidized (in case of TbFeCo), clearly showing the oxidized and non-oxidized areas with the oxidation front (see Figure 35-1). The TbFe films show larger and more numerous crystallites, as compared to the TbFeco films. The TbFe films also show sharper diffraction pattern indicating a higher degree of crystallization (fig. 35-2). In the case of $\sin$ overcoated films, the films deposited on $\mathrm{Cu}$ grids were also dense and compact in the asdeposited condition. The bare films of TbFe and TbFeco undergo oxidation. The TEM reveals the film structure similar to the one observed on thin areas of $\mathrm{siN}$ overcoated samples. 

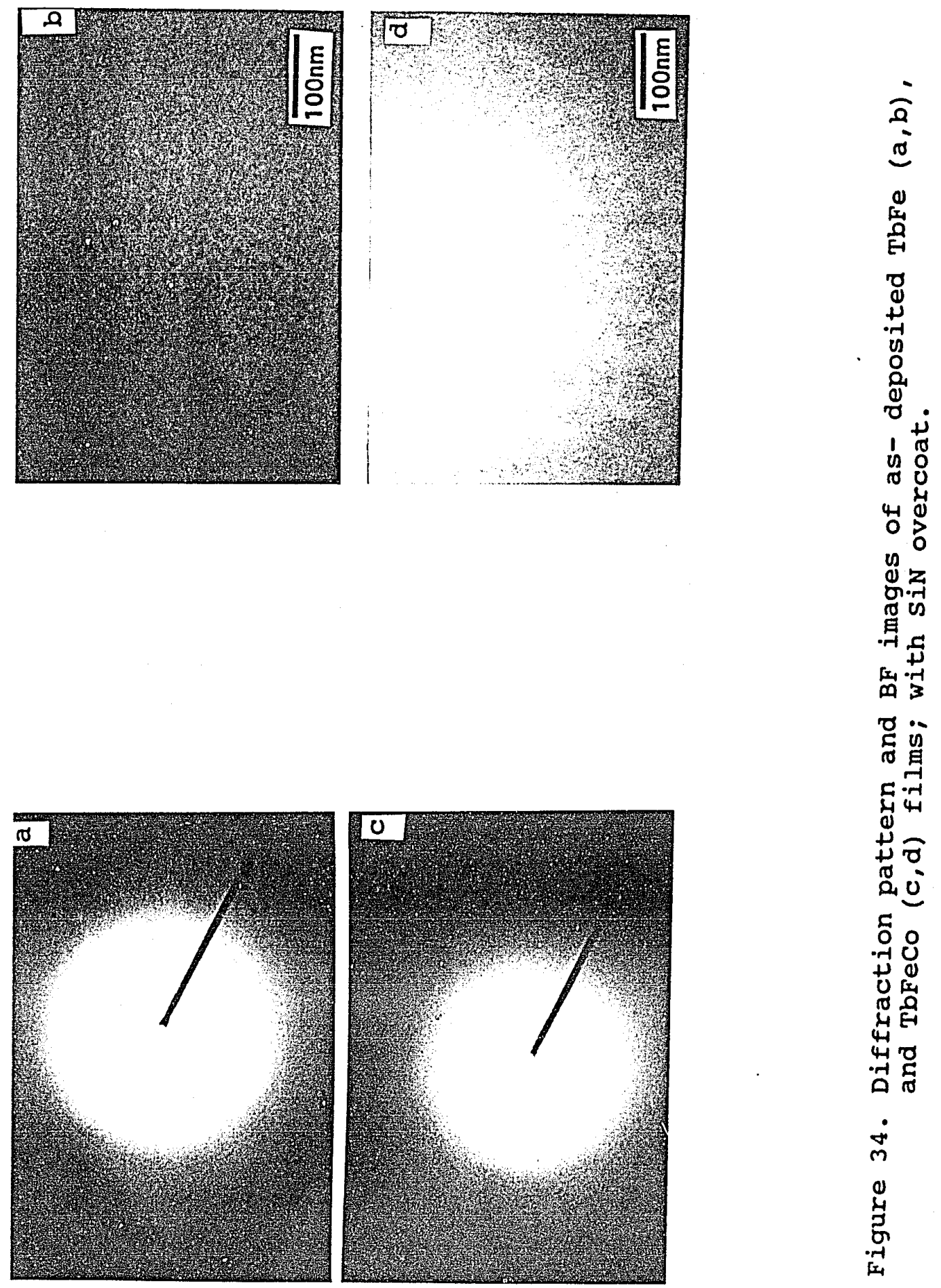

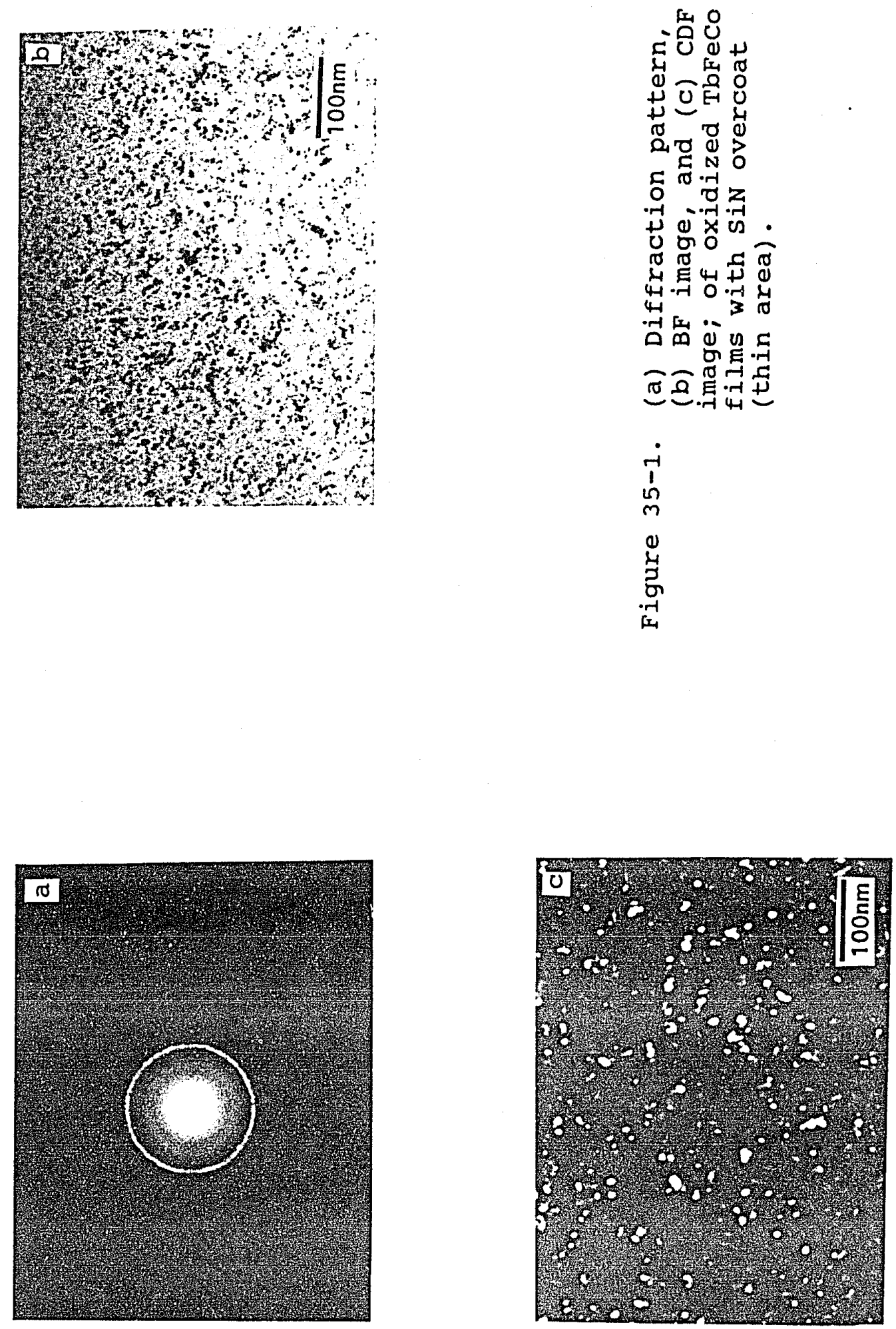


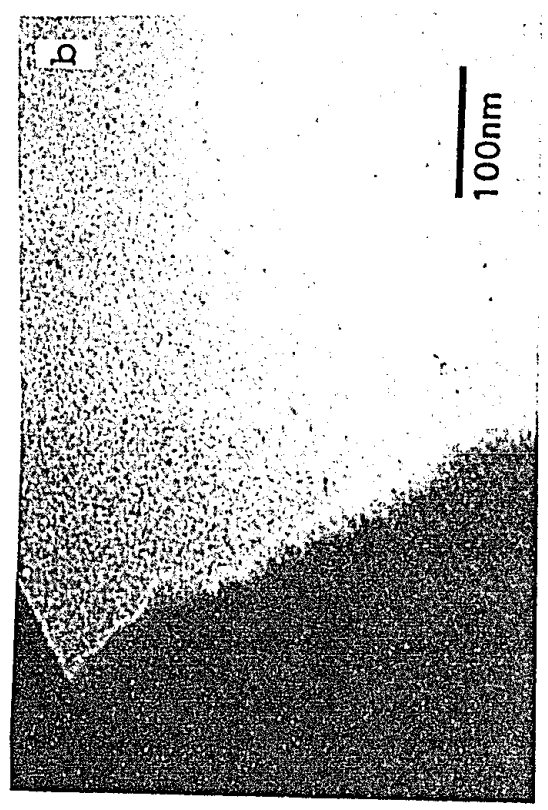

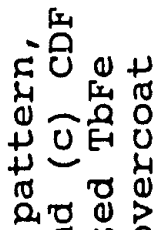

⿷匚

年

o 察

त

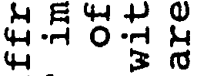

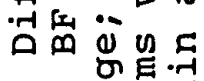

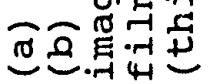

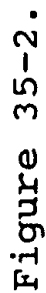
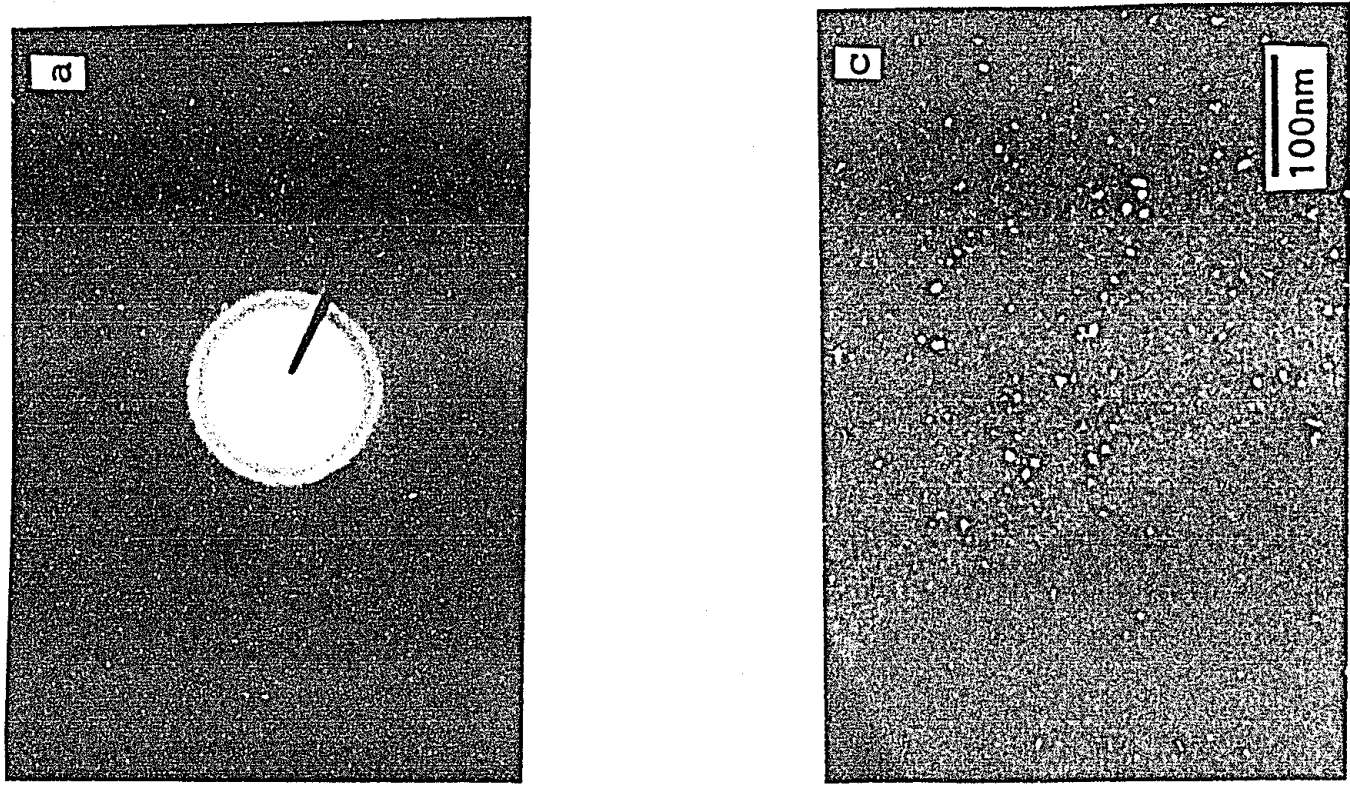


\subsection{Discussion of Results}

Annealing unprotected films of TbFe and TbFeco results in the preferential oxidation of $\mathrm{Tb}$ as compared to that of Co and Fe. During the oxidation process, a bare TbFe or TbFeCo film consist of three regions. The surface layer (about 25 A) consists of a mixture of oxides of terbium and transition metal components. Underneath this surface, Fe and Co exist in a metallic form in a matrix of $\mathrm{Tb}_{x} \mathrm{O}_{y}$, forming an oxide growth zone. With annealing of the samples at $70^{\circ} \mathrm{C}$, oxygen diffusion leads to the parabolic growth of the $\mathrm{Tb}_{\mathrm{x}} \mathrm{O}_{y}$ layer. This is shown in Figure 36, where the thickness of the 10 at: oxygen penetration is plotted with respect to time ${ }^{1 / 2}$. This region has increased concentration of $\mathrm{Tb}$, which arises due to the diffusion of $\mathrm{Tb}$ from the bulk to the surface of the film. The depth of the oxide growth zone increases at the expense of the unoxidized film. The Fe and co oxidation takes place at a much slower rate. Even for 400 hours of annealing at $70^{\circ} \mathrm{C}$, the $\mathrm{Fe}$ and co oxide depths are estimated to be only about $50 \AA$. The rest of the film is unoxidized. A schematic of the three zones is shown in Figure 37.

It is clear that the properties of these films are severely modified by the compositional changes in the film. The existence of $\mathrm{Fe}$ and $\mathrm{Co}$ in the metallic form inside the $\mathrm{Tb}_{x} \mathrm{O}_{y}$ matrix modifies the magnetic and magneto-optical behavior of these films. The effect of this layer on the 


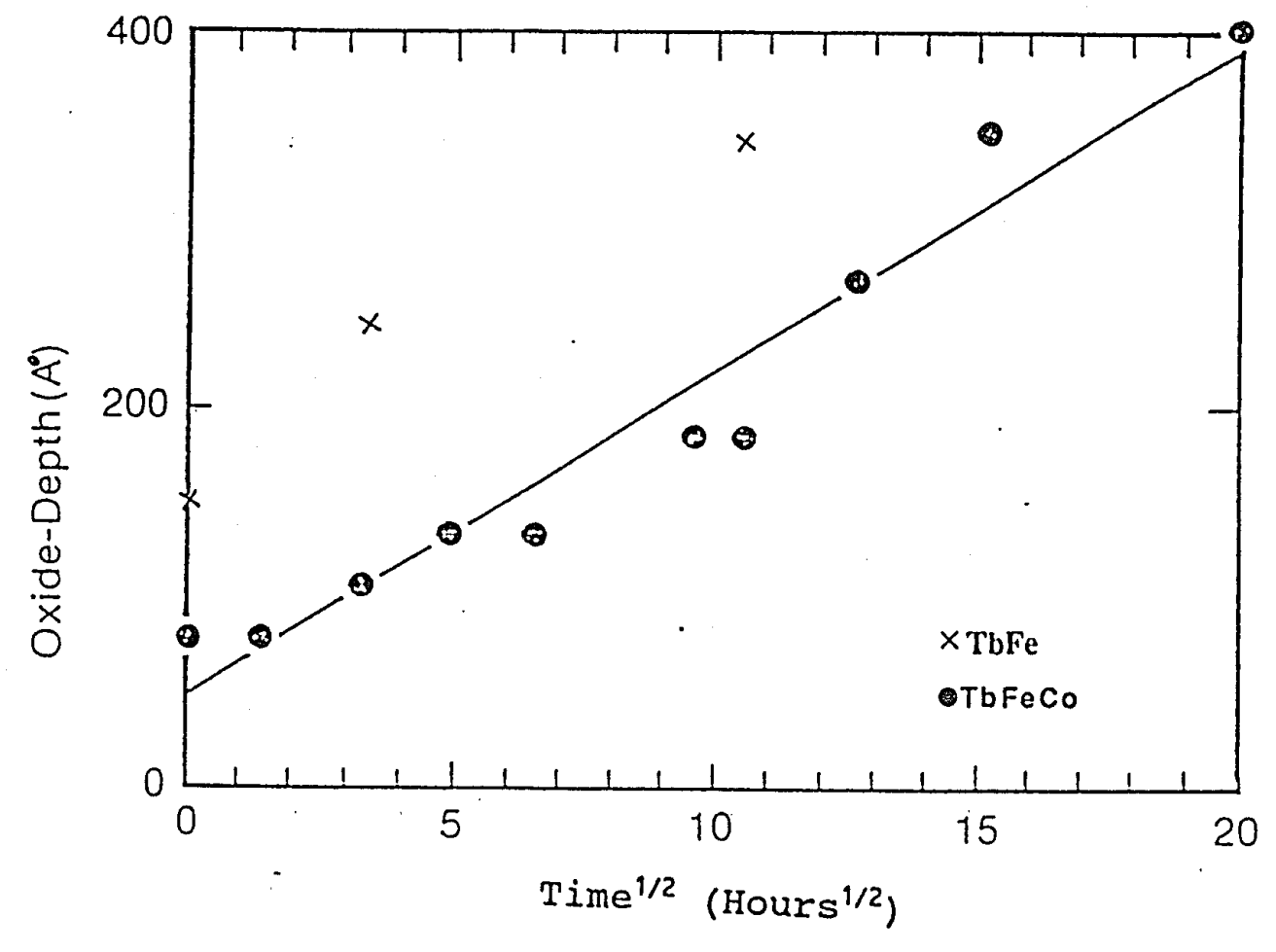

Figure 36. Oxidation depth as a function of $t i m e^{1 / 2}$, for TbFe and TbFeCo films annealed at $70^{\circ} \mathrm{C}$. 


\begin{tabular}{c}
\hline Oxides of Tb \& Transition Metals \\
\hline Fe and Co dispersed in TbxOy \\
\hline
\end{tabular}

Unoxidized Layer

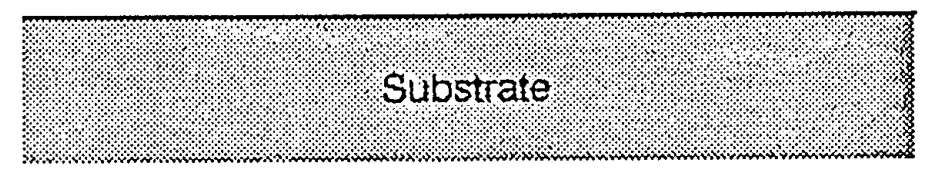

Figure 37. Schematic of compositional changes with oxidation for TbFe and TbFeCo films. 
growth of an in-plane component of magnetization, the decrease in coercivity upon oxidation, and the non-saturating behavior of the Kerr loops will be explained later. On the other hand, terbium oxide is relatively transparent and does not exhibit magneto-optical behavior. (53) Thus, the surface layer of terbium oxide is not expected to contribute to changes in the magnetic behavior of these films with annealing. It may, however, modify the optical behavior of the signal due to its interaction with a light beam. (53)

As can be seen from Figure 7 , the coercivity of the transition metal-rich films is expected to decrease with a decrease in the $\mathrm{Tb}$ concentration. This explains the observed decrease in coercivity of annealed TbFe and TbFeCo samples. The following experiment was conducted to estimate the effect of the metallic Fe and Co dispersed inside the terbium oxide matrix. Fe overlayers of $28 \AA, 94 \AA$, and $151 \AA$ thicknesses were deposited on $100 \mathrm{~nm}$ thick TbFe films. In order to prevent any oxidation effect, these films were protected with an overlayer and an underlayer of $400 \AA$ of $\mathrm{SiN}$. The Kerr rotation and VSM hysteresis loops for these films are shown in Figure 38. These figures show that the increase in the Fe overlayer thickness causes the Kerr loops to be increasingly slanted. This is because Fe has its hard axis normal to the film plane. As discussed earlier, the slopes of the Kerr loops for TbFe and TbFeCo films with oxidation exhibits a similar behavior as shown in Figures 20 and 21 . This is an 


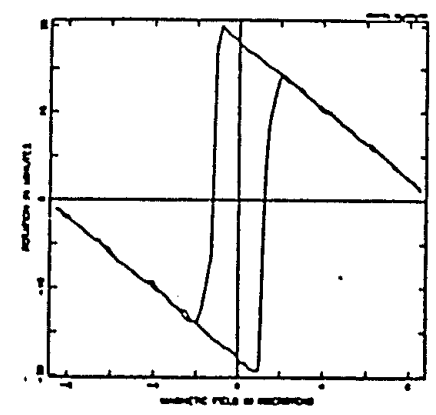

(a)
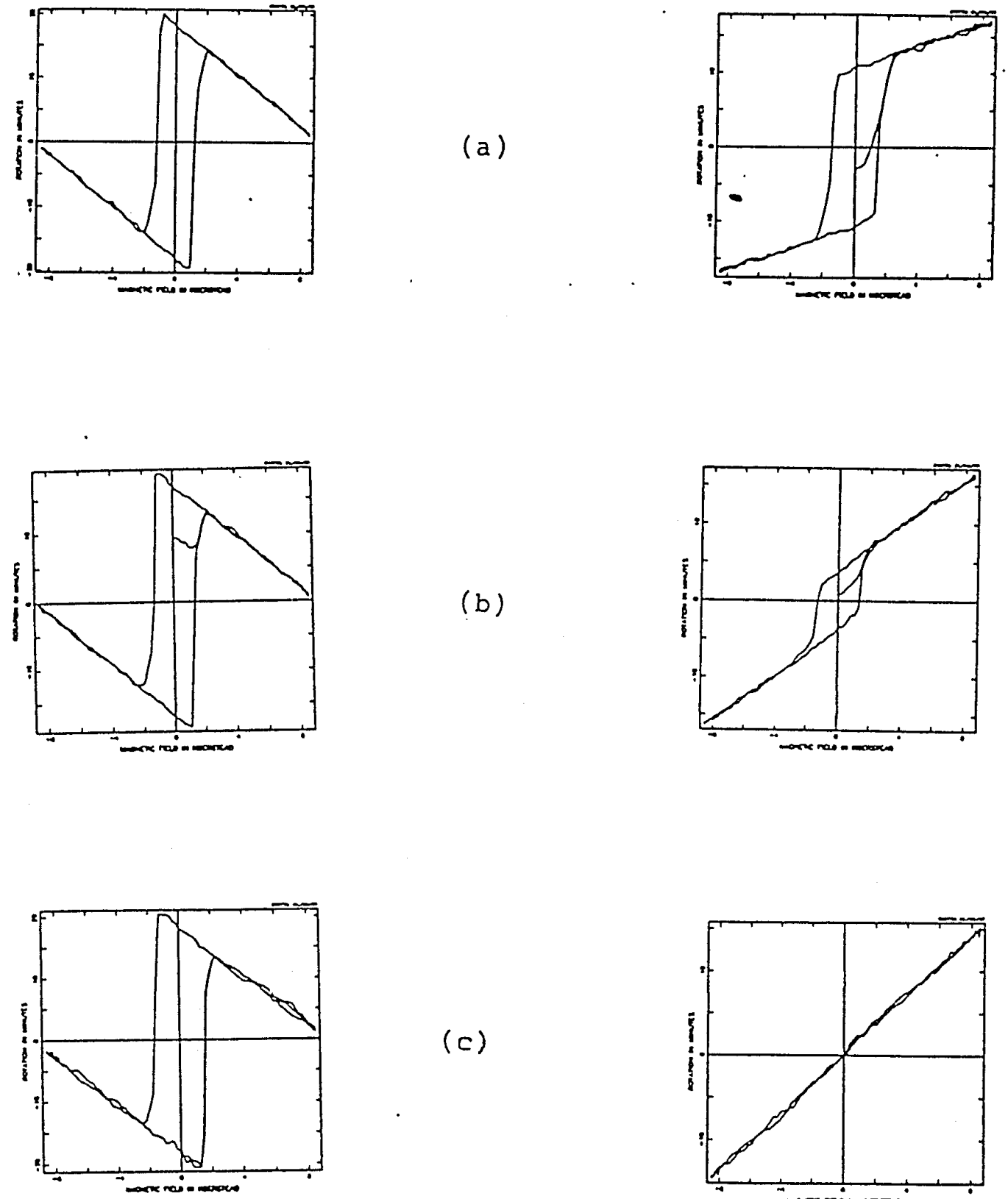

(b)

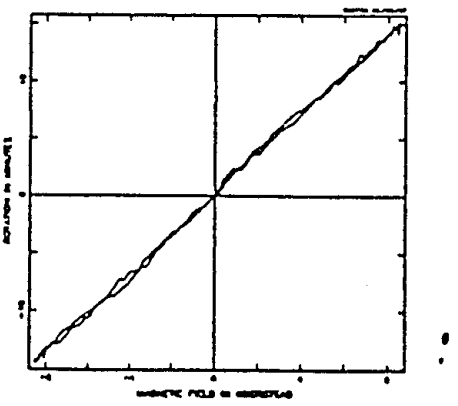

Figure 38-1. Kerr loops for (40 nm SiN/ xFe/ $100 \mathrm{~nm}$ FeTb/ $40 \mathrm{~nm}$ SiN/ substrate) films for $x=$; (a) $2.8 \mathrm{~nm}$, (b) $9.4 \mathrm{~nm}$, (c) $15.1 \mathrm{~nm}$. 


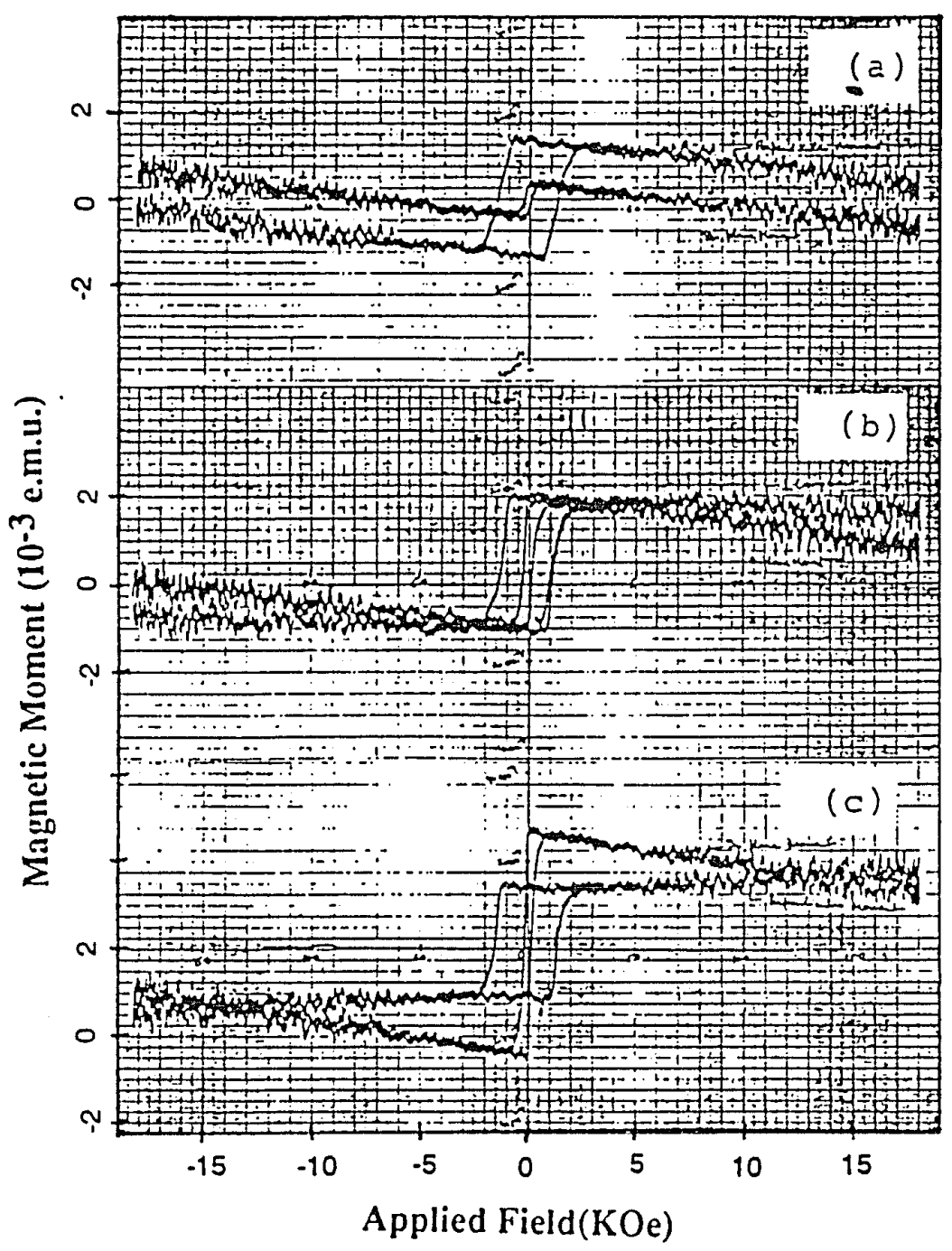

Figure 38-2. VSM hysteresis loops for (40 nm SiN/ $\mathrm{xFe} /$

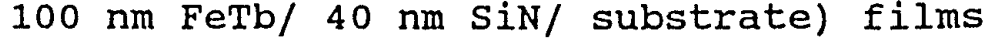
for $x=$; (a) $2.8 \mathrm{~nm}$, (b) $9.4 \mathrm{~nm}$, (c) 15.1 nm. 
additional evidence of the presence of metallic Fe and co over the RE-TM film. From the Fe overlayer samples, it is estimated that the initial non-annealed, air-exposed samples have the equivalent of about 25 of Fe in the oxidized zone. The samples annealed for 114 hours contain an equivalent Fe layer thickness of about $80 \AA$. The decrease in the fiim incident Kerr rotation with the increase in Fe overlayer thickness is due to the absorption of the laser beam by Fe. As expected, the substrate incident Kerr rotation of the Fe overlayer samples does not exhibit any change. Terbium oxide is relatively transparent. Therefore, the decrease in Kerr rotation angle for the annealed TbFe and TbFeCo films cannot be explained by the presence of metallic Fe and co dispersed inside terbium oxide matrix.

The TbFe and TbFeco films under investigation, being rich in $\mathrm{Fe}$, have a positive loop sense as shown for films with 19\% Tb in the upper half of Figure 6 . Upon oxidation, there is a further decrease in the concentration of $\mathrm{Tb}$ in the film. Thus, the decrease in Kerr rotation and reversal in the sense of Kerr loop cannot be explained on the basis of a change in the Tb/Fe ratio during oxidation. These observations are explained as below. In the oxidized films, the elliptically polarized reflected light originating from the unoxidized RETM film undergoes further optical changes as it travels through the oxidized region. This can be modeled by optical theory. (58) 
Figure 39 shows computed Kerr rotation and ellipticity values for a two-layer structure consisting of an absorbing but nonmagneto-optic layer with refractive index $N=N^{+}=N^{-}=$ 3.0-i2.0 over a magneto-optic layer with a refractive index $\mathrm{N}^{+}=3.68-\mathrm{i} 3.852$ and $\mathrm{N}^{-}=3.59-\mathrm{i3} .794$, as reported for $\mathrm{TbFe} .{ }^{(59)}$ The thickness of the nonmagnetic overlayer is varied in the calculation, and its optical constants were chosen to be representative of the absorptive oxidized surface layer. The calculation predicts the progressive reduction in Kerr angle and also the reversal in Kerr loop sense as observed in the surface oxidation experiment. In particular, the Kerr ellipticity is initially predicted to increase, before the absorption in the surface layer overwhelms the Mo response (at a layer thickness of about $140 \mathrm{~nm}$ ), when essentially none of the incident beam can penetrate to the underlying mo layer.

Experimentally, this predicted behavior is confirmed by remeasuring the rotation from an oxidized sample with the insertion of a phase-compensation plate into the beam path of the light reflected from the oxidized surface. The phase plate was adjusted to bring the components of polarization of the reflected beam into phase, so that the effective Kerr angle now includes the ellipticity signal, i.e., $\theta_{k}{ }^{p p}=\left(\theta_{k}{ }^{2}+\epsilon_{k}{ }^{2}\right)^{1 / 2}$, where $\theta_{k}$ and $\epsilon_{k}$ are the kerr rotation and ellipticity, respectively, in the absence of the phase compensation. ${ }^{(60)}$ The Kerr rotation measurement in the 


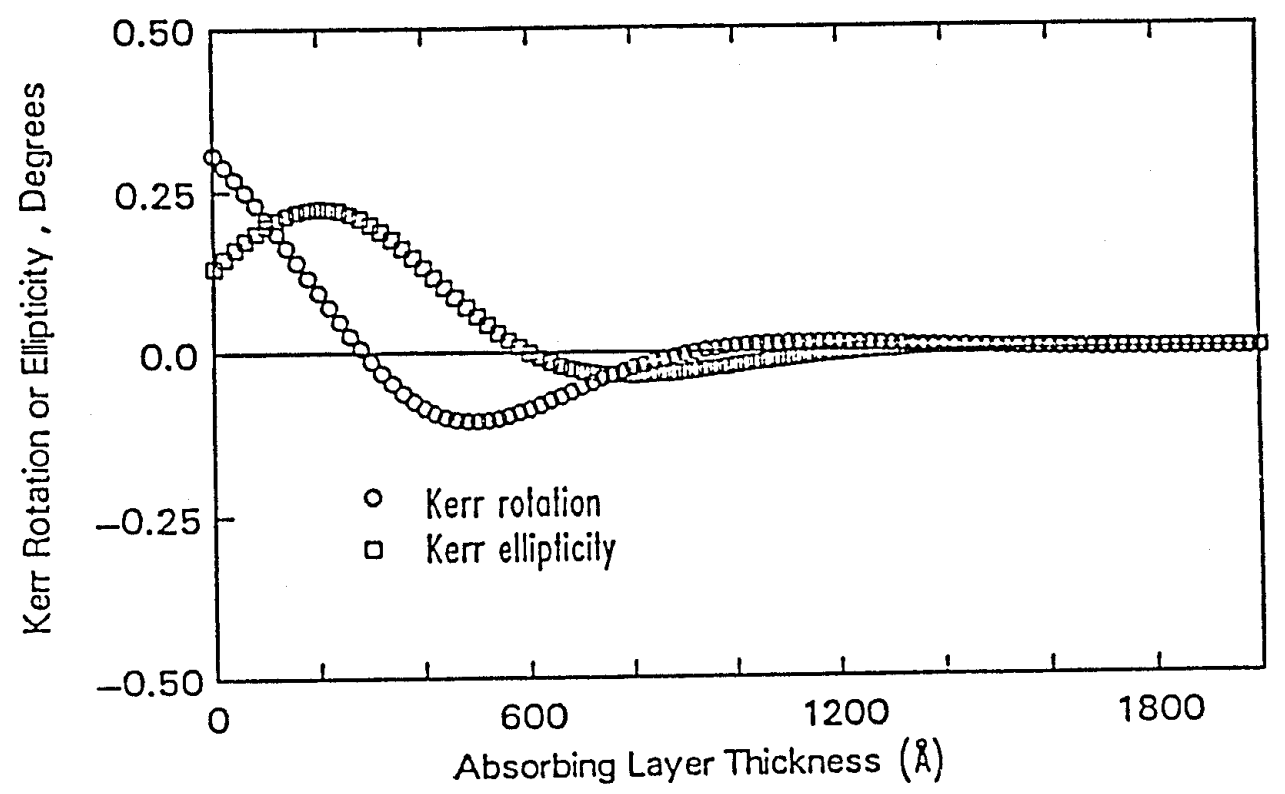

Figure 39. Computed Kerr rotation and ellipticity originating from a two-layer structure consisting of an Mo layer overcoated with an absorbing but nonmagneto-optically active layer. (59) 
presence of the phase compensation resulted in a considerable increase in the hysteresis loop amplitude, as shown in Figure 40. This confirms the presence of significant ellipticity in the MO response of this oxidized sample. The change in the reflectivity of these films was also measured as a function of annealing time. As can be seen in Figure 41, the reflectivity decreases to about $70 \%$ of the initial value after 114 hours of oxidation time for TbFe films and after 398 hours of oxidation time for TbFeco films. Even though most of the loss in Kerr rotation of these oxidized films can be optically recovered, the decrease in reflectivity of these films dramatically reduces the light sensed by the polarization detectors in an optical device. This reduction in the reflected light from oxidized samples is unacceptable for recording applications.

Oxidation of $\mathrm{TbFe}$ and TbFeco samples, at $200^{\circ} \mathrm{C}$ for 2 weeks, shows that the growth of the oxide zone in the film continues until oxygen has penetrated through the whole film. After this time, it appears that Fe starts to migrate to the surface as is indicated by the ESCA depth profile of these samples. As can be seen from the depth profile of these samples (Figures $29 \& 30$ ), the surface layer at this time is rich in $\mathrm{Fe}$ but depleted in $\mathrm{Tb}$. The comparison of the oxidation behavior of the two films indicate that the oxidation mechanism is similar in the two cases. However, oxidation proceeds at a much slower rate in TbFeco films than 


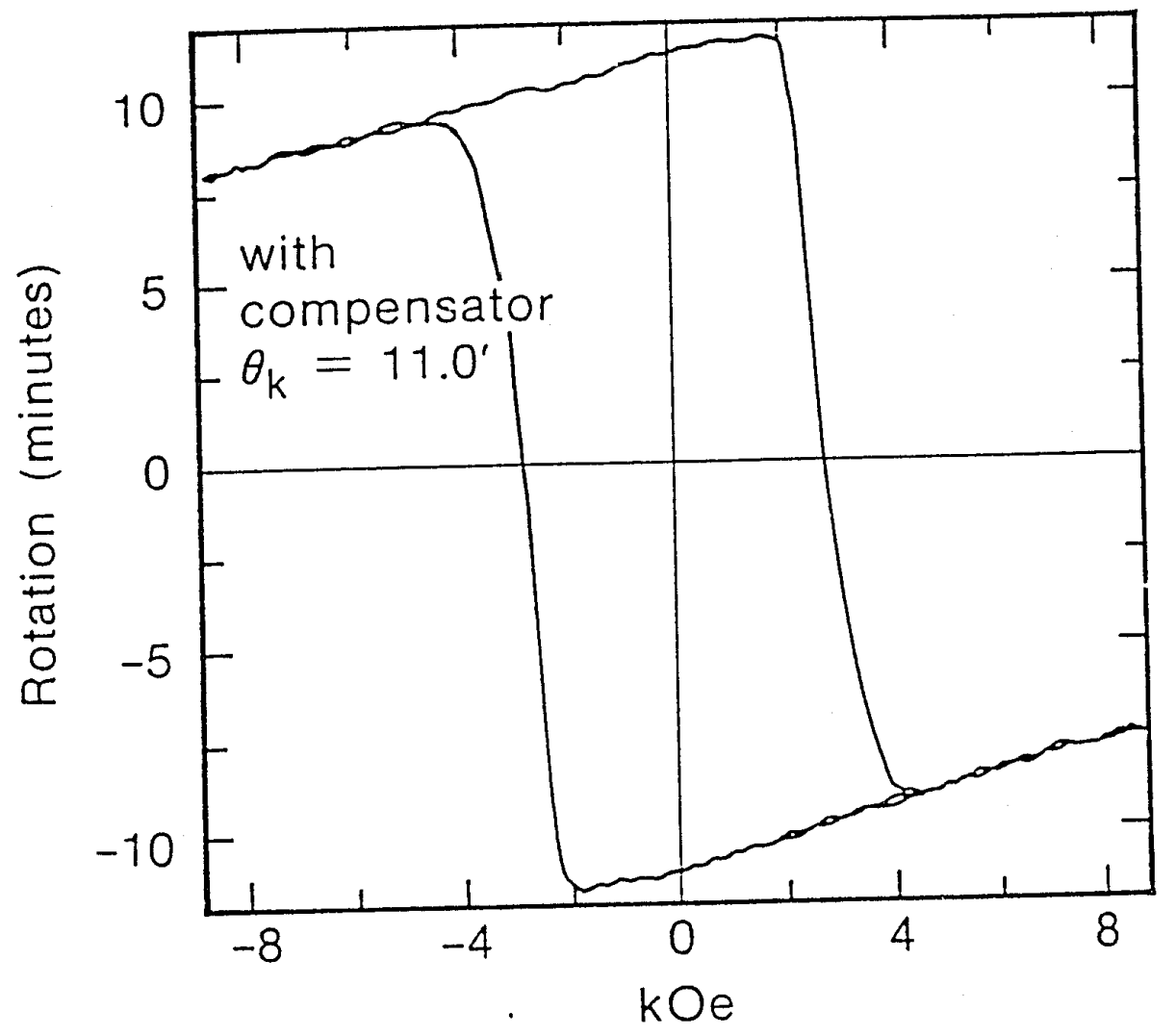

Figure 40. Kerr loop of TbFeCo a film, annealed for 72 hours at $70^{\circ} \mathrm{C}$, with compensator placed in optical setup. 


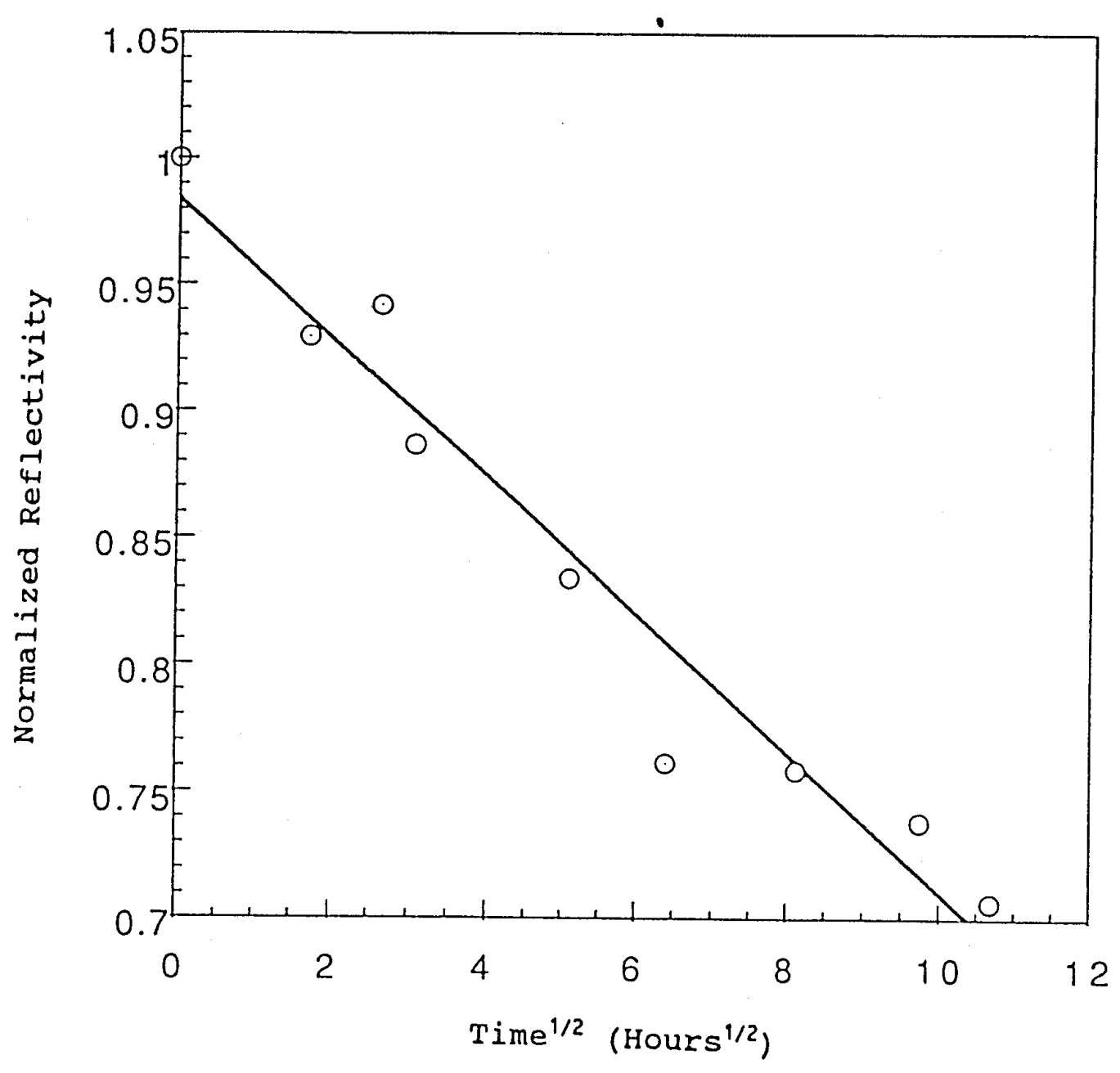

Figure 41. (a) Normalized reflectivity of TbFe films annealed at $70^{\circ} \mathrm{C}$ as a function of time 


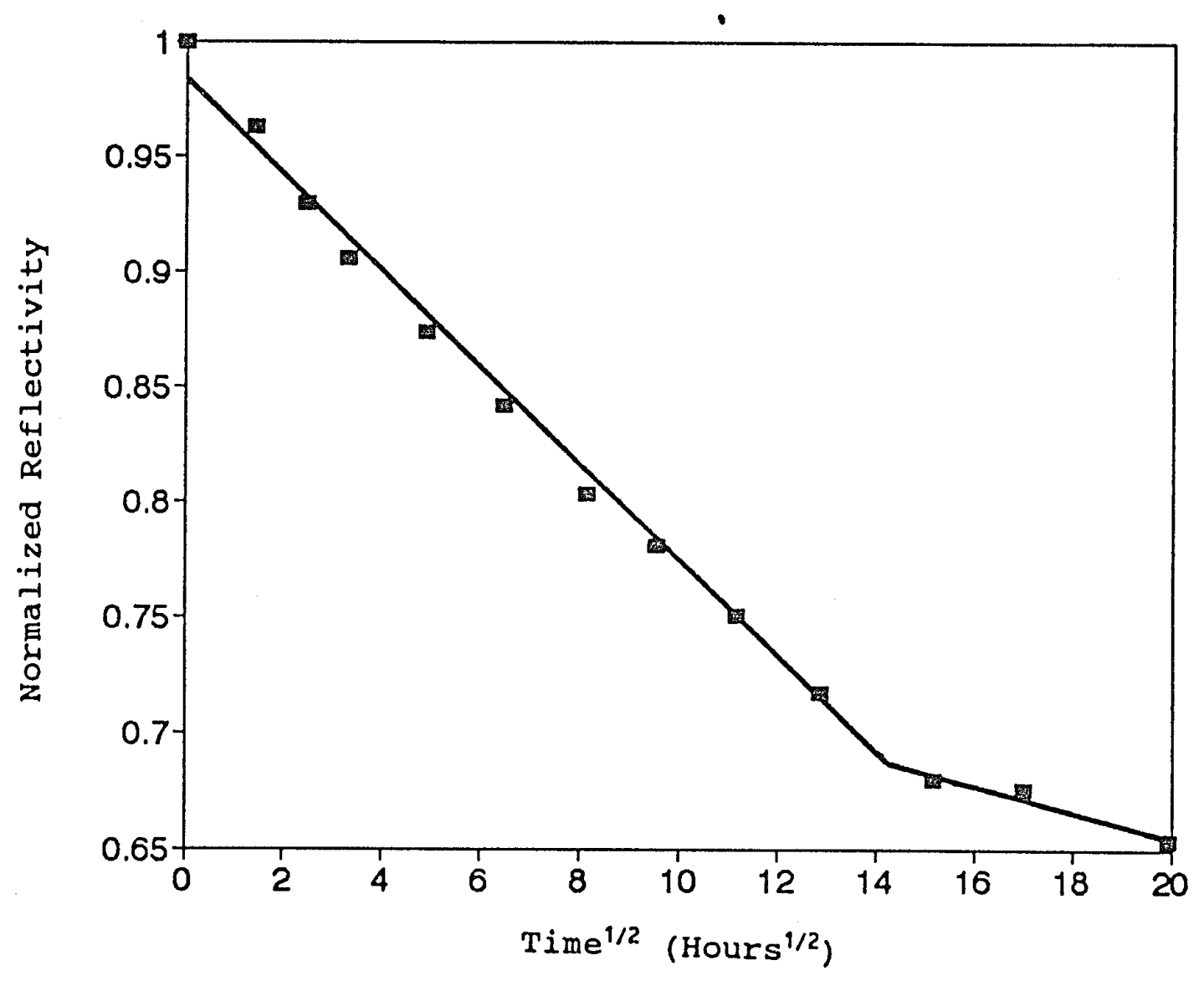

Figure 41. (b) Ncrmalized reflectivity of TbFeco films annealed at $70^{\circ} \mathrm{C}$ as a function of $t i m e^{i / 2}$. 
in the TbFe films. The degradation in film properties for TbFe films in about 114 hours is similar to the changes in TbFeCo films in 400 hours, i.e., the oxidation rate in TbFe films is at least 3 times faster than the oxidation rate of TbFeCo films. 
Chapter 5

\section{CONCLUSIONS}

The as-deposited TbFe and TbFeCo films prepared with DC magnetron sputtering are dense and amorphous. This is indicated by TEM images and diffused diffraction patterns. The oxidation of these bare films results in time and temperature dependent compositional changes. The rare earth component of these films is very reactive. Upon annealing the samples at $70^{\circ} \mathrm{C}$, the terbium oxide grows linearly with $t_{i m e^{1 / 2}}$. The parabolic growth rate of these films is indicative of the diffusion controlled oxidation process. The rates of oxidation of $\mathrm{Tb}, \mathrm{Fe}$, and Co are different from each other, being the highest for $\mathrm{Tb}$.

This difference in the oxide growth rate of $\mathrm{Tb}$ and the transition metal components results in the development of a complex structure with annealing. The surface layer consists of mixed oxides of $\mathrm{Tb}, \mathrm{Fe}$, and Co. This oxidized surface layer modifies the optical properties, such as ellipticity and reflectivity, of the magneto-optical film. Underneath this layer, metallic $F e$ and Co (for TbFeco films) are dispersed in the matrix of terbium oxide. This mixed layer of terbium oxide and the transition metal components grows with oxidation. As a result, the magnetic and Kerr characteristics of these films are continuously modified 
during oxidation. These changes in the film properties are indicated by the non-saturating Kerr loops and growth in the parallel component of magnetic moment as measured by the VSM. The rate of degradation in the magneto-optical properties on annealing is much higher for TbFe films compared to that of TbFeco films. The oxidation rate of the TbFe films was found to be more than three times higher than that of the TbFeco films. 
Chapter 6

\section{RECOMMENDATIONS FOR FUTURE WORK}

A considerable amount of work has been done to understand the degradation mechanism of magneto-optical thin films. Below are some of the areas that may be further studied.

(a) The mechanism of improvement in the environmental stability of these films with the addition of alloying elements.

(b) Development of protective layers that will improve the corrosion resistance and enhance the signal. These protective films should not cause high stresses in the films.

(c) New materials and techniques, such as multilayer structures and Pt containing films, to improve environmental stability and magneto-optical properties.

The development work in these areas will further help in improving the environmental stability of magneto-optical recording media. 
Chapter 7

REFERENCES

1. G. A. N. Connell, "Problems and opportunities in Erasable optical Recording", AIP Conference Proceeding No. 138: Frontiers in Electronic Materials Processing, American Institute of Physics, (1987), p. 29 .

2. M. C. Gupta and F. Strome, "Erasable Laser Recording in an Organic Dye-binder Optical Disk Medium", I. Appl. Phys., Vol. 60, (1986), p. 2932.

3. A. B. Merchant, "Optical Recording", Addison Wisley Publishing Company, (1990), p. 67.

4. F. O. Sequeda and H. P. D. Shieh, "Optical Information storage Technology", IBM Internal Research Report, (March 1990), p. 28.

5. A. E. Bell, "Handbook of Laser Science and Technology, Vol. V, Optical Materials",CRC Press Inc., $(1987)$, p. 65 .

6. J. Isailovic, "Videodisc and optical Memory Systems", Prentice Hall, (1985), p. 4 .

7. P. Argyres, "Theory of the Faraday and Kerr Effects in Ferromagnetics", Phys. Rev., Vol. 97, (1955), p. 334 .

8. P. S. Pershan, "Magneto-optical Effects", J. Appl. Phys., Vol. 38, (1967), p. 1482 .

9. M. J. Freiser, "A Survey of Magneto-optical Effects", IEEE Trans. Magn., Vol. MAG-4, (1968), p. 152 .

10. H. J. Williams, R. C. Sherwood, F. G. Foster, and E. M. Kelly, "Magnetic Writing on Thin Films of MnBi", J. Appl. Phys., Vol. 28, (1957), p. 1181.

11. L. Mayer, "curie Point Writing on Magnetic Films", J. Appl. Phys., Vol. 29, (1958), p. 1003 .

12. L. Burns and E. Keizer, "Magnetic Recording System", U.S. Patent No. 2915594, (1959). 
13. D. Chen, J. Ready, and G. E. Bernal, "MnBi Thin Films; Physical Properties and Memory Applications", J. Appl. Phys., Vol. 39, (1968), p. 3916.

14. K. Lee, "Magnetic Thin Films for Optical storage", J. Vac. Sci. Tech., Vol. 10, (1973), p. 631.

15. P. Chaudhari, J. J. Cuomo, and R. J. Gambino, "Amorphous Metallic Films for Magneto-optic Applications", Appl. Phys. Lett., Vol. 22, (1973), p. 337.

16. Y. Togami, "Magneto-optic Disk Storage", IEEE Trans. Magn., Vol. MAG-18, (1982), p. 1233.

17. M. H. Kryder, "Magneto-optic Recording Technology", J. Appl. Phys., Vol. 57, (1985), p. 3913.

18. W. H. Meiklejohn, "Magnetooptics: A Thermomagnetic Recording Technology", Proc. of the IEEE, Vol. 74, $(1986)$, p. 1570 .

19. N. Sato, "Crystallography and Magnetism of Co-Pd and Co-pt Films with an Artificially Layered structure", J. Appl. Phy., Vol. 11, (1988), p. 6424 .

20. H. Do, "Properties of Evaporated Co/Pt Multilayer Thin Films for optical Recording", M.S. Thesis, San Jose State University, (May 1990).

21. M. Gomi, K. Sato, and M. Abe, "Improvements in Optical and Magnetic Properties of Bi-substituted Garnet Sputtered Films for Magneto-optical Recording", J. Appl. Phys., Vol. 63, (1988), p. 3642 .

22. P. G. Van Engen, K. H. J. Buschow, R. Jongebreur, and M. Erman, "PtMnSb, a Material with Very High Magneto-optical Kerr Effect", Appl. Phys. Lett., Vol. 42, (1983), p. 202 .

23 C. Zenner, "Interaction between the d Shells in the Transition Metals", Phys. Rev., Vol. 81, (1951), p. 440 .

24. M. Mansuripur and G. A. N. Connell, "Signal and Noise in Magneto-optic Readout", J. Appl. Phys., Vol. 53, (1982), p. 4485 .

25. H. P. D. Shieh, "Magneto-optic Recording MediaFabrication, Characterization, and optimization", Ph.D. Thesis, Carnegie Mellon University, (1986). 
26. C. J. Lin, "Reversible Optical storage", IBM Corporation, Internal Report, (1988), p. 2 .

27. F. E. Luborsky, "Kinetics for Changes in Anisotxopy, Coercivity and Argon Content of Transition MetalRare Earth Films", J. Appl. Phys., Vol. 57, (1985), p. 3592 .

28. C. D. Wright, P. J. Grundy, and E. T. M. Lacey, "Stability Phenomena in Amorphous Rare Earth-Transition Metal Films", IEEE Trans. Magn,, Vol. MAG-23, (1987), p. 162 .

29. S. Nakahara, M. Hong, R. B. van Dover, E. M. Gyorgy, and D. D. Bacon, "Microstructures of Thin Sputtered Amorphous $\mathrm{Tb}_{0.26} \mathrm{Fe}_{0.74}$ and Polycrystalline Fe Films", J. Vac. Sci. Tech., Vol. 4, (1986), p. 543.

30. S. Shin, "Dependence of Microstructure and Magnetooptical Properties on Deposition Angle in Evaporated TbFe and TbFeCo Films", J. Appl. Phys., Vol. 61, $(1987)$, p. 3592 .

31. G. A. N. Connell, R. Allen, and M. Mansuripur, "Magneto-optical Properties of Amorphous Terbiumiron Alloys", J.Appl. Phys., Vol. 53, (1982), p. 7759.

32. Mimura, Y. N. Imamura, T. Kobayashi, A. Okada, and Y. Kushiro, "Magnetic Properties of Amorphous Alloy Films of Fe with Gd, Tb, DY, Ho, or Er", J. Appl. Phys., Vol. 49, (1978), p. 1208.

33. D. S. Bloomberg and G. A. N. Connell, "Magnetooptical Recording", Magnetic Recording, Vol. II, McGraw Hill Book Co., (1988), p. 394 .

34. H. Tsujimoto, M. Shouji, A. Saito, S. Matsushita, and Y. Sakurai, "Magnetic Properties and Magnetic Kerr Rotation of Amorphous TbFeCo and TbFeCr Films", J. of Magnetism and Magnetic Materials, Vol. 35, (1983), p. 199.

35. R. B. van Dover, M. Hong, E. M. Gyorgy, J. F. Dillon, and S. D. Albiston, "Intrinsic Anisotropy of TbFe Films Prepared by Magnetron Co-sputtering", J. Appl. Phys., Vol. 57, (1985), p. 3897 .

36. R. Hasegawa, R. J. Gambino, J. J. Cuomo, and J. F. Ziegler, "Magnetization of Amorphous Gd-CO-Ni Films", J.Appl. Phys., Vol. 45, (1974), p. 4036. 
37. G. Bate, "Material challenges in Metallic, Reversible, optical Recording Media", IEEE Trans. Magn., Vol. MAG-23, (1987), p. 156.

38. X. Yan, M. Hirscher, T. Egami, and E. Marinero, "Direct Observation of Anelastic Bond-orientational Anisotropy in Amorphous $\mathrm{Tb}_{26} \mathrm{Fe}_{62} \mathrm{Co}_{12}$ Thin Films by Xray Diffraction", phy. Rev. B., Vol. 43, (1991), p. 9300.

39. M. Hirscher, T. Egami, and E. Marinero, "Atomistic study of Magneto-optical Amorphous Thin Films using Synchrotron Radiation", J. Appl. Phys., Vol. 67, (1990), p. 4932.

40. M. H. Kryder, H. P. Shieh, And D. K. Hairston, "Control of Parameters in Rare Earth-Transition Metal Alloys for Magneto-optic Recording Media", IEEE Trans. Magn., Vol. MAG-23, (1987), p. 165.

41. D. Stone, "3.5-Inch Rewritable Optical Drives", PC Magazine, Vol. 11, (1992), p. 235.

42. M. M. Yang and T. M. Reith, "The Degradation of TbFeco Magneto-optic Media due to Reaction with Oxide Containing Dielectric Materials and substrates", J.Appl. Phys., Vol. 71, (1992), p. 3945.

43. R. Allen, and G. A. N. Connell, "Magneto-optic Properties of Amorphous Terbium-iron", I. Appl. Phys., Vol. 53, (1982), p. 2353.

44. P. Bernstein and C. Gueugnon, "Aging Phenomena in TbFe Thin Films", J. Appl. Phys., Vol. 55, (1984), p. 1760 .

45. R. B. van Dover, E. M. Gyorgy, R. P. Frankenthal, M. Hong, and D. J. Siconolfi, "Effect of Oxidation on the Magnetic Properties of Unprotected TbFe Thin Films", I. Appl. Phys., Vol. 59, (1986), p. 1291.

46. R. P. Frankenthal, D. J. Siconolfi, R. B. van Dover, and S. Nakahara, "Oxidation of an Amorphous Ironterbium Alloy", J. Electrochem. Soc., Vol. 134, $(1987)$, p. 235.

47. M. Hong, D. D. Bacon, R. B. van Dover, E. M. Gyorgy, J. F. Dillon Jr., and S. B. Albiston, "Aging Effects of Amorphous Tb-Transition Metal Films Prepared by Diode and Magnetron Sputtering", J. Appl. Phys., Vol. 57, (1985), p. 3900 . 
48. F. E. Luborsky, J. T. Furey, R. E. Skoda, and B. C. Wagner, "Stability of Amorphous Transition MetalRare Earth Films for Magneto-optic Recording", IEEE Trans. Magn., Vol. MAG-23, (1987), p. 156.

49. Imamura, N. S. Tanaka, F. Tanaka, and Y. Nagao, "Magneto-optical Recording on Amorphous Films", IEEE Trans. Magn., Vol. MAG-21, (1985), p. 1607.

50. T. Iijima and I. Hatakeyama, "Stability Properties of Indium Doped Amorphous Films for Magneto-optic Memory Applications", IEEE Trans. Magn," Vol. MAG$23,(1987)$, p. 2627.

51. Y. Watanabe, J. Tsuchiya, Y. Kobayashi, and $T$. Yoshitomi, "Magneto-optical Disk with Alumina Based oxide Layer", IEEE Trans. Magn., Vol. MAG-23, (1987), p. 2623.

52. M. Asano, M. Kobayashi, Y. Maeno, and K. Oishi, "Magneto-optical Recording Media with New Protective Films", IEEE Trans. Magn., Vol. MAG-23, (1987), p. 2620 .

53. E. E. Marinero, D. C. Miller, A. E. Bell, A. Gupta, R. N. Payne, and H. Notarys, "Oxidation Induced Magnetic and structural Changes in Magneto-optical Alloys", IEEE Trans. Magn., Vol. MAG-23, (1987), p. 2629 .

54. C. D. Wagner, W. M. Riggs, L. E. Davis, J. F. Moulder, and G. E. Meuilenberg, "Handbook of X-ray photoelectron spectroscopy", Perkin-Elmer Corporation, Eden Prairie, Minesota, (1979), p. 4.

55. J. B. Lumsden, "X-ray Photoelectron Spectroscopy", Metals Handbook, Vol. 10, (1986), p. 568 .

56. A. D. Romig, Jr., "Analytical Transmission Electron Microscopy", Metals Handbook, Vol. 10, (1986), p. 429.

57. J. W. Edington, "Practical Electron Microscopy in Materials Science", Van Nostrand Reinhold Co., $(1976)$, p. 10 .

58. A. E. Bell, "Antireflection Structures for Magnetooptic Recording", Topical Meeting on optical Data storage, Nevada, Technical Digest Ser. 10, (1987), p. 138 . 
59. F. Tanaka, Y. Nagao, and N. Imamura, "Dynamic Read Write Characteristics of Magneto-optical TbFeCo and DyFeCo Disk", IEEE Trans. Magn., Vol. MAG-20, (1984), p. 1033 .

60. J. C. Suits and K. Lee, "Giant Magneto-optical Kerr Effect in Euo", J. Appl. Phys., Vol. 42, (1971), p.3258. 